\title{
Index to volumes 1-20 (1950-1980)
}

\author{
Compiled by \\ HILKKA KOPONEN, LIISA SEPP ÄNEN and PENTTI ALANKO
}

\section{Editor's note}

This first cumulative Index of Karstenia covers all the papers so far published in the magazine. Karstenia, dedicated to the famous Finnish mycologist Petter Adolf Karsten (1834-1917), was first published at irregular intervals, between the years 1950-1976, and each issue was numbered as a separate volume (1-16). From volume 17 onwards, Karstenia has come out on an annual basis, each volume consisting of two issues (plus a supplement to vol. 18).

During this time Karstenia has established its position as an important medium for the studies of Finnish mycologists. Writers from abroad have contributed only occasionally, and mainly on topics in some way connected with Finland. The first papers in the magazine were mostly published in Finnish, but this practice was soon changed, as the interest abroad became evident. At present the studies in Karstenia are published in the main European languages: shorter articles intended for domestic use are published in Finnish and Swedish in another magazine of the Fin-

\section{Introduction}

The author index contains the authors in alphabetical order with the titles of their papers. When there are two or more authors, the co-authors are indexed with crossreferences.

The index of fungi contains all generic, infrageneric, specific and infraspecific names of fungi (incl. lichens, myxomycetes and bacteria) appearing in the journal. The occurrence of names in the lists of contents and the reference lists of the papers has been disregarded. Each scientific name is accompanied by nish Mycological Society, Sienilehti (earlier Sienitietoja-Svampnytt).

Unfortunately the exact dates of issue could not be traced for the early numbers of Karstenia. For the first eight volumes only the year of printing is known, for volumes 9-14 we know the month and year of printing. From vol. 15 onwards the exact date of publication has been recorded. These dates are found on the covers of the issues, and are not repeated here.

It is hoped that this Index will prove a great help to those using the issues of Karstenia. As the Editor of Karstenia, I wish to express my best thanks to Mrs. Hilkka Koponen, Lic.Phil., Miss Liisa Seppänen, M.Sc., and Mr. Pentti Alanko, Head Gardener (all from the Department of Botany, University of Helsinki), who have painstakingly performed the laborious task of compiling the material, mostly on a voluntary basis. I am also indebted to Prof. Teuvo Ahti and Dr. Harri Harmaja for reading the manuscript, giving valuable advice and helping with questions of orthography.

Tuomo Niemelä

the volume number(s) in bold-face type followed by the page number(s). The names of taxa above the rank of genus are not included, unless they are described as new or published as a new combination, and they are then followed by only one reference. Infrageneric names, i.e., subgenera (subg.), sections (sect.), and subsections (subsect.), are listed under the generic names, and infraspecific names, i.e., subspecies (ssp.), varieties (var.), forms (f.), and formae speciales (f.sp.), under the specific names. New names and combinations are printed in bold-face type. An attempt has been made to correct typographical and some orthographical errors in scientific names. 


\section{Portraits}

Kallio, Paavo - 18, frontispiece.

Karsten, Petter Adolf - 1: 5 .

Roivainen, Heikki - 17, frontispiece.

\section{Author index}

Aalto, Marjatta 1974: Amanita magnivolvata sp. nova (Agaricales). - 14: 93-96.

Ahti, Teuvo 1968: Micromycetes (Peronosporaceae, Erysiphales and Uredinales) new to the province of Kuusamo, N. E. Finland. - 8: 5-8.

Anon, 1950: Suomen Sieniseura r.y. Säännöt. Finlands Svampvänner r. f. Stadgar. - 1: 89-91.

- 1950: Ohjeita kirjoittajille. Anvisningar för författare. — 1: 91-92.

- 1953: Sientemme suomenkieliset nimet. Lakkisienet (Agaricales). Finska namn på hattsvampar. The Finnish names of Agaricales. - 2: 51-64.

Aro, Armo 1950: Sienikaupasta Suomessa (Summary: Mushroom trade in Finland). - 1: 85-87.

Balázs, Sándor \& Kovács, Andras 1978: Varianten in der Produktionstechnologie der Stropharia sp. in Ungarn. -18(suppl.): 53-55.

Bärlund, Ulla 1950: Laboratorieförsök beträffande rötsvamparnas inhördes konkurrensförmåga (Summary: Laboratory test concerning mutual competition of wood-destroying fungi). - 1: 60-72.

Dunaitsev, Vladimir 1978: Growing of champignons at 'Moskovski' Sovkhoz on synthetic substrate. 18(suppl.): $64-65$.

Gregory, Norma M. - See Watling \& Gregory 1977.

Gyllenberg, H. G. - See Hattula \& Gyllenberg 1969.

Gyurkó, Pal 1978: Einige Erfahrungen über Pleurotus ostreatus bei der Veredlungsarbeit. — 18(suppl.): 70-73.

Hæggström, Carl-Adam 1979: Geastrum nanum found in Finland. - 19: 19-21.

Hämet-Ahti, Leena - See Pyykkö \& Hämet-Ahti 1980.

Härkönen, Marja 1974: Über die finnischen Schleimpilzen. - 14: 54-81.

- 1977: Corticolous Myxomycetes in three different habitats in southern Finland. - 17: 19-32.

- 1977: Comatricha nannengae, a new species of Myxomycetes. - 17: 87-89.

- 1978: Lamproderma puncticulatum, a new species of Myxomycetes. - 18: 20-22.

- 1978: Comatricha ellae, nomen novum (Myxomycetes). - 18: 23.

- 1978: A new species of Myxomycetes, Physarum apiculosporum, described and cultivated. - 18: 24-26.

- 1979: Additions and corrections to the Finnish flora of Myxomycetes. - 19: 1-7.

- 1979: A check-list of Finnish Myxomycetes. - 19: 8-18.

Härkönen, Marja \& Koponen, Hilkka 1978: Myxomycetes developed on grain in moist chamber cultures. - 18: 58-62.

Harmaja, Harri 1969: A wider and more natural concept of the genus Gyromitra Fr. - 9: 9-12.

- 1969: A neglected species, Gyromitra ambigua (Karst.) Harmaja, n. comb., and G. infula s. str. in Fennoscandia. - 9: 13-19.

- 1969: Karstenella vernalis Harmaja, a new genus and species of Discomycetes from Finland. - 9: 20-22.

- 1969: On hygrophany of the basidiocarp in the genus Clitocybe Kummer. - 9: 51-53.

- 1969: The genus Clitocybe (Agaricales) in Fennoscandia. - 10: 5-168. 
- 1970: Type studies on Agaricales described as Clitocybe and Omphalina. - 11: 35-40.

- 1973: Amendments of the limits of the genera Gyromitra and Pseudorhizina, with the description of a new species, Gyromitra montana. - 13: 48-58.

- 1974: A revision of the generic limit between Clitocybe and Lepista. - 14: 82-92.

- 1974: Notes on the genus Helvella, including the merging of the genus Wynnella. - 14: 102-104.

- 1974: Flavoscypha, a new genus of the Pezizales for Otidea cantharella and O. phlebophora. - 14: 105-108.

- 1974: Two new families of the Pezizales: Karstenellaceae and Pseudorhizinaceae. - 14: 109-112.

- 1974: Singerella n. gen., a separate genus for Clitocybe hydrogramma. - 14: 113-115.

- 1974: Tarzetta pusilla n. sp. and T. spurcata (Pers.) n. comb. from Finland. - 14: 116-120.

- 1974: Notes on two agarics described in North America: 1. Rhodocybe smithii nom. nov., a new name for Clitocybe piperata A. H. Smith. 2. Cystoderma adnatifolium (Peck) n. comb., a species new for Europe. 14: $121-122$.

- 1974: Observations on the presence of cyanophilic perispore in the spores of the Pezizales. - 14: 123-125.

- 1974: Pseudoclitocybe atra (Vel.) n. comb. - 14: 126-128.

- 1974: Three new taxa of Lepista: L. fasciculata n. sp., L. singeri n. sp. and Lepista subgenus Laevispora $\mathrm{n}$. subg. - 14: 129-132.

- 1974: The generic limit between Otidea and Tarzetta (Pustularia auct.). - 14: 138-142.

- 1976: A further revision of the generic limit between Lepista and Clitocybe. - 15: 13-15.

- 1976: Type studies in Clitocybe. - 15: 16-18.

- 1976: Two new species of agarics from northern Fennoscandia: Clitocybe montana and Lactarius lapponicus. $-15:$ 19-22.

- 1976: The walls of the spores and basidia of Tricholoma found to be cyanophilic. - 15: 23-24.

- 1976: Hygrophorus pacificus (new for Europe) and H. hyacinthus (new for northern Europe) found in Kuusamo, northern Finland. - 15: 25-28.

- 1976: New species and combinations in the genera Gyromitra, Helvella and Otidea. - 15: 29-32.

- 1976: Paradiscina Benedix - synonym of Gyromitra Fr. - 15: 33-35.

- 1976: Another poisonous species discovered in the genus Gyromitra: G. ambigua. - 15: 36-37.

- 1976: Scanning electron microscopy of the spores of Gyromitra subg. Gyromitra and subg. Discina (Pezizales). -16 : 6-9.

- 1977: A note on Helvella solitaria (syn. H. queletii) and H. confusa n. sp. - 17: 40-44.

- 1977: A revision of the Helvella acetabulum group (Pezizales) in Fennoscandia. - 17: 45-58.

- 1977: Leucoscypha ovilla n. comb., a species new to Europe, found in northern Finland. - 17: 73-76.

- 1978: Suillus lapponicus, a new bolete species from northern Finland. - 18: 27-28.

- 1978: New species and combinations in the pale-spored Agaricales. - 18: 29-30.

- 1978: The division of the genus Lepista. - 18: 49-54.

- 1978: Phaeomarasmius confragosus - an agaric to be transferred to Tubaria. - 18: 55-56.

- 1978: New species and combinations in Helvella and Gyromitra. - 18: 57.

- 1979: Type studies in Clitocybe 3. - 19: 22-24.

- 1979: Studies in the genus Cystoderma. - 19: 25-29.

- 1979: Studies on cupulate species of Helvella. - 19: 33-45.

- 1979: Notes on Gyromitra esculenta coll. and G. recurva, a noteworthy species of North America. - 19: 46-49.

- 1979: Type studies in Clitocybe 4. - 19: 50-51.

- 1979: Mycena picta n. comb., an agaric new to Finland. - 19: 52-53.

Härö, S. - See Pätiälä \& Härö 1950.

Hattula, Marja Liisa \& Gyllenberg, H. G. 1969: Adaptability to submerged culture and amino acid contents of certain fleshy fungi common in Finland. - 9: 39-45.

- - 1969: Protein and fat composition and vitamin content of Boletus (Suillus) luteus mycelium produced in submerged culture. - 9: 46-50.

Heikkilä, Heli - See Kallio \& Heikkilä 1963, 1978.

Hietavuo, Seppo 1969: Boletus queletii Schulzer in Parainen, SW-Finland. - 9: 35-36.

Hintikka, Eeva-Liisa 1978: Toxicity of mushroom samples in cell culture system. - 18(suppl.): 40-42.

Hintikka, T. J. 1950: P. A. Karstenin elämäntyöstä. - 1: 5-14.

Hintikka, Veikko 1958: Über die finnischen Arten und Varietäten der Gattung Xeromphalina Kühner \& Maire. - 4: 5-9.

— 1961: Zur Ökologie einiger an Brandplätzen vorkommender Blätterpilzen. - 5: 100-106.

- 1961: Das verhalten einigen Mycena-Arten zum pH sowie deren Einfluss auf die Azidität der Humusschicht der Wälder. - 5: 107-121. 
- 1963: Studies in the genus Mycena in Finland. - 6-7: 77-87.

- 1963: Notes on Finnish Myxomycetes. - 6-7: 110.

- 1969: Acetic acid tolerance in wood- and litter-decomposing Hymenomycetes. - 10: 177-183.

- 1970: Stimulation of spore germination of wood-decomposing Hymenomycetes by carbon dioxide. - 11: 23-27.

- 1970: Selective effect of terpenes on wood-decomposing Hymenomycetes. - 11: 28-32.

- 1970: First record of Pycnoporellus albo-luteus in NW-Europe. - 11: 33-34.

- 1971: Tolerance of some wood-decomposing basidiomycetes to aromatic compounds related to lignin degradation. - 12: 46-52.

- 1971: Mucor oblongisporus as a psychrophilic secondary sugar fungus. - 12: 59-65.

- 1973: Passive entry of fungus spores into wood. - 13: 5-8.

- 1973: A note on the polarity of Armillariella mellea. - 13: 32-39.

- 1973: The role of carbon monoxide in the ecology of wood-decomposing Hymenomycetes: - 13: 44-47:

- 1974: Some type of mycorrhizae in the humus layer of conifer forests in Finland. - 14: 9-11.

- 1974: Notes on the ecology of Armillariella mellea in Finland. - 14: 12-31.

- 1974: Ceratocystis ulmi in Finland. - 14: 32.

Hintikka, Veikko - See Korhonen \& Hintikka 1980.

Hintikka, Veikko - See Samson \& Hintikka 1974.

Hintikka, Veikko, Korhonen, Kari \& Näykki, Ossi 1979: Occurrence of calcium oxalate in relation to the activity of fungi in forest litter and humus. - 19: 58-64.

Jalkanen, Esko - See Jalkanen \& Jalkanen 1978.

Jalkanen, Risto \& Jalkanen, Esko 1978: Studies on the effects of soil surface treatments on crop of false morel (Gyromitra esculenta) in spruce forests. - 18(suppl.): 56-57.

Kahanpää, Asko 1976: Geotrichum candidum and pulmonary diseases. - 15: 5-12.

Kallio, Paavo 1953: Lakkisieniä Ruissalon luonnonsuojelualueelta kuivuussyksynä 1951 (Summary: Agaricales in Ruissalo conservation area during autumn 1951). - 2: 48-50.

- 1963: Zur Verbreitung einiger in Finnland südlichen Pilze, besonders in der südwestlichen Eichenzone. 6-7: $35-76$.

- 1978: Macromycological research in Finland. - 18(suppl.): 21.

Kallio, Paavo \& Heikkilä, Heli 1963: Some macromycetes from Yyteri sand dunes in SW-Finland. - 6-7: 111-112.

- 1978: The boletes in Finland 1. Genus Boletus. - 18: 1-19.

Kallio, Paavo \& Kankainen, Esteri 1968: Contributions to the macromycetes in the oak zone of Finland. - 8: 9-13.

Kallio, Tauno 1971: Deposition of airborne fungal diaspores on special agar plates in Finland 1967-1968. - 12: 36-45.

- 1973: Influence of ultraviolet radiation on the colony formation of Fomes annosus (Fr.) Cooke diaspores suspended in water. - 14: 5-8.

Kankainen, Esteri 1969: On the structure, ecology and distribution of the species of Mitrula s. lat. (Ascomycetes, Geoglossaceae). - 9: 23-34.

Kankainen, Esteri - See Kallio \& Kankainen 1968.

Kankainen, Esteri - See Ohenoja.

Karanko, Sirkka-Liisa - See Kreula et al. 1976.

Karanko, Sirkka-Liisa - See Kreula et al. 1978.

Kauppi, Kaija K. \& Simola, Liisa Kaarina 1971: The effect of several sugars on the growth of Cladosporium herbarum and Trichothecium roseum. - 12: 53-58.

Klawitter, Maria \& Lasota, Wanda 1978: Labordiagnostik der Pilzvergiftungen unter besonderer Berücksichtigung der Vergiftungen mit dem Knollenblätterpilz Amanita phalloides. - 18(suppl.): 38-39.

Koistinen, Riitta 1978: The commercial mushroom yield in northern Finland in 1976. - 18(suppl.): $108-111$.

Koivurinta, Juha 1978: On the storage of some fresh wild mushrooms. - 18(suppl.): 81-84.

Koivurinta, Juha \& Kuusinen, Reijo 1978: Editors' note. - 18(suppl.): cover.

Koponen, Hilkka \& Mäkelä, Kaiho 1976: Phyllachora graminis, P. silvatica, Epichloe typhina and Acrospermum graminum on grasses in Finland. - 15: 46-55.

Koponen, Hilkka - See Härkönen \& Koponen 1978.

Koponen, Hilkka - See Mäkelä \& Koponen 1976.

Korhonen, Kari 1978: Interfertility and clonal size in the Armillariella mellea complex. - 18: 31-42.

- 1980: The origin of clamped and clampless basidia in Armillariella ostoyae. - 20: 23-27. 
Korhonen, Kari - See Hintikka et al. 1979.

Korhonen, Kari \& Hintikka, Veikko 1980: Simple isolation and inoculation methods for fungal cultures. - 20: 19-22.

Kostadinov, Jordan S. \& Stefanov, Spas L. 1978: Erforschungen der chemischen Zusammensetzung des Kulturträuschlings (Stropharia rugoso-annulata Farlow ex Murr.). - 18(suppl.): 100-101.

Kovács, Andras - See Balázs \& Kovács 1978.

Kovács, Etelka 1978: Cytokinins in cultivated champingon. - 18(suppl.): 93-96.

Kovács, Etelka - See Törley \& Kovács 1978.

Kovács, Etelka \& Zukál, E. 1978: Mechanism of after-ripening of the cultivated champignon (Agaricus bisporus) during storage. - 18(suppl.): 77-80.

Kreula, Matti - See Roponen \& Kreula 1978.

Kreula, Matti, Saarivirta, Maija \& Karanko, Sirkka-Liisa 1976: On the composition of nutrients in wild and cultivated mushrooms. - 16: 10-14.

- - 1978: Contents of nutrients and dietary fibre in wild and cultivated mushrooms. -18 (suppl.): 43-45.

K(ujala), V. 1953: T. J. Hintikka. Tohtori T. J. Hintikan julkaisuja. — 2: 5-8.

Kujala, Viljo 1953: Ascocalyx abietis ja sen kuromamuodot (Referat: Ascocalyx abietis und ihre Kondieformen). $-2: 35-38$.

Kukkonen, Ilkka - See Roivainen 1977: 4-5.

Kurkela, Rakel 1978: Opening. - 18(suppl): 3-4.

Kurkela, Rakel - See Mäkinen et al. 1978.

Kurkela, Rakel \& Matikainen, Eila 1978: Flavour intensity of some edible fungi. - 18(suppl.): 35-37.

Kurkela, Timo 1970: Lachnellula willkommii, lehtikuusensyövän aiheuttaja, ja Lachnellula occidentalis lehtikuusella Suomessa (Abstract: Lachnellula willkommii, the cause of larch cancer, and Lachnellula occidentalis on larch in Finland). - 11: 41-45.

- 1974: Godronia multispora Groves (Helotiales) and its pathogenicity to Betula verrucosa Ehr. and B. pubescens Ehr. - 14: 33-45.

- 1974: The association of Eupropolella vaccinii (Rehm) v. Höhn. and winter mortality of some ericaceous plants. - 14: 97-101.

Kuusinen, Reijo - See Koivurinta \& Kuusinen 1978.

Kwiatkowska, Anna - See Woźniak et al. 1978.

Laine, Lalli 1968: Notes on the polypores (Polyporaceae) of Ahvenanmaa. - 8: 14-20.

Lasota, Wanda - See Klawitter \& Lasota 1978.

Mäkelä, Kaiho 1970: The genus Mastigosporium Riess in Finland. - 11: 5-22.

- 1971: Some graminicolous species of Helminthosporium in Finland. - 12: 5-35.

- 1972: Spermospora ciliata (Sprague) Deighton and Scolecotrichum graminis Fuckel on Finnish grasses. 13: $9-15$.

- 1972: Some aquatic Hyphomycetes on grasses in Finland, - 13: 16-22.

- 1972: Rhynchosporium species on Finnish grasses. - 13: 23-31.

- 1979: Wojnowicia graminis on Gramineae. - 19: 54-57.

Mäkelä, Kaiho - See Koponen \& Mäkelä 1976.

Mäkelä, Kaiho \& Koponen, Hilkka 1976: Ovularia pusilla, Hadrotrichum virescens, Deightoniella arundinacea and Discosia artocreas on grasses in Finland. - 15: 38-45.

- - 1976: Telimenella gangraena and Septogloeum oxysporum on grasses in Finland. - 15: 56-63.

Mäkinen, Seija, Kurkela, Rakel \& Parikka, Tuula 1978: On the thiamine content of some edible mushrooms. 18(suppl.): 29-32.

Mäkinen, Yrjö 1963: On the smuts of the genus Ustilago on Calamagrostis species in Finland. - 6-7: 88-94.

- 1963: Zur Kenntnis der Micromycetenflora Finnlands 1. Für Finnland neue oder seltene Rost- und Mehltaupilze. - 6-7: 95-101.

- 1963: On Finnish Micromycetes 2. Puccinia malvacearum Bert. in Finland. - 6-7: 102-104.

- 1963: Plectania protracta (Fr.) Gelin in Finland. - 6-7: 105-107.

- 1963: Mutinus caninus Huds. and Peziza acetabulum L. in Finland. - 6-7: 108-110.

Mäkinen, Yrjö \& Pohjola, Aira 1969: Three discomycetous genera new to Finland. - 9: 5-8.

Maliranta, Helvi - See Pekkarinen \& Maliranta 1978.

Malmström, Nicken 1950: Tre finländska svamprariteter, Marasmiopsis subannulata (Trog.) Henn., Geastrum floriformis Vitt, och Geastrum bryantii Berk. (Summary: Three mushroom rarities in Finland: Marasmiopsis subannulata (Trog.) Henn., Geastrum floriformis Vitt., and Geastrum bryantii Berk.). - 1: 77-83:

Matikainen, Eila - See Kurkela \& Matikainen 1978. 
Mikola, Peitsa 1953: An experiment on the invasion of mycorrhizal fungi into prairie soil. - 2: 33-34.

- 1955: Metsämaan kantasienien puhdasviljely. (Summary: Growing forest soil Basidiomycetes in pure culture). $-3: 5-16$.

- 1958: Physiological variation in Collybia butyracea (Bull.) Fr. - 4: 10-13.

- 1959: Mycorrhizal fungi of exotic forest plantations. - 10: 169-176.

Mildh, Uolevi 1978: The organization for collecting forest mushrooms in Finland. - 18(suppl.): $106-107$.

Minter, D. W. 1979: Pseudodiplodia cenangiicola sp. nov., a fungus on Cenangium ferruginosum. - 19: 30-31.

Näykki, Ossi - See Hintikka et al. 1979.

Niemelä, Tuomo 1977: On Fennoscandian polypores 5. Phellinus pomaceus. - 17: 77-86.

- 1978: On Fennoscandian polypores 6. Antrodia plicata n. sp. - 18: 43-48.

- 1980: On Fennoscandian polypores 7. The genus Pycnoporellus. - 20: 1-15.

Niemelä, Tuomo \& Uotila, Pertti 1977: Lignicolous macrofungi from Turkey and Iran. - 17: 33-39.

Nikonorow, Maksym 1978: Utilization of and research on fungi in Polad. - 18(suppl.): 5-8.

Nyman, Antti 1968: Mycofloristic findings in Halikko, SW Finland. - 8: 21-24.

Ohenoja, Esteri 1974: Metsäsienien määrän mittaamisesta (Abstract: On the quantitative study of fungi in forest and bogs). - 14: 46-53.

- 1978: Recearch on mushroom yield in Finland. - 18(suppl.): 124.

Ohtonen, Rauni 1978: Mineral elements in some wild mushrooms. - 18(suppl.): 97-99.

Parikka, Tuula - See Mäkinen et al. 1978.

Pätiälä, Risto \& Härö, S. 1950: Review of fungi on the skin on the basis of the 1948 material. - 1: 48-59.

Pätiälä, Risto \& Rautavaara, Toivo 1950: Isaria cretacea van Beyma isolated from human nail in Finland (Isaria cretacea van Beyma löydetty ihmiskynnestä Suomessa). - 1: 83-84.

Pekkarinen, Maija \& Maliranta, Helvi 1978: Preliminary study on consumption of mushrooms in Finland. 18(suppl.): 46-48.

Pohjola, Aira - See Mäkinen \& Pohjola 1969.

Pohjola, Kalevi - See Raudaskoski et al. 1976.

Pyykkö, Maire \& Hämet-Ahti, Leena 1980: Sclerotinia pirolae: sclerotial ontogeny and occurrence in Finland. - 20: 28-32.

Rantala, Maija, Salmi, Leena, Sarkapalo, Tiina \& Visala, Leena 1978: Utilization of mushrooms in Pirkanmaa. - 18(suppl.): 112-119.

Rantcheva, Tzvetana 1978: Mushroom growing in Bulgaria. - 18(suppl.): 9-10.

- 1978: Introducing of ready-made and spawned compost for amateur mushroom growing. - 18(suppl.): 66-69.

Raudaskoski, Marjatta, Pohjola, Kalevi \& Saarvanto, Irmeli 1976: Effect of temperature and light on the mycelial growth of Gyromitra esculenta in pure culture. - 16: 1-5.

Rauhala, Aarre 1953: Die Verbreitung von Puccinia komarowii Tranzsch. in Finnland. - 2: 43-46.

- 1953: Aecidium-ruostelöytöjä (Referat: Aecidium-Funde). - 2: 46.

- 1953: Über ausgiebige Perithecienbildung bei Erysiphe Martii Lév. auf Melilotus albus Med. und bei Erysiphe verbasci (Jacz.) Blumer auf Verbascum nigrum L. (Selostus: Erysiphe Martiin Lév. runsasta periteekioiden muodostusta Melilotus albuksella Med.). - 2: 46-47.

- 1953: Puccinia veratri Niessl in Ostfennoskandien (Selostus: Puccinia veratri Niessl Itä-Fennoskandiassa). - 2: 47 .

- 1958: Kotimaisia härmäsienilöytöjä sekä tähänastiset tiedot härmäsienilajien maakunnittaisesta levinneisyydestä maassamme - Mehltaupilzfunde aus Finnland mit Berücksichtigung der bisherigen Verbreitungsangaben - Erysiphaceae in Fennia inventae et distributio earum hucusque cognita. - 4: 14-26.

Rautavaara, Toivo 1950: Myrkyllisiä ja myrkyllisiksi luultuja sieniä (Poisonous fungi and fungi believed to be poisonous). - 1: 15-47.

- 1950: Kalkkitehtaan vaikutusta sienikasvillisuuteen? (Influence of the lime factory on the mushroom vegetation). - 1: 85 .

- 1950: Katsaus sienikauteen 1949. - 1: 87-88.

- 1953: Juuritryffeli (Scleroderma bovista Fr.) lentohiekassa Pohjois-Suomessa (Summary: Scleroderma bovista Fr. on sand dunes in North-Finland). - 2: 50.

Rautavaara, Toivo - See Pätiälä \& Rautavaara 1950.

Rautavaara, Toivo \& Röyskö, Kaarina 1968: Ruokasienien säilöntä kuivaamalla (Summary: Dehydration of edible fungi). - 8: 25-60.

Roivainen, Heikki 1977: Resultados micológicos de la expedición a Argentina y Chile en 1969-1970 (Abstract: Mycological results of the expedition to Argentina and Chile 1969-1970). - 17: 1-18. 
Roponen, Ilma \& Kreula, Matti 1978: On the mycelial growth of the lorel or false morel, Gyromitra esculenta (Pers.) Fr. - 18(suppl.): 58-63.

Röyskö, Kaarina - See Rautavaara \& Röyskö 1968.

Saarivirta, Maija - See Kreula et al. 1976.

Saarivirta, Maija - See Kreula et al. 1978.

Saarvanto, Irmeli - See Raudaskoski et al. 1976.

Salmi, Leena - See Rantala et al. 1978.

Samson, Robert A. \& Hintikka, Veikko 1974: Mycosylva reticulata, a new psychrophilic hyphomycete. - 14: 133-137.

Sarkapalo, Tiina - See Rantala et al. 1978.

Saśek, Václav 1978: Techniques and problems of the isolation of pure cultures of Basidiomycetes. 18(suppl.): 49-52.

Schulmann, Otto von 1955: Pilzstudien in Finnland. - 3: 17-68.

- 1958: Pilzstudien in Finnland 2. - 4: 27-62.

- 1961: Zur Kenntniss der Basidiomyceten Finnlands. - 5: 5-99.

- 1963: Zur Kenntniss der Basidiomyceten Finnlands. - 6-7: 5-34.

Simola, Liisa Kaarina - See Kauppi \& Simola 1971.

Sobkowska, Eugenia - See Woźniak \& Sobkowska 1978.

Sobkowska, Eugenia - See Woźniak et al. 1978.

Sobkowska, Eugenia \& Woźniak, Wanda 1978: Combined processing of Tricholoma equestre. - 18(suppl.): 87-92.

Stanék, Miloslav 1978: State of production, utilization and research of cultivated mushrooms and other edible fungi in Czechoslovakia. - 18(suppl.): 17-19.

- 1978: Micro-organisms and cultivated edible fungi. - 18(suppl.): 74-76.

- 1978: Irradiation of fresh mushroom fruit-bodies. - 18(suppl.): 85-86.

Stefanov, Spas L. - See Kostadinov \& Stefanov 1978.

Suolahti, O. 1950: Puun lahoamis- ja lahonsuojatutkimuksista (Über Untersuchungen betreffend Holzfäule und Holzschutz). - 1: 73-76.

Suominen, Juha 1973: On the occurrence of the fungus Lentinus lepideus Fr. on the railway sleepers in Finland. - 13: 40-43.

Suominen, Raili 1969: Russula farinipes Romell apud Britzelmayr in Finland. - 9: 37-38.

Torev, Atanas 1978: Industrial production of concentrated protein from edible mushroom mycelium as food for people. - 18(suppl.): 20.

Törley, Dezsö 1978: Protein patterns of edible fungi. - 18(suppl.): 33-34.

Törley, Dezsö \& Kovács, Etelka 1978: Review of chemical research work on edible fungi in Hungary. 18(suppl.): 11-16.

Törley, Dezsö \& Vadon-Györey, E. 1978: Lipid patterns of edible fungi. - 18(suppl.): 22-28.

Tuomikoski, Risto 1950: Paxillus filamentosus Fr. (P. leptopus Fr.) in Finnland gefunden. - 1: 76-77.

- 1953: Die Lactarius-Arten Finnlands. - 2: 9-25.

- 1953: Notes on Finnish Agaricales. - 2: 26-32.

- 1953: Boletinus asiaticus Sing. in Finnland gefunden. - 2: 42.

Ulvinen, Tauno 1980: Urocystis carcinodes discovered in Finland. - 20: 16-18.

Uotila, Pertti - See Niemelä \& Uotila 1977.

Vaartaja, Olli 1953: Havaintoja puiden Dasyscypha-lajeista (Summary: Notes on the Dasyscypha-species of trees). $-2: 39-42$.

Vadon-Györey, E. - See Törley \& Vadon-Györey 1978.

Visala, Leena - See Rantala et. al. 1978.

Watling, Roy \& Gregory, Norma M. 1977: Larger fungi from Turkey, Iran and neighbouring countries. - 17: 59-72.

Woźniak, Wanda - See Sobkowska \& Woźniak 1978.

Woźniak, Wanda \& Sobkowska, Eugenia 1978: Juice concentrates of edible mushrooms. - 18(suppl.): 102-105.

Woźniak, Wanda, Sobkowska, Eugenia \& Kwiatkowska, Anna 1978: Mushroom and mushroom-vegetable pastes from wild edible mushrooms. - 18(suppl.): 120-123.

Zukál, E. - See Kovács \& Zukál 1978. 


\section{Index of fungi}

A bortiporus borealis 8: 14; 10: 178, 182.

Acetabula aestivalis 14: 103 .

- calyx 17: 41; 19: 42 .

- murina 19: $33,43,44$.

- simplex 17: 42.

— sulcata $5: 96 ; 6-7: 34 ; 17: 44,46$.

- vulgaris 17: 44.

Achorion 1: 48, 55 .

- gallinae 1: 50, 53, 55 .

- gypseum 1: 50, 55

Acrospermum 15: 53.

- compressum 15: 52 .

- graminum 15: 46, 51-53.

- - var. decipiens 15: 53 .

- - var. graminum 15: 53 .

Aecidium 2: 46.

- barbareae 2: 46 .

- ligulariae 2: 46.

- magellanicum 17: 12, 13.

- negerianum 17: 12, 14.

- tubiforme 17: 8, 12, 15 .

- ushuwaiense 17: 9, 12.

Aeruginospora hiemalis 14: 89 .

Aethalium septicum 19: 10, 11 .

_ - var. violaceum 19: 10, 11

Agaricus 2: 62; 4: 60; 5: 36, 38; 9: 39, 46; 10: 7, 8, 50, 114, 116,$180 ; 14: 91 ; 16: 14 ; 17: 60 ; 18$ (suppl.): $5,10,18$, $53-55,59.93,114,116,117$.

- abruptibulbus 3: 65; 4: 49, 60, 62; 5: 37 .

- adirondackensis 14: 114 .

- adsentiens 10: 114.

- aeruginosus (-a) 2: 31, 32 .

- aestivalis 6-7: 14 .

- amethystinus 5: 38 .

- anisarius 19: 50 .

- annae 6-7: 14.

- arvensis $2: 52 ; 3: 21,27,31,35,46,56 ; 4: 42 ; 5: 37 ; 6-7: 14$; 9: 40-42; 18(suppl.): 25-27.

- - var. subexquisitus $3: 21 ; 5: 37$.

— augustus $2: 52 ; 3: 65 ; 4: 62 ; 5: 37,38 ; 6-7: 14$.

_ - var, albus $3: 65 ; 5: 38$.

- bernardii 6-7: 14

- bisporus $3: 27,35,46 ; 5: 36 ; 8: 31,33,34,47,48,57,59 ; 16:$ 3; 18(suppl.): 6, 11-13, 17, 24-27, 29-31, 33-37, 53, 67, 71, $74-77,81-83,88,93$.

- - f. avellanea 5: 36,37 .

- bitorquis 5: 37; 17: 60; 18(suppl.): 17, 18, 67, 74, 75 .

- brunneolus $3: 46 ; 5: 38$.

- callisteus 18(suppl.): 7 .

- campestris(-er) 2: 52; 3: 21, 35, 46, 4: 33, 49, 60; 5: 37; 6-7: 14; 9: 46; 16: 13; 18(suppl.): 6, 39, 93.

_- equestris $4: 33 ; 5: 37$.

_- squamulosus $3: 56 ; 5: 37$.

- candicans 10: 74, 75 .

- carnosior 19: 50.

- catinus 10: 63,64.

- cerussatus 10: 87.

- chionodermus 6-7: 14

- cinnabarinus 19: 27.
- clavipes 4: 12,59 .

- clitocyboides 14: 113, 115; 15: 16,17.

— comtulus 3: 56; 4: 49; 5: 38; 6-7: 14, 15.

- confragosus 18: 55 .

- conigenus 2: 29,30 .

- cretaceus $3: 21 ; 5: 37$.

- cuprobrunneus 6-7: 14.

- cyaneus 2: 31,32 .

- cyathiformis 14: 128

- dealbatus 10: 75, 76 .

- diatretus 10: 90; 15: 14.

- dicolor 10: 97.

- ditopus 10: 105.

— edulis $4: 33 ; 5: 37$.

- esculentus 2: 29 .

- excellens 5: 38 .

- fissuratus 5: 37 .

- fragrans 10: 93, 95; 15: 14: 18: 54.

- fulveolus $3: 46 ; 5: 38$.

- geotropus 10: 61 .

— gibbus 10: 64 .

- gilvus 14: 84.

- granulosus cinnabarinus 18: 30; 19: 27.

- haemorrhoidarius $3: 46,65 ; 5: 37$.

- helvelloides $3: 63 ; 5: 17$.

- hortensis 5: 36; 18(suppl.): 6 .

- - var. bisporus 3: 27; 5: 36 .

- hydrogrammus 10: 82; 14: 113, 114.

— infundibuliformis 10: 60, 61, 65 .

- ingratus 5: 37.

- inornatus 10: 55 .

- interveniens 10: 114.

— irinus 14: 132.

- jasonis 18: 29; 19: 25.

- langei $3: 46 ; 5: 37$.

- lepista 14: 82, 90, 91; 18: 49-52.

- leucotrichus $3: 65 ; 5: 37$.

- macrocarpus 5: 37; 6-7: 14.

- macrosporus 3: 27; 5: 38 .

- mediofuscus 5: 37.

- melleus 18: 31 .

- metachrous 10: 96, 97; 15: 14.

- nebularis 10: $5,12,50,52,56,57,116 ; 14: 91$.

— nivescens $4: 33 ; 5: 37$.

_- var.parkensis 6-7: 14.

- odorus 10: 84; 15: 15 .

- osecanus 6-7: 14.

- pachyphyllus 19: 23.

- perrarus $3: 21 ; 5: 37$.

- phalloides 8: 10 .

— phyllophilus 10: 86,$87 ; 15: 15$.

- pictus 19: 52.

- pithyophilus 10: 88,89 .

- politus 2: 32

- porphyrius 8: 10 .

- pruinosus 10: 77, 79 .

- puellula 10: 115 .

- purpurellus 5: 38 .

- rodmanii 17: 60

— rubellus 3: $35,46,56 ; 5: 38$.

— rusiophyllus $3: 35,46 ; 4: 33,49,60 ; 5: 38 ; 6-7: 14,15$.

- sanguinarius 5: 37.

- semotus 5: 38: 17: 60 .

- silvaticus 2: 52; 3: 21, 27, 35; 5: 37; 18(suppl.): 6, 25-27.

- - var. pallens 6-7: 14

— silvicolus $2: 52 ; 3: 21,27,35,46,56,65 ; 4: 49,60 ; 5: 37,38$.

- sinopicus 10: 69 . 
- sinuatus 8: 10

- squamulosus 10: 65.

- stramineus 5: 38 .

- suaveolens 10: 95.

- subfloccosus 6-7: 14.

- subperonatus 4: 33; 5: 37.

- subviscifer 10: 115 .

- svaveolens 18: 49, 54.

- tenacellus 2: 29, 30.

- terreii 18: 30; 19: 27, 28.

- thejogalus 15: 21 .

- undatus 17: 63 .

— urinascens $3: 52 ; 5: 38$

— vibecinus 10: 103-105.

— villaticus $3: 52 ; 5: 38 ; 6-7: 14$.

- xanthodermus $3: 65 ; 4: 62 ; 5: 37$.

Agrocybe 2: 61; 5: 64; 6-7: 24; $14: 87$.

- aegerita 18(suppl.): 18 .

— arenicola 17: 61.

- arvalis $5: 64$.

— dura 3: 59; 5: 64; 14: 87.

- erebia 5: 64 .

— ombrophila 5: 64; 6-7: 24.

— paludosa 3: 59; 4: 55; 5: 64 .

— pediades 3: 59: 5: 64; 6-7: 24 .

— praecox 2: 52; 3: 22, 31, 40, 49, 59; 4: 37, 55, 61; 5: 64, 72; 10: 180.

— semiorbicularis $3: 59 ; 5: 64 ; 6-7: 24 ; 17: 60$.

- sphaleromorpha 3: 59; 4: 37, 55; 5: 64, 67; 6-7: 26.

— tabacina 4: 37, 55; 5: 64 .

- vervacti $3: 40 ; 5: 64$.

Albatrellus ovinus 16: 10, 12, 13; 18(suppl.): 29-31, 41-43, $107,115,117$.

Albugo candida 8: $6 ; 17: 3$.

- tragopogonis 17: 3 .

Aleuria 9: 6; 14: 125

— aurantia 3: 30, 53, 62; 4: 59; 5: 96; 9: 6,7; 18(suppl.): 116 , 117.

Aleurisma 1: 56, 58.

Alnicola 6-7: 18, 21.

- alnetorum 5: 48 .

— amarescens $3: 65 ; 5: 47$.

— badia 4: $51 ; 5: 47$.

— bohemica $3: 37 ; 4: 51 ; 5: 47$.

- conspersa $3: 65 ; 4: 35 ; 5: 47$.

- escharoides $2: 52 ; 3: 65 ; 4: 35,51 ; 5: 47$.

- luteolofibrillosa $3: 65 ; 4: 51 ; 5: 47 ; 6-7: 18$.

- macrospora f. tetraspora 4: 51; 5: 48.

— melinoides $3: 65 ; 4: 35 ; 5: 47$.

— phaea $4: 35,51 ; 5: 47$.

- pseudoamarescens 4: 51; 5: 47; 6-7: 18

— scolecina $3: 21 ; 4: 51 ; 5: 47$.

_ $-\mathrm{f}$. gracillima $4: 51 ; 5: 48$.

— scorpioides $3: 37 ; 5: 47$.

— subconspersa 3: 65; 4: 35, 51; 5: 47.

— submelinoides $3: 57,65 ; 4: 35,59 ; 5: 48 ; 6-7: 18$.

- umbrina 5: 47, 48 .

Alternaria 1: 54, 56, 58, 59; 12: 42.

Alysidium 14: 135.

Amanita 1: 18, 22, 26, 39, 40; 3: 6, 9, 10, 16; 4: 60; 10: 170, 171; 14: $93,95,96 ; 18$ (suppl.): $39,50,51,116$.

- ampla 5: 36.

— argentea 14: 93-95.

- bulbosa 8: 10 .

- caesarea 14: 96

— citrina $1: 26,40 ; 2: 52 ; 5: 36 ; 6-7: 50,60,69-72 ; 8: 11$; 18(suppl.): 41 .
— crocea $3: 56 ; 4: 33,49$

— excelsa $1: 28 ; 5: 36 ; 6-7: 50$.

- fulva 6-7: 70 .

- hyperborea 19: 42 .

- inaurata 5: 35; 6-7: 14

— junquillea 6-7: $51 ; 8: 11$.

- lividopallescens 6-7: 49; 14: 93, 94, 96

- magnivolvata 14: 93-96.

- mappa 1: 25, 26, 40; $2: 52 ; 3: 7,8,21,27,46 ; 5: 36 ; 6-7$ : 50; 8: 11 .

- - var. alba 5: 36; 6-7: 14

- - var. phalloides 8: 10.

- muscaria 1: 24-27, 36, 40,41; 2: 52; 3: 7, 10, 21, 27, 31, 35, 46,$56 ; 4: 33,49,60 ; 6-7: 37,50,70 ; 10: 170,172 ; 16: 13$; 18(suppl.): 40-42.

_ - f. (var.) aureola $3: 21,35 ; 4: 49 ; 5: 35$.

- nivalis 5: 35 .

- pantherina 1: 20,24, 25, 27, 28, 36, 40,41;2:52;3:7,8, 31,$46 ; 5: 36 ; 6-7: 46,51,56,60,65,69,70-73 ; 8: 10 ; 18: 3$.

— phalloides 1: 20-28, 36, 37, 40, 46; 2: 52; 6-7: 69; 8: 10,11; 10: 171,$172 ; 18$ (suppl.) 7, 38, 39, 41 .

— - var. alba 8: 10 .

- porphyria $1: 25,27,36,40 ; 2: 52 ; 3: 7,10,21,27,31,35$, 46,$56 ; 4: 33,49,60 ; 5: 36 ; 8: 10$.

- pseudorubescens 1: 27, 41 .

- regalis $2: 52 ; 3: 21,27,31,35,46,56 ; 4: 49,60 ; 5: 36$.

- rubescens 1: 18, 25, 27, 28, 38, 40, 41; 2: 52; 3: 7, 8, 21, 27, $31,35,46,56 ; 4: 33,49,60 ; 5: 36 ; 6-7: 60,69,70 ; 8: 47$, 48,$59 ; 12: 55 ; 18$ (suppl.): 25-27, 116, 117.

- spissa 1: 27, 28, 40, 41; 2: 52; 5: 36; 6-7: 50, 69-70, 72; 8: 11.

- - var. valida 5: 36 .

— strangulata $3: 35,46 ; 4: 42 ; 5: 35 ; 6-7: 50$.

- umbrina 1: $25,27,28,36,40,41 ; 5: 36$.

- umbrinolutea $4: 49 ; 5: 35$

— vaginata $2: 52 ; 3: 7,10,21,27,46 ; 4: 33,49,60 ; 5: 35 ; 6-7$ : 49, 50, 70; 8: 47,48; 14: 94, 96; 17: 60; 18(suppl.): 41, 116,117

— - var. alba $3: 21,35,46,56 ; 4: 49 ; 5: 35 ; 6-7: 14$.

— - var. badia $3: 21,31,35,46,56 ; 4: 33,49 ; 5: 35$.

— - var. crocea $3: 52 ; 5: 35 ; 6-7: 14$.

_ - var. fulva $3: 21,31,35,46,56 ; 4: 33 ; 5: 35$.

_ - var. lividopallescens 6-7: 46, 49.

_ - var. lutescens $3: 21,35 ; 4: 33 ; 5: 35 ; 6-7: 14$.

- - var. major 6-7: 49 .

— - var. plumbea $3: 21,31,35,46 ; 5: 35 ; 6-7: 14$

— valens 17: 59, 60 .

— valida 1: 28; 5: 36 .

- verna 1: 23, 24.

- virosa 1: 15, 20, 23-25, 27, 28, 36, 37, 40, 41, 46; 2: 52; 3: 7, $10,27,31,35,46,56 ; 5: 36$.

Amanitopsis strangulata 6-7: 49, 50.

— vaginata 1: 27,$40 ; 2: 52 ; 8: 59$.

Amaurochaete atra 19: 10, 13 .

- fuliginosa 6-7: 110; 14: 69; 19: 10 .

Amylocystis lapponica 20: 6, 7 .

Amyloporia 13: 6 .

— calcea 8: 14 .

- xantha 8: 14; $20: 7$.

Anellaria 2: 61, 63.

- semiglobata 4: 38 .

- semiovata 2: 52; 5: 70 .

— separata $2: 52 ; 3: 22,28,40,49 ; 4: 38,56 ; 5: 70 ; 6-7: 26$.

- - var. minor 4: 38; $5: 70$.

- - var. vernalis 5: 70 .

- sepulchralis 17: 62 .

Angioridium sinuosum 19: 10, 12. 
Anisomyces odoratus 8: 15; 10: 178, 182.

Anthopeziza 6-7: 105.

- protracta 6-7: 105.

- winteri 6-7: 105.

Anthracoidea 17: 4, 5.

- ortegae 17: 1, 4, 5.

- pulicaris 17: 4 .

- rupestris 17: 4.

Anthurus australiensis 6-7: 109

Antrodia 18: 43, 46, 48.

- albida 18: 46, 47.

- heteromorpha 18: 46.

- juniperina 18: 46.

- malicola 18: $46,47$.

- mollis 8: 15 .

_ plicata 18: $43,44,46,47$.

- salicina 18: $43,48$.

- serpens 18: 47.

- sinuosa 18: 46.

- variiformis 18: 46.

Aphanomyces astaci 12: 55.

Aporpium 13: 6 .

- caryae 17: 38 .

— semisupinum 8: 15; 10: 181; 12: 48, 49, 63.

- vulgare 8: 15.

Apostemidium fiscella 3: 62; 5: 97.

Araispora 12: 55.

Arcyodes incarnata 14: 78; 19: 10, 12-14.

Arcyria 14: 76-79; 17: 31.

— adnata 19: 10.

- cinerea 14: 76; 17: 19, 22-24, 29-31; 18: 60; 19: 10 .

- denudata 14: 76, 79; 19: 10.

-- ferruginea 14: 76, 79; 19: 10 .

— incarnata 6-7: 110; 14: 77, 80; 17: 22; 19: 10.

- nutans 6-7: 110; 14: 77, 80; 19: 10, 14 .

- obvelata 19: 10, 14 .

- oerstedtii 14: 78; 19: 10.

— pomiformis 14: 76; 17: 19, 22-24, 29-31; 18: 60; 19: 10.

- punicea 19: 10 .

- stipata 14: 77; 19: 10, 14.

Armillaria 1: 28, 41; 2: 52; 18: 31, 38, 41 .

- bulbiger 1: 88 .

- goliath 6-7: 38 .

- jasonis 19: 25 .

- mellea 1: 18,28, 88; 2: 49, 50, 3: 7; 4: 46; 9: 40; 10: 181; 14: 12; 17: 67; 18: 31; 18(suppl.): 5; 20: 23 .

Armillariella 2: $52 ; 10: 10 ; 18: 31,32,34-41 ; 18$ (suppl.): 82; 20: $21,23-26$

- bulbosa 18: 31-35, 37, 41 .

- elegans 14: 19, 24.

- limonea 18: 33, 35.

- mellea 2: 53; 3: 20, 26, 30, 34, 45, 55; 4: 31; 5: 18; 6-7: 37, $42 ; 8: 37,46-48,59 ; 9: 40-44 ; 10: 10 ; 11: 26,31 ; 12: 48,49$, 63; 13: 5-7, 32-34, 36-38; 14: 12-29; 16: 10, 12, 13; 18: $31-35,37,38,40,41$; 18(suppl.): 6-7, 26, 27, 41-43, 49, $81-83,102,104,115,117 ; 20: 19,23,26$.

- novaezelandiae 18: 33,35 .

- ostoyae 18: 38; 20: 21, 23-26.

Arthrinium ushuwaiense 17: 14.

Ascobolus 10: 180.

- immersus 12: 55.

Ascocalyx $2: 37,38$

— abietis 2: $35,36,38$.

Ascocorticium 9: 22 .

Ascosorus 9: 22.

Aspergillus 1: 56, 58, 59; 18(suppl.): 51, 86.

— fumigatus 15: 10; 18(suppl.): 86.

- nidulans 12: 55.
- niger 1: 58; 11: 25.

Aspropaxillus 2: 54.

- giganteus 2: 53; 3: 20.

Asterophora 2: 60 .

- lycoperdoides $2: 53 ; 3: 20,44 ; 4: 45 ; 5: 11 ; 6-7: 7 ; 10: 26$.

- paracitica 3: 20; 5: 11

Aurantioporellus 20: 1 .

- alboluteus 20: 1, 2 .

Aureoboletus cramesinus 18: 17.

Auxarthron 15: 10.

Bacidia chlorococca 17: 19, 26, 30.

Bacillus extorquens 19: 58.

- macerans 18(suppl.): 75.

- mesentericus 18(suppl.): 75.

Badhamia 14: 58; 17: 31 .

— capsulifera 14: 56; 19: 10, 14 .

- decipiens 14: 56; 19: 10, 12 .

— foliicola 14: 56; 19: 1, 10.

- hyalina 19: 10.

- lilacina 19: 1, 2, 10, 14.

- ovispora 18: 25.

— utricularis 14: 56; 19: 10, 12-14.

— - var. melaleuca 19: 10, 14.

Baeospora 2: 54.

- myosura $2: 30,53 ; 3: 27,34,45,64 ; 4: 32,48 ; 5: 29 ; 6-7$ : 11 .

Bankera 6-7: 30.

— fragilis 6-7: 30 .

- fuligineoalba 6-7: 30 .

— violascens 6-7: 30 .

Beauveria 1: 84

Belodinium pruinosum 6-7: 34 .

Bipolaris 12: 5 .

- sorokiniana 12: 26.

Bjerkandera adusta 8: 15; 10: 181 .

Bolbitius 1: 31

- boltonii 3: 28, 40 .

— fragilis $3: 48,59 ; 4: 37$.

- titubans 3: 40, 49, 67.

— vitellinus $2: 53 ; 3: 22,40,48,59 ; 4: 37,55 ; 5: 64 ; 6-7: 24$.

- - f. boltonii 5: 64 .

- - var. (f.) fragilis 4: 55; 5: 64; 6-7: 24.

_ - f. maximus (-a) 4: 37; 5: 64; 6-7: 24 .

— - f. titubans 4: 37; 5: 64 .

Boletinus 2: 42.

- asiaticus 2: 42; 6-7: 6; 10: 169.

- cavipes 2: 42; 6-7: 169

Boletus 1: 7, 10, 35, 36, 45, 46; 2: 27, 52, 53, 56, 58, 63, 64; 3: $6,10,16 ; 6-7: 56,60 ; 17: 77 ; 18: 1 ; 18$ (suppl.): 5-7, 29, 31, 46-49, 108.

- aestivalis 6-7: 60; 18: 8, 17 .

— albidus 18: 3, 14 .

- appendiculatus 18: 17 .

- - ssp. pallescens 18: 17.

- atkinsonii 6-7: 55.

- aurantiacus 16: 12

- aureus 18: 6 .

— badius 3: 7, 10, 11; 18: 3, 4 .

- barlae 9: 36

- bovinus $3: 7,9,10$.

- calopus 6-7: 60,$70 ; 18: 5$.

- carpini 2: 26; 6-7: 59.

- chrysenteron 18: 3, 5, 6, 9, 13-16.

- crocipodius 6-7: 58 .

- cramesinus 18: 17 .

- cyanescens 1: 25.

- duriusculus 2: 28 :

- edulis $1: 88 ; 2: 53 ; 3: 7,10,11,19,25,30,33,43,54 ; 4: 29$, 
45,$60 ; 5: 8 ; 6-7: 37,55-57,60,70 ; 8: 37,46,48,49,51,56$, 58-60; 9: 39, 40-42, 46; 10: 171, 172; 16: 10-13; 18: 1, 6, 7, 17; 18(suppl.): 5-7, 17, 29-31, 35-37, 39, 41-44, 107, 108, $115,117$.

- - f. arcticus 18: 6, 7.

- $\mathrm{f}$. aurantioruber 18: 6,7 .

- $\mathrm{f}$. betulicola 18: 6 .

- f. citrinus 18: 6,7 .

- - ssp. edulis 6-7: 70; 18: 6, 7 .

- - f. arcticus 18: 1,6 .

- - $\mathrm{f}$. aurantioruber 18: 1,6 .

- $-\mathrm{f}$. citrinus 18: 1,6 .

- $\mathrm{f}$. laevipes 18: 6 .

- $\mathrm{f}$. piceicola 18: 6 .

- - ssp. (var.) pinicola 2: 27; 6-7: 6, 56; 18:6, 7 .

- - var. fuscoruber 18: 7

- - f. quercicola 18: 6 .

- - ssp. reticulatus $6-7: 53,55,56,60,65,68,70,73 ; 8: 12$; 18: 6-9, 17

— elegans 2: 42; 3: 7, 10, 11; 10:169.

- erythropus $1: 35,45 ; 5: 8 ; 6-7: 6,56,57,60,70,73 ; 8: 12$; 9: $35 ; 17: 61 ; 18: 3,8,9,13-15$.

- fechtneri 6-7: 60; 18: $3,4,17$.

— felleus $1: 25,35,40,45 ; 3: 7,10 ; 6-7: 70$.

- ferrugineus 18: 16 .

- flavidus 1: 25 .

- hemichrysus 18: 9 .

- holopus 2: 26.

- impolitus 18: 1, 10 .

- junquilleus 17: 61 .

- lanatus 18: 1, 5, 15, 16 .

- leguei 18: 16.

- leucophaeus 2: 26.

- lignicola 18: 10

- lupinus 1: 25. 35, 45 .

- luridus $1: 25,35,45 ; 3: 19 ; 5: 8 ; 6-7: 53,56,57,73 ; 8: 12 ; 9$ : $35 ; 18: 7-9,11,15$.

- luteoporus 6-7: 58.

- luteoscaber 6-7: 58

- luteus 3: 7, 10; 8: 37, 46, 48, 58; 9: 44, 46-49.

- miniatoporus $1: 25,35,45,5: 8 ; 6-7: 6,53,57,70 ; 9: 35$; 18: 8 .

- nigrescens 2: 26; 6-7: 58 .

- pachypus 6-7: 60, 70; 18: 5 .

— parasiticus 18: 17 .

— pinicola 18: 7

- pinophilus 18: 7 .

— piperatus 1: $25,35,40,45 ; 3: 7,10 ; 18: 1,12 ; 18$ (suppl.): $41,116,117$.

- - var. amarellus 18: 12

- pomaceus 17: 77, 80 .

- porosporus 18: $13,15,16$

- pseudoscaber $2: 26,27 ; 6-7: 59$.

- pulverulentus 6-7: 70; 18: 17.

- purpureus 18: 17 .

— queletii 9: 35,$36 ; 17: 61 ; 18: 3,8,9,13,14$.

- - var. lateritius 9: 35

- radicans 18: 3.

- reticulatus $6-7: 55,56 ; 18: 7,8$.

- rhodoxanthus 9: 35; 18: 17.

- roseofractus 6-7: 59 .

— rubellus 9: $36 ; 18: 14$.

— rubinellus 16: 3 .

- rufescens $2: 27: 16: 12$

— rufus $1: 87,88 ; 2: 26 ; 3: 7,10$.

- rugosus 2: 26

- sanguineus $3: 7,10,54 ; 9: 36$.

- satanas 1: 16, 23-25, 35, 39, 45; 6-7: 60, 72; 9: 35, 36; 18:
15.

— scaber $1: 87,88 ; 2: 26 ; 3: 7,10 ; 8: 46,48,58 ; 18$ (suppl.): 29.

- spadiceus 18: $1,15,16$

- subtomentosus 3: 7, 10,11;18: 1, 5, 15-17; 18(suppl.): 41 .

- - var. luteolus 18: 15 .

- suillus 16: 12

- sulphureus 18: 9 .

- tesselatus 6-7: 58 .

— testaceoscaber 8: 37.

- truncatus 18: 13 .

— variegatus 1: 85; 3: 7, 9-11; 8: 46, 58.

- variipes 6-7: 55 .

— versicolor 3: 54; $4: 29 ; 9: 36 ; 18: 14,15$.

— versipellis $2: 26,27 ; 8: 39,46,48,58 ; 9: 40,41$.

Bethrodiscus 2: 35-38.

- abietis 2: 37 .

- pinicola 2: 35-38.

Botryotinia squamosa $16: 3$.

Botrytis cinerea 17: 14; 18(suppl.): 51 .

Bovista 17: 70; 18(suppl.): 116, 117.

— nigrescens 3: 23, 29, 41, 50, 61; 4: 40, 58; 5: 94.

- paludosa 18: 30 .

- pila 17: 70 .

- plumbea $3: 23,29,41,50,61 ; 4: 40,60 ; 5: 94 ; 17: 70$.

- polymorpha 17: 60,70 .

Brachysporium flexuosum 12: 6 .

Brefeldia maxima 14: 69; 19: 1 .

Bremia lactucae 8: 6 .

Brunchorstia 2: 36.

Buglossoporus pulvinus 17: 33, 36 .

Bulgaria inquinans 8: 9.

Byssonectria 14: 125 .

Calocera cornea 3: 42; 5: 82; 6-7: 32.

— flammea $3: 23 ; 4: 58 ; 5: 82$.

- - var. furcata $5: 82$.

- furcata 3: 61 .

- palmata 3: 53, 50; 4: 43; 5: 82 .

— viscosa $3: 7,8,23,29,42,50,61 ; 5: 82$.

- - f. dilata 5: 82 .

- - f. furcata 3: 50 .

Calocybe 5: 11; 6-7: 7; 15: 16, 18; 18: 50; 19: 24, 50, 51.

- aromatica 15: 16 .

- cerina 6-7: 7 .

- chrysenteron 6-7: 7 .

- gambosa 15: 16, 18.

— georgii 3: 20, 44; 4: 60; 5: 12; 6-7: 54, 69; 18(suppl.): 93.

— ionides $4: 29 ; 5: 12$.

- juncicola 6-7: 7 .

- onychina $4: 45 ; 5: 12$.

- persicolor $3: 44 ; 5: 12 ; 6-7: 7,10: 61$.

- pusilla 19: 50,51 .

Calodon 4: 60; 5: 87, 88; 6-7: 30, 31; 14: 97

- aurantiacum 3: 24, 29, 42, 51; 5: 87.

— caeruleum 3: 24; $5: 87$.

- compactum 3: 29; 5: 87 .

- cyathiforme $3: 24,29,32,42,51,61 ; 5: 88$.

— ferrugineum 3: 24, 29, 42, 51; 5: 87; 14: 98.

- geogenium 3: 51; 5: 87 .

- graveolens $3: 51 ; 5: 88$.

- - var. ramosum 5: 88 .

- melaleucum 3: 51, 53; 5: 88.

— nigrum 3: 51, 53; 5: 88; 6-7: 31 .

- scrobiculatum 3: 29, 42, 51; 5: 88 .

- spadiceum 5: 88 .

— suaveolens 3: 24, 29, 51; 5: 87

— sulphureum 3: 51, 61; 5: 87.

- zonatum 3: 51; $4: 40 ; 5: 88$. 
- - var. queletii 5: 88 .

Calomyxa metallica 17: 22; 19: 10, 12, 14.

Caloscypha 14: 124.

Calvatia 6-7: 32; 18(suppl.): 116, 117.

— caelata 3: 23, 29, 41, 50; 4: 58; 5: 94

- cyathiformis 17: 70 .

— gigantea 5: 94.

- maxima 5: 94.

— saccata 3: 50, 67; 4: 43, 58; 5: 94.

- - var. pistilliformis 4: 28; 5: 94; 6-7: 32 .

Camarophyllus 1: 32,$44 ; 2: 53 ; 3: 10 ; 5: 9,10$.

- atropunctus 5: 18.

- bicolor 6-7: 71.

- cinereus 6-7: 6 .

- colemannianus 5: 10 .

- leporinus 5: 9; 6-7: 6 .

— niveus $2: 57 ; 3: 25,43,62 ; 5: 9$.

- ovinus (-a) 3: 33 .

- pratensis $2: 57 ; 3: 19,20,33,43,54,61 ; 4: 45 ; 5: 9,81 ; 6-7$ : 6; 18(suppl.): 116, 117 .

- russocoriaceus $3: 62 ; 5: 9$.

- streptopus 3: 43 .

- subradiatus 5: 9, 10.

— virgineus $2: 57 ; 3: 25,33,62 ; 5: 9$.

- - var. fuscescens 5: 9 .

Candida 1: 56; 9: 46

— albicans 1: 56, 57, 59; 15: 9, 11 .

- guillermondii 1: 56, 57.

- krusei 1: 56.

- tropicalis 1: $56,57,59$.

Cantharellula 5: 23; 6-7: $8 ; 10: 9,10$.

- concava 6-7: 10 .

- cyathiformis $2: 53 ; 3: 25,26,34,52 ; 4: 47 ; 5: 12,15,20$; 6-7: 10 .

—obbata $3: 52 ; 4: 42,47 ; 5: 20 ; 6-7: 10$.

- umbonata $2: 53 ; 3: 26,34,45,55,63 ; 4: 31,46 ; 5: 20 ; 10$ : $10,11,32 ; 14: 50,51$.

Cantharellus 1: 35,$45 ; 3: 8,16,61 ; 5: 23,81 ; 8: 26 ; 10: 171$; 18(suppl.): 46-48, 82 .

— aurantiacus 1: 19, 25, 35; 2: 49; 9:40.

— albidus 3: 50, 67 .

- cibarius 1: 17; 3: 7, 8, 23, 29, 32, 41, 50,61; 4: 40, 42, 58, $60 ; 5: 81 ; 8: 46,48,56,58 ; 9: 39,41-44 ; 16: 11-13 ; 17: 69$; 18(suppl.): $5-7,17,29-31,35-37,41-45,81-83,102-105$, $107,114,115,117,120-123$.

_ - var. bicolor 6-7: 28 .

- - var, neglectus 6-7: 28.

— clavatus $3: 23 ; 5: 81$.

- cupulatus $3: 63 ; 5: 17,81$.

- helvelloides 5: 81 .

— infundibuliformis $3: 29,50,61,67 ; 5: 81 ; 8: 48$.

- lutescens 3: 23, 50; 5: 81; 18(suppl.): 116, 117.

- tubaeformis $3: 7,29 ; 8: 46,59 ; 16: 10,12,13 ; 18: 20,21$; 18(suppl.): 41, 43, 107, 114, 115, 117.

- umbonatus $3: 7,8,16$.

Catathelasma imperiale $3: 26 ; 4: 42 ; 5: 22$.

Carcerina valvata 19: 10, 12 .

Cenangium abietis 19: 30.

— fasciculare 5: 97.

- ferruginosum 19: 30, 31.

Celluloderma 6-7: 14.

Ceraporia incarnata 8: 15 .

- viridans $f$. inconstans 8: 15.

Ceratella himantia 6-7: 29.

Ceratiomyxa 14: 79 .

— fruticulosa 6-7: 110; 14: 55; 19: 10.

- - var. porioides 19: 10 .

Ceratium aureum 19: 10 .
- hydnoides 19: 10.

- porioides 19: 10.

Cercospora ferruginea 17: 3, 14.

Cercosporella herpotrichoides 19: 55 .

- subulata 13: 9 .

Carrena unicolor 8: 15 .

Cetraria islandica 19: 20

- nivalis 19: 42.

Chaetomium 12: 59, 61.

- globosum 11: 25.

Chaetoporellus asiaticus 8: 15 .

- litschaueri 8: 15.

Chaetoporus 13: 6 .

- corticola 8: 15.

- euporus 8: 15.

- radulus 8: 15 .

Chalciporus piperatus 18: 12 .

Cheilaria agrostidis 15: 59 .

Cheilymenia 13: 52 .

Chlorosplenium aeruginascens 5: 97

Chondrioderma difforme 19: 10, 11 .

— michelii 19: 3, 10, 11 .

— radiatum 19: 10, 11 .

- testaceum 19: 10, 11.

Chrysosporium 14: 135.

- luteum 14: 135.

Cenococcum graniforme 14: 9 .

Ceratocystis ulmi 14: 32.

Ciboria 4: 28; 5: 97.

— amentacea 4: 28; 5: 97.

Ciborinia 20: 30 .

Cicinnobolus cesatii 4: 17, 22, 23; 6-7: 100.

Cintractia 17: 5 .

— andina 17: 1, 5

- carphae 17: 5.

- schoenus 17: 5 .

- waiouru 17: 5.

Cladina 2: 28; 6-7: 37, 41; 19: 22.

Cladonia 5: 114; 6-7: 82; 10: 33; 18(suppl.): 97; 19: 20.

- silvatica 5: 115 .

Cladosporium 12: 42, 53, 54, 57, 61, 62; 14: 135.

— aecidiicola 17: 14.

- herbarum 12: 53-56; 18(suppl.): 51.

- resinae 14: 135.

Clastroderma microcarpum 7: 88 .

Clavaria $1: 24,35,39,45 ; 4: 42,60 ; 5: 83 ; 6-7: 28 ; 18$ (suppl.): 114.

— abietina 3: 23, 29, 32, 42, 50,61; 5: 83.

— amethystina $3: 23 ; 5: 83 ; 6-7: 28$.

— apiculata $3: 23 ; 5: 83$.

— argillacea $3: 67 ; 5: 83$.

— aurantia $3: 67 ; 5: 83 ; 6-7: 28$.

- aurea 1: 35; 3: 23, 29, 42, 50; 5: 82 .

- botrytis $3: 23,42,50 ; 5: 82$.

- cinerea 5: 83; 6-7: 28.

- $\mathrm{f}$. sublilascens $4: 40 ; 6-7: 28$

— condensata 3: 50; 5: 82, 83; 6-7: 28.

_- var. violaceotincta $3: 23,42,50 ; 5: 83$.

- coralloides 3: 42; 5: 83 .

- corniculata $3: 42 ; 5: 83$.

- corrugata 5: 83; 6-7: 28 .

— gracilis 5: 83 .

— crispula $3: 50 ; 5: 83$.

— cristata $3: 42,50,67 ; 4: 40 ; 5: 83 ; 6-7: 28,70$.

- $\mathrm{f}$. fuligineocinerascens 6-7: 28 .

- cyanescens 3: 61; 5: 83.

- decolorans 6-7: 28 .

- decurrens 5: 83. 
— dissipabilis 3: 50; 5: 83 .

— epiphylla 9: 24.

— fastigiata $3: 67 ; 5: 83$.

— fennica 3: 50; 5: 82; 6-7: 28.

- fistulosa 3: 7, 8, 29; 5: 84; 10: 178, 180.

— flaccida 3: $29,42,50,67 ; 5: 83$.

- flava 3: 7, 23, 29, 42, 50; 5: 82 .

- formosa 1: 25, 35 .

— fragilis 3: 50,53 .

- fusiformis 3: 50; 5: 83.

— gracilis 3: 50; 5: 82, 83 .

- himantia 6-7: 29, 30.

— invalii 3: 23, 29, 42, 50, 61; 5: 83.

- juncea 5: 84 .

— kunzei 3: 29; 5: 83; 6-7: 28.

— lappa 3: 50, 67; 4: 40; 5: 83; 6-7: 28.

- ligula $3: 7,11,14,29,42,50,61,67 ; 5: 84 ; 6-7: 29 ; 9: 39$, 41.

- lilacina $3: 23 ; 5: 83$.

- luteoalba 6-7: 28 .

- mucida 5: 84; 6-7: 28.

- muscoides $3: 42,50,67 ; 5: 83$.

- - var. pratensis $3: 67 ; 5: 83$.

- nivea 5: 83 .

- pallida 17: 69 .

- phalloides 9: 24 .

- pistillaris $2: 49 ; 3: 23,42 ; 5: 84$.

— rugosa $3: 50 ; 4: 43 ; 5: 83$.

- similis 3: 50; 5: 83; 6-7: 29.

- stricta 3: 23; 5: 83 .

- suecica 5: 83.

- vermicularis 3: 50 .

— - var. fragilis 5: 83; 6-7: 28.

- - var. gracilis $\mathrm{f}$. sphaerospora 5: 83 .

— versatilis 5: 82; 6-7: 28.

— virescens 3: 61; 5: 83 .

Clavariadelphus pistillaris 17: 69 .

Claviceps purpurea 1: 39.

Clitocybe $1: 20,24,29,33,39,42 ; 2: 48: 3: 7-9,14 ; 4: 45,46$; 5: 14, 17; 6-7: 7, 8; 9: 51-53; 10: 5-14, 16-22, 24-35, 37-39. $41-43,50,55,65,68,70-72,77,82,83,89,94,101-103$, $107,108,114-117,178 ; 11: 35-37,39,40 ; 14: 50,82-90$, $113,114,121,131 ; 15: 13,14,16,17,23 ; 18: 49,50,52$, 53; 18(suppl.): 49, 51, 116, 117; 19: 22-24, 50, 51.

- aberrans 11: 36.

- absinthiata 19: 23.

- acromelalega 1: 24, 39 .

- adirondackensis 10: 81-83; 11: 40; 14: 114.

— adsentiens 10: 114 .

- aeruginosa 10: $84 ; 15: 14$.

- aggregata 2: 53 .

- agrestis 10: 19, 33-36, 40, 49, 76, 91, 92, 117, 127, 141, 148,$161 ; 15: 14$.

— alba 10: 58 .

- albida 3: 50; $5: 12$.

- alexandri 3: 20, 25, 33, 54; 5: 12; 9: 52; 10: 16, 19, 34-36, $40,47,52-55,58,114,122,131,147,150$.

- sect. Alexandriformes 10: 52 .

- altaica 10: 25, 33-36, 38, 41, 47, 61-63, 68, 134, 147, 152; 15: 20 .

- amarescens 10: 18, 19, 36, 40, 49, 98, 99, 112, 117, 128, $142,149,163 ; 15: 14$.

- ambigua 10: 114.

— angustissima $3: 26,52 ; 4: 46 ; 5: 14,15 ; 6-7: 8$.

- anisata 10: 17, 19, 25, 33, 34, 36, 40, 49,87, 89, 126, 140 , 148, 161: 18: 54

- arnoldii 19: 22 .

- aromatica 15: 16 .
- asterospora 5: 15 .

— atra 14: 126: 15: 16.

- atrostriata 14: 89.

- augeana 18: 50 .

- aurantiaca 2: 53 .

- avellaneialba 10: 59; 14: 90.

- bresadoliana 5: 13, 10: 21, 22, 28, 30, 33, 34, 36, 40, 42 , $46,61,66-71,135,147,154$

- brevipes 19: 22 .

— brumalis $3: 26,33,44,63 ; 4: 46: 5: 14,15: 10: 29: 11: 35$.

- brunnescens 11: 36 .

- sect. Bulluliferae 10: 81 .

— cacabus $3: 25 ; 5: 12 ; 11: 35$.

- caespitosa 14: 115 .

— candicans $3: 7,25,33,44 ; 4: 30,46 ; 5: 13 ; 9: 52 ; 10: 14,16$. $19,22,24-27,31,32,34,36,40,45,50,73-75,83,125$, $137,139,148,156 ; 11: 36-39 ; 19: 50$.

_ - var. dryadicola 15: 17.

— $\mathrm{f}$, phyllophila $3: 33,54 ; 4: 30 ; 5: 13,14: 10: 83$.

- carnosior 19: 50 .

— catinus (-a) $3: 52 ; 4: 30,46 ; 5: 13 ; 6-7: 7 ; 10: 18,19,23,34$. $36,46,61,63-65,134,147,153$.

— cerussata 2: 53; 3: 7, 33, 44, 52: 4: 46; 5: 13; 10: 29, 77 , $86-88 ; 11 ; 40$.

- $\mathrm{f}$. langei 4: 30 .

_- var. pithyophila $3: 25,33: 5: 13$.

- sect. Clavipedes 10: 58 .

- clavipes $2: 54 ; 3: 7,11,14,20,25,44,54 ; 4: 30,45 ; 5: 12$ : 119,$120 ; 9: 52 ; 10: 14-19,22,23,28,29,32-36,38,40,46$, $51,58-60,123,133,147,152,180 ; 11: 37,38 ; 13: 45,89$; 14: $50 ; 15: 17 ; 19: 50$.

— clitocyboides 14: 114, 15: 17.

- compressa 14: 126.

- compressipes f. autumnalis 11: 36

- concava $3: 26 ; 5: 14 ; 6-7: 8$.

- conglobata $2: 54$.

- connata 2: 54: 3: 25 .

— corda 10: 76 .

- costata 4: 30:5: 13:11: 35 .

- crispa 10:61: 15: 17, 18.

- cyathiformis 2: $54: 3: 26$.

- dealbata 1: 20, 25, 29, 36, 39, 42; $2: 54 ; 3: 25,33 ; 4: 30,46$; 5: 13:10: 16, 33-36, 40, 50, 75-77, 87, 92, 137, 148, 157.

— diatreta $2: 54 ; 3: 26,44,63 ; 4: 46 ; 5: 14,15 ; 10: 17,19$. $33-36,40,49,77,83,86,90-93,114,127,140,148,161$ : 18: 49; 19: 22,50 .

— dicolor 3: 26. 33, 44: 4: 30, 46:5: 14, 15; 6-7: 8; 10:97, 142

- ditopa 2: 54:3:33, 63:4:42, 46; 5: 14:10:21, 22, 101, 102 . 106, 107; 11: 36 .

- - var. ditopa 10: 14, 15, 19, 28, 32, 34, 36, 39, 40, 48, $102,105,106,129,144,149,166 ; 11: 36,37$.

- - var. odorula 10: 19, 31, 33-36, 40, 48, 106, 107, 118, $129.144,149,166$.

- dryadicola 15: 16, 17.

- subsect. Epruinatae 10: 90.

- ericetorum $3: 63 ; 5: 13$.

- expallens $3: 26,30 ; 5: 14$.

- - var. trivialis 10: 101 .

- fallax 11: 36 .

- fasciculata 11: 39.

- favrei 6-7: 8.

- fennica 10: 22, 23, 36, 40,48, 111, 112, 117, 130, 146, 149 , 168.

- festiva 18: 50.

- festivoides 18: 50 .

— flaccida 3: 44; 4: 46; 5: 13; 10:67, 68 .

- fragrans $2: 54 ; 3: 20,25,33,54: 4: 30,46 ; 5: 14: 10: 18-20$. $22,25,32,34-36,39,40,49,83,93-95,127,141,148,162$; 
11: $36 ; 15: 13,14 ; 18: 54$.

- sect. Fragrantes nom.prov. 10: 93.

— fritilliformis $3: 26 ; 4: 46 ; 5: 14: 6-7: 8$.

— fuligineipes $4: 46 ; 5: 14 ; 6-7: 8 ; 10: 18,112$.

- fumosa 3: 7, 11, 14 .

- gallinacea 10: 82; 14: 114.

- geotropa 2: 54; 3: 20, 63; 5: 12; 6-7: 7; 10: 17, 18, 21, 23, $25,28,32-36,38,40,42,46,60-63,117,124,133,147$, 152; 15: 19; 18: 29 .

_ - var. involuta $3: 30 ; 4: 62$.

_- var. subinvoluta 3: 44; 4: 62 .

— gibba 9: 53; 10: 14, 18, 19, 30, 31, 34-36, 40, 47, 60, 61, $63-67,124,134,147,153 ; 11: 37 ; 14: 85-87 ; 15: 28 ; 17: 67$; 18: 49 .

- gigantea $2: 54 ; 10: 114$

- gigas 18: 29 .

- gilva $2: 54 ; 3: 7,20,44,52 ; 5: 12 ; 6-7: 7 ; 10: 68,71 ; 14$ : $82-84,114 ; 15: 14 ; 18: 49$.

- - var. lapponica 10: 68 .

- sect. Gilvaoideae 10: 70.

- gilvaoides 10: 36, 40, 46, 70-72, 136, 147, 156.

- - var. gilvaoides 10: 71 .

— - var. gracilis 10: 72 .

- glaucoalba 10: 84 .

- globispora 10: 20, 22, 27, 34, 36, 40, 48, 100, 101, 117, 143 . $149,164$.

- gossypina 11: 36 .

- gracilis 10: 17, 22, 23, 32, 36, 40, 46, 71, 72, 118, 136, 147, 156.

- griseifolia 19: 22 .

- harmajae 15: 14

- harperi 10: 25, 27, 28, 32, 34, 36, 40, 47, 51, 52. 54, 55, 114. 131, 147, 150; 14: 90; 15: 21; 18: 30; 19: 22.

— highlandensis 14: 90; 19: 24.

- hirneola 3: 45 .

- hydrogramma $3: 44,54 ; 4: 42,46 ; 5: 14 ; 10: 23,26,36,40$, $48,51,75,81-83,95,108,139,148,159 ; 11: 40 ; 14$ : $113-115,15: 16,17 ; 18: 49$.

— - var. gibboides 10: 83; 14: 115 .

- - var. werneri 14: 115.

- idahoensis 15: 14.

- incisa 11: 36 .

— incomis 19: 22, 23.

- infundibuliformis $2: 54 ; 3: 7,14,20,25,30,33,44,52,54$; 4: 30,$45 ; 5: 13,119,120 ; 6-7: 7 ; 10: 64,65,67,134 ; 17$ : 67; 18(suppl.): $26,27$.

- - var. catinus 5: 13; 6-7: 7 .

- - var. splendens 5: 13 .

- incarnata 6-7: 8 .

_ inornata 3: 33, 52; 5: 12; 9: 52; 10: 15, 16, 19, 20, 22, 32 . $34-36,38,40,47,53,55,114,122,132,147,151 ; 11: 40$.

— inversa $2: 54 ; 3: 7,11,14,25,44,63 ; 4: 46 ; 5: 13 ; 10: 9$.

- $-\mathrm{f}$. pallidispora $3: 63 ; 10: 114$.

- kuehneri 10: 81, 82: 14: 113, 115 .

- laccata 2: 54.

- langei $3: 63 ; 4: 42,46 ; 5: 14: 10: 17,19,22,23,31,33-36$, 40. 48, 102-105, 129, 144, 149, 165 .

- lapponica 10: 22, 34-36, 38, 41, 47, 61, 63, 66-68, 117, $135,147,155 ; 15: 19,20$.

- Laricicola 10: 16, 20, 33-36, 38, 41, 50, 73, 75-77. 84, 137 . 148. 157.

- sect. Latisporae 10: 100.

- lauta 10:114.

- lentiginosa $3: 44: 5: 13$

- leucopaxilloides 19: 24

- Ieucophylla $3: 63: 5: 14$.

- lignatilis 10: 9.

- lituus 6-7: 8 .
- lohjaënsis 10: 17-20, 22, 23, 26, 32, 34-36, 40, 48, 108, 109. $112,113,117,130,146,149,168 ; 15: 27,28 ; 18: 29$.

- macrophylla 10: 114 .

- marginata 10: 93.

- marginella 10: 17, 19, 32-36, 40, 49, 77, 92, 117, 127, 141, 148,$162 ; 15: 14$.

- maxima 6-7: 7 .

- media 15: 17.

- menthiodora 10: 15, 18, 19, 23, 34, 36, 40, 48, 102, 107. $117,129,145,149,167$.

- metachroa $2: 54 ; 3: 7,26,63 ; 4: 46 ; 5: 15 ; 6-7: 8 ; 10: 19,22$, $32,34-36,40,49,95-99,103-105,112,128,142,149,163$.

- metachroides 10: 33, 34, 36, 40, 49, 96, 98, 99, 117, 142. 149, 163; 15: 14 .

- microspora 18: 50.

- montana 15: 19, 20

- morbifera 10: 76.

- mortaricola 11: 36,37 .

- mortuosa 11: 35 .

- murinifolia 11: 37.

- nauseosodulcis 10: 115 .

— nebularis $1: 16,18,85 ; 2: 54 ; 3: 25,33,63 ; 4: 60 ; 5: 12,89$, $107 ; 8: 47-49,60 ; 10: 14,16,18,19,23,28,29,32,34-36$, $38,40,46,52,56,57,60,65,123,132,147,151,180: 13$ : $45,84-87 ; 14: 114,129,130,131 ; 15: 14: 16: 12 ; 18: 49$; 18(suppl.): $23,24,26,27,34-37,41$.

- - var. alba 6-7: 7; 10: 58 .

- - ssp. (var.) stenophylla 10: 57.

- novembrina 11: 37 .

- nybergii 10: 115 .

- obolus 3: 26; $5: 15$.

- obscurata 11: 37.

—obsoleta $3: 20,25,33 ; 4: 30 ; 5: 14 ; 10: 94$.

- odora 2: 54; 3: 7, 11, 14, 20, 25, 33, 44, 54; 4: 29, 45; 5: 119,$120 ; 6-7: 7,70 ; 10: 14,18,19,25,26,29,30-36,40$, $46,65,83,84,86,87,125,139,148,159,180 ; 13: 45,87$; 14: $131 ; 18: 49 ; 19: 50$.

— - var. alba $3: 33,44 ; 4: 30,45 ; 5: 12$

— anisaria 19: 50 .

- sect. Odorae nom. prov. 10: 84.

— odorula 10: 106.

— olida 11: 37

—olorina $4: 30,46 ; 5: 14 ; 6-7: 7 ; 11: 35$.

- orbiformis 5: 15; 11: 35 .

- orientalis 10: 22, 23, 34-36, 40, 48, 102-104, 117, 143, 149, 164.

- pallens 10: 115 .

- pantoleucoides 10: 115 .

— parilis 6-7: 7; 10: 83 .

- paropsis 6-7: 7; 11: 35 .

- pausiaca 11: 35 .

- phyllophila $2: 54 ; 3: 20,25,33,44,54 ; 4: 30,46 ; 5: 13 ; 10$ : $16,76,77,83,84,86-89,180 ; 11: 40 ; 14: 86$.

- - var. phyllophila 10: 17, 19, 28, 29, 32-36, 40, 49, 85-89, $114,116,126,139,148,160,11: 37,40$.

- - f. pithyophila 10: 87 .

- - var. tenuis 10: 19, 31, 33-36, 40, 49, 87-89, 117, 126, $140,148,160 ; 15: 15$.

— phyllophiloides 19: 50 .

— pinetorum 4: 30, 46; $5: 14,15 ; 6-7: 8 ; 10: 91$.

— piperata 14: 121; 19:22, 24 .

— pithyophila $3: 25,33,44,54 ; 4: 42 ; 5: 13 ; 6-7: 7 ; 10: 87$

- polygonarum 18: 50 .

- pometi var. saliceti 10: 115

- praecox 11: 37.

- subsect. Pruinatae 10: 86

- pruinosa 10: 14, 16, 19, 22, 27, 28, 31, 33-36, 38-40, 45, 47, $73,77-81,115,125,138,148,158 ; 11: 38$. 
- pseudoirina 14: 83, 90; 15: 17

- pseudoobbata 6-7: 8 .

- puellula 10: 114, 115 .

— pusilla 19: 50,51 .

— radicellata 5: 12,$13 ; 6-7 ; 7 ; 9: 52 ; 10: 79 ; 11: 38$.

- raphaniolens 10: 96.

- regularis $1: 20,25,29,36,39,42 ; 2: 54 ; 3: 20,25,44,54$; 10: $89 ; 15: 15$.

- rhizoides 11: 38.

- rhizophora $3: 25,54 ; 4: 27,28 ; 5: 12 ; 10: 15,16,19,20,21$, $28,31,34,36,38-40,47,78-81,115,125,138,148,158$.

- rigidata 10: 115 .

- rivulosa $1: 20,25,29,36,39,42 ; 2 ; 54 ; 3: 20,25,44,54 ; 4$ : $30,46,5: 13 ; 10: 73,76$.

- - var. dryadicola 15: 17 .

— robusta $10: 58 ; 15: 15$.

- subg. Roseospora 10: 83.

— rubella 18: 50 .

- ruderalis 10: 33-36, 40, 50, 75, 76, 117, 137, 148, 157.

- rufoalutacea $4: 45 ; 5: 13$.

- saliceti 10: 115 .

- schulmannii 10: 18, 22, 36, 40, 47, 109, 112, 117, 146, 149, 168; 14: 84; 19: 50, 51 .

- sericella $4: 45 ; 5: 13 ; 6-7: 7 ; 11: 35$.

- serotina 18: 29.

- sinopica 2: 54; $3: 20,33,44 ; 4: 30 ; 5: 13 ; 6-7: 7 ; 10: 14,16$, $19,21,28,33-36,38-40,46,61,69,70,81,125,136,147$, 155; 11: 36-38; 18: 29; 19: 22.

— - var. arnoldii 19: 22.

— - var. sinopicoides $3: 44 ; 5: 13 ; 6-7: 7$.

- sect. Sinopicae 10: 69.

- sinopicoides 4: 46.

- socialis 10: 61 .

- splendens 3: 52; 5: 13.

- squamulosa $2: 54 ; 3: 20,25,54 ; 4: 45 ; 5: 13 ; 6-7: 7 ; 10: 14$, $18,19,21,23,30,32-36,39,40,46,61,65-68,110,124$, $134,147,154 ; 11: 35$.

- squamulosoides 11: 37,38

- stenophylla 10: 57.

- sect. Strigipedes 10: 108.

- strigosa 10: 15, 17, 19, 22, 23, 25, 26, 30, 32-36, 40, 47, $109-111,117,130,145,149,167 ; 14: 114 ; 18: 29,54 ; 19$ : 42.

- suaveolens $3: 20,25,33 ; 4: 30,46 ; 5: 14 ; 10: 29,73,94 ; 18$ : 54.

- subalpina 14: 91.

- subalutacea 4: 45; 5: 13; 6-7: 7 .

- subconnexa 11: $38 ; 14: 86$.

- subcordispora 10: 17, 22, 23, 31, 33-36, 40, 48, 100, 117, $128,143,149,164$

- subdryadicola 18: 29.

- subfumosa 19: 50, 51 .

- subinversa 10: 72 .

- subinvoluta 5: 12; 11: 37 .

- subnitens 15: 17, 18.

- subsericella 11: 35 .

- subsinopica 18: 29.

- subviscifer 10: 113.

— sudorifica 1: 29.

- tenuissima 5: 13; 10: 75; 11: 35, 39.

- tornata 11: 35 .

- trogii 4: 30 .

— tuba 3: 33, 44, 54; 5: 13; 6-7: 7; 11: 35.

— umbilicata 5: 14; 6-7: 8 .

- umbonata 2: 54 .

— umbrinipes 11: 40.

— umbrinomarginata 4: 31; 5: 25.

— vasilievae 15: 18.
— vermicularis $3: 54 ; 5: 13 ; 4: 27,28 ; 10: 80,81 ; 11: 35$.

— verna 10: 79

- sect. Vernae 10: 79

- vernicosa 5: 13 .

— vibecina $3: 26,33 ; 4: 46 ; 5: 14 ; 10: 17,23,24,28,33-36,40$, $48,97,102-105,108,129,144,149,165$.

- - var. floccipes 6-7: 8 .

— viridis 10: 29.

- washingtonensis 11: 40 .

Clitopilopsis 5: 20 .

— fallax 3: 55; 5: 29 .

— hirneola 3: 45; 5: 29; 6-7: 10, 12; 10: 101 .

— mundula $3: 27 ; 5: 29$.

- popinalis $5: 29 ; 6-7: 12$.

Clitopilus 1: 31; 2: 61; 5: 15;6-7: 11; 14: 89.

— cretatus 5: 30,39 .

- giovanellae 4: 32; 5: 29.

— omphaliformis 5: 29 .

- prunulus $2: 54 ; 3: 7,8,12,14,21,27,31,34,45,55 ; 4: 32$, $47,48,60 ; 5: 20,29 ; 6-7: 9,12,60,64,70 ; 10: 180$; 18(suppl.): 41, 116, 117.

- scyphoides 3: 27, 33, 34.

- submicropus 5: 29.

- undatus 17: 63 .

Coccidiodes immitis 15: 10 .

Cocliobolus sativus 12: 26.

Coleosporium campanulae 6-7: 95.

Colloderma 17: 31 .

Collybia 1: 20, 26, 29, 42, 78; 3: 14, 44, 63; 4: 45; 5: 16, 21 , 114,$119 ; 6-7: 9,12 ; 10: 7,178 ; 14: 87-89 ; 17: 63 ; 19: 26$.

— acervata $2: 54 ; 3: 26,44,54 ; 4: 46 ; 5: 16 ; 18$ (suppl.): 41 .

- ambusta 5: 11, 105 .

— aquosa $3: 30,33,44,54 ; 4: 30,46 ; 5: 16 ; 6-7: 9$.

— asema $3: 20,26,34,54 ; 4: 30,46 ; 5: 16 ; 10: 108,182 ; 13$ : $45 ; 14: 50,51$.

- butyracea 1: 29, 42; 2: 54; 3: 7, 12, 14, 26, 34, 44, 54,63; 4: $10-13,30,46 ; 5: 16,17,102,112,119,120 ; 9: 40,41 ; 10$ : $180 ; 12: 62,63 ; 14: 50,51,88,89 ; 18$ (suppl.): $41 ; 19: 59$. 60 .

— cirrhata $2: 54 ; 3: 44,54,63 ; 4: 36,46 ; 5: 15 ; 6-7: 8 ; 14: 50$. 51.

- clusilis 5 : 11 .

- confluens $2: 54 ; 3: 7,12,14,26,33,44,54 ; 4: 44,46 ; 5: 16$, 119,$120 ; 6-7: 4,49 ; 10: 180 ; 12: 63 ; 13: 45$.

— conigena $2: 28-30,54 ; 5: 29 ; 6-7: 8,9$.

- cookei 2: 54; 6-7: 8, 9 .

— distorta 2: 54, 3: 30, 44; 4: 46; 5: 17; 14: 88

- dryophila $2: 54 ; 3: 7,12,14,20,26,30,33,44,54 ; 4: 30$. $46 ; 5: 16,119,120 ; 6-7: 7,9,37 ; 9: 40-42 ; 10: 180,182 ; 11$ : $25,26,36 ; 13: 45 ; 14: 49-52$.

— - var. exsculpta 5: 16; 6-7: 9 .

_ - var. funicularis $3: 33 ; 4: 30,46 ; 5: 16$.

- - var. oedipus 4: $46 ; 5: 16$.

_ - var. xanthochroa 4: 46; 5: 16 .

- erosa $3: 63 ; 4: 29$.

- erythropus $4: 44 ; 5: 16$.

— esculenta $2: 28,29 ; 3: 45$.

- exsculpta 4: 46 .

- extuberans 4: 46; 5: 16.

- funicularis 3: 54 .

- fuscopurpurea $3: 20 ; 5: 15 ; 6-7: 9 ; 17 ; 59,67$.

- fusipes 6-7: 47 .

- hariolorum $3: 45 ; 5: 16 ; 6-7: 9$.

- impudica 5: 15; 6-7: 9 .

- incomis 19: 22-24.

— ingrata $5: 16 ; 6-7: 9$.

- leucomyosotis 5: 11 .

— macilenta $3: 44,54 ; 5: 16$. 
- maculata $1: 29 ; 2: 54 ; 3: 7,26,30,34,44 ; 5: 16 ; 14: 50,51$, $88 ; 18$ (suppl.): 41.

- - var. scorzonera 5: 16

- maculosa 4: 30; 6-7: 70 .

- myosura 2: 30 .

- nummularia 3: 44; 5: 16.

- obscura 17: 67.

—ocellata $3: 44,54 ; 4: 46 ; 5: 16 ; 6-7: 7,9$.

— peronata $2: 54 ; 3: 33,44,63 ; 5: 15 ; 6-7: 8 ; 10: 180 ; 17: 67$.

- platyphylla $2: 54 ; 3: 7 ; 6-7: 69$.

- prolixa 3: 63; 4: 46; 5: 17 .

- pudens 6-7: 46 .

— putilla $2: 54 ; 3: 26,30 ; 4: 46 ; 5: 15 ; 6-7: 9,11 ; 10: 180 ; 14:$ 49-51.

— radicata 6-7: 47, 69 .

- rancida 3: 7, 12, 14 .

- rhizophora 10: 80,81 .

- strumosa 4: 46; 5: 16; 6-7: 12.

- tenacella $2: 29,54 ; 3: 7,14,45$.

- tesquorum $3: 44,63 ; 4: 29$.

— tuberosa $2: 54 ; 3: 63 ; 4: 30,42,46 ; 5: 15$.

- tylicolor 3: 63; 4: 29.

- velutipes 16: 3 .

- urens 2: 54 .

— xanthopus $3: 44 ; 5: 16 ; 6-7: 9$.

Coltricia 17: 34 .

- cinnamomea 8: 15 .

- perennis 8: 15 .

- spathulata 17: 33,34

Comatricha 17: 31,89

— alta 19: 1, 2, 10 .

- dictyospora 19: 6 .

— elegans 17: 19, 24, 88, 89; 19: 10 .

- - var. microcarpa 17: 88 .

- ellae 18: 23; 19: 10, 14.

- fimbriata 17: 26.

- friesiana 19: 10.

- irregularis 14: 67, 78; 19: 1, 6, 7, 10, 13, 16 .

— laxa 14: 67, 68; 17: 88, 89; 19: 10 .

— nannengae (-iae, -ii) 17: 87-89; 18: 23; 19: 10, 14

- nigra 6-7: 110; 14: 66, 67, 80; 17: 19, 24, 25, 29-31, 87-89; 18: $60 ; 19: 10,13$.

— - var. alta 19: 2 .

- - var. laxa 14: 67.

— rigidireta 17: 88

- solitaria 17: 26.

- typhina 19: 10, 13.

- typhoides 14: 67; 19: 10, 13.

Coniophora 1: 61, 62, 68-71.

— arida 5: 86

- betulae 5: 86 .

— cerebella 1: 61, 68-71; 5: 107.

- macra 5: 86.

- subcinnamomea 5: 86.

Coniothyrium bergii 17: 14 .

Conocybe 2: $56 ; 4: 35$.

— apala var. sphaerobasis 4: 54; 5: 63.

- coprophila 17: 59,61.

— grisea 3: 22; 5: 63.

- lactea $3: 39 ; 4: 37,54 ; 5: 63 ; 17: 61$.

— - f. semiglobata 5: 63 .

- lateritia 3: 39; 4: 37; 5: 63 .

- magnicapitata 17: 61 .

- mesospora 5: 63; 17: 61.

- neoantipus 5: 63 .

- plumbeitincta 4: 54; 5: 63 .

- pubescens 17: 59,61.

- rickeniana $4: 37,54 ; 5 ; 63$.
- $\mathrm{f}$. sordida 6-7: 24

— rickenii 3: 40; 5: 63 .

- siliginea 3: 22, 40; 5: 63 .

—-var. ochracea 4: 54; 5: 63 .

- spicula 4: 37; 5: 63 .

- tenera $2: 54 ; 3: 22,28,31,39,48,59 ; 4: 37,54 ; 5: 63$.

- -f. convexa 3: 48; 5: 63; 6-7: 24 .

- - var. macrocephala 5: 63 .

— - var. (f.) minor 3: 48; 5: 63; 6-7: 24 .

_- var. semiglobata $3: 59,63 ; 4: 44,54 ; 5: 63 ; 6-7: 24$.

— - var. subovalis 3: 59; 4: 37, 54; 5: 63; 6-7: 24.

- - var. (f.) tenella $3: 22,40 ; 5: 63$.

- teneroides 5: 63.

Copelandia 6-7: 26 .

- cyanescens 5: 69; 6-7: 26.

- papilionacea 5: 69, 70 .

- solidipes 6-7:26.

- teutonica 6-7: 26

Coprinus 1: 32,$43 ; 2: 54 ; 5: 74,101-103,105,112,117 ; 6-7$ : 82; 10: 24; 18(suppl.): 54 .

— angulatus $3: 28,49 ; 5: 74$

- atramentarius $1: 15,24,32,36,43 ; 2: 49,54 ; 3: 22,31,40$, 49, 73; 4: 39; 5: 74; 18(suppl.): 41, 116, 117.

- - var. acuminatus 4: 57; 5: 73.

— auricomus $3: 67 ; 5: 75$.

- boudieri 4: 39, 57; 5: 75, 100-105.

- cineratus $3: 54 ; 5: 74$.

- cinereus 5: 73

- comatus 2: 54; $3: 22,28 ; 4: 60 ; 5: 73 ; 9: 40-42 ; 12: 55$; 18(supp.): 43, 45, 114, 115, 117.

— cordisporus $3: 28,49 ; 4: 57 ; 5: 74$.

- cortinatus 4: 61 .

- crenatus 5: 75 .

- curtus 4: 39; 5: 75; 6-7: 27.

- disseminatus 5: 75.

-domesticus 3: 22, 41, 49, 53, 60; 4: 39, 57; 5: 74.

- ephemeroides 5: 74 .

- extinctorius 5: 74 .

- fimetarius $2: 54 ; 3: 28,40 ; 5: 73$.

- - var. macrocephalus 3: 40; 5: 73.

- flocculosus 5: 74.

- friesii 3: 49, 53; 4: 39, 57; 5: 74

- fuscescens $3: 22,28,31,40 ; 4: 39 ; 5: 73$.

- hansenii 5: 75 .

- hemerobius $3: 67 ; 5: 75$.

- impatiens 4: 57; 5: 75 .

- lagopus $3: 41,67 ; 5: 73 ; 13: 37 ; 16: 3$.

- - var. sphaerosporus 5: 74 .

- macrocephalus $3: 40 ; 5: 73 ; 6-7: 27$.

- macrorhizus 5: 73 .

— micaceus $1: 24,32 ; 2: 54 ; 3: 22,28,41,60 ; 4: 39 ; 5: 74 ; 11$ : 24-26; 18(suppl.): $34,93$.

- miser 4: 61; 5: 75.

— narcoticus 4: 39; 5: 74 .

— niveus $3: 22,41,60 ; 4: 39,57 ; 5: 74 ; 17: 62$.

- nycthemerus 4: 39; 5: 74.

- ovatus 5: 73.

— papillatus $3: 28,49 ; 5: 74$.

— patouillardii 3: 28, 49; $4: 57 ; 5: 74$.

— pellucidus 6-7: 27 .

- phaeosporus $3: 53 ; 4: 39,57 ; 5: 74$.

- - f. solitarius $5: 74$.

- picaceus 6-7: 27.

- plicatilis $3: 41,60,67 ; 4: 39 ; 5: 75 ; 6-7: 27$.

- - var. microsporus 5: 75 .

— radians 6-7: 27 .

— radiatus 3: 53; 4: 39; 5: 73.

— radicans $4: 39 ; 5: 74$. 
- roris $4: 61 ; 5: 74 ; 6-7: 27$.

- rostrupianus $4: 57 ; 5: 74 ; 6-7: 27$.

- similis 6-7: 27.

- stercorarius 3: 28, 54; 4: 39; 5: 73, 74.

- tomentosus 4: 39; 5: 74 .

- truncorum 3: 60; 4: 57; 5: 74 .

- velatus 5: 74 .

- velox 3: 28; 4: 57; 5: 74 .

- xanthothrix 3: 22, 41; 4: 57; 5: 74.

Cordyceps 4: 41; 5: 98 .

— militaris 4: 41, 43; 5: 98.

- ophioglossoides 6-7: 34 .

- typhina 15: 50.

Coriolellus 18: $43,46,48$.

- heteromorphus 8: 15; 10: 182.

— malicola 18: 47 .

- salicinus 18: 48 .

— serialis 8: 15; 10: 182; 11: 29, 31; 12: 47-50.

Coriolus 12: 64 .

— abietinus $3: 24,32,42,51,54,61 ; 4: 41,59 ; 5: 90 ; 8: 17$.

- $-\mathrm{f}$. resupinatus 5: 90 .

- cinere us 6-7: 31 .

- connatus $3: 24 ; 4: 43 ; 5: 90$.

— fibula 4: 59 .

— hirsutus $3: 24,42,61 ; 4: 41,43,59 ; 8: 15 ; 10: 181,182 ; 11$ : $31 ; 12: 47-50,62,64 ; 13: 45,46$.

— - var. fibula 4: 41, 5: 90; 6-7: 31 .

- hoehnelii 6-7: 31; 8: 15 .

- neesii 4: 41 .

- - var. alneus 4: 41, 59; 5: 90.

- populinus $4: 43 ; 5: 90 ; 6-7: 31$.

- pubescens $3: 24,42,51 ; 4: 41,59 ; 5: 90 ; 6-7: 31 ; 8: 15$.

— unicolor $3: 42,51,61 ; 4: 41,43,59 ; 5: 90 ; 6-7: 31$.

— vaporarius $8: 16 ; 10: 182 ; 11: 29,31 ; 12: 47-51$.

— velutinus $3: 24,32,42 ; 4: 41,59 ; 5: 90 ; 8: 16$

— versicolor $3: 24,29,32,51,61 ; 4: 41,59 ; 5: 90$.

- - f. fuscatus 6-7: 31 .

- zonatus $3: 24,29,32,42,51 ; 4: 41,59 ; 5: 90 ; 8: 16 ; 12$ : 47-49.

Cornuvia 19: 14.

- anomala 19: 10, 14.

Corticia 5: 5 .

Corticium 4: 58; 5: 84-86; 6-7: 29, 30; 14: 9.

- bicolor 14: 9 .

— bombycinum 5: 84; 6-7: 29.

- byssinum 6-7: 29.

- calceum 5: 84.

- confluens 4: 61; 5:84, 86.

- - var. subcalceum 5: 85 .

- crustaceum 5: 84 .

- deflectens 5: 84

- hypnophilum 5: 84.

- karstenii 5: 84.

- lacteum 4: 61; 5: 84; 6-7: 29.

- laetum 5: 84.

- laeve 4: 43, 58; 5: 84.

- microsporum 6-7: 29.

- molle 5: 84; 6-7: 30.

- myxosporum 5: 85 .

- padinum 5: 84 .

- pelliculare var. merulioides 6-7: 29.

- roseum 4: 61; 5: 84 .

- serum 5: 84 .

- tuberculatum 5: 84.

- vellereum 6-7: 29

- xerocarpum 5: 84 .

Cortinarius $1: 18,25,30,36,42,46 ; 2: 52,55,56,60,61,64$; $3: 6,8,9,14,16 ; 4: 42,50 ; 5: 43 ; 6-7: 68 ; 9: 51 ; 10: 169$;
14: 11, 49-52; 16: 10; 18(suppl.): 41, 49, 51 .

- alboviolaceus $2: 55 ; 3: 22,27,31,37,47,57 ; 4: 36 ; 14: 50$.

— anomalus 2: 55, 57; 3: 22, 37, 47, 57; 14: 50.

- - var. lebretonii 4: 36 .

- var. pineti 3: 27.

- arenatus 4: $36 ; 5: 51$.

— armillatus $3: 7,14 ; 6-7: 37 ; 8: 39,47,48,59 ; 14: 11,50$; 18(suppl.): 29-31, 41, 116, 117.

- bolaris 2: $55 ; 3: 7$.

- bovinus 18(suppl.): 41 .

- brunneus 3: 7, 8; 14: 50, 51.

- bulliardii 3: 47 .

— callisteus 3: 22, 31, 37, 47, 57, 58; 5: 51; 6-7: 19.

- $\mathrm{f}$. limonius 3: $37 ; 5: 51$.

- camphoratus 2: 55; $3: 7,8,22,27,31,37,47,57 ; 4: 36 ; 14$ : 50.

- caninus 3: 27, 37.

— cinnamomeus 3: 7; 6-7: 37; 14: 11; 17: 63 .

- collinitus $3: 7,8 ; 14: 49,51$

- cotoneus 17: 63 .

- distans var. olympicus 6-7: 19.

- elatior 3: 7 .

— gentilis $2: 55 ; 3: 7,27,37,47,57,66 ; 4: 36,51 ; 5: 51,6-7$ : 19; 14: 50 .

- hemitrichus 9: 40-42, 45; 14: 50.

- hircinus 3: 52, 57; 4: 36 .

- kauffmanianus 4: 36 .

- lepidopus 4: 36.

- limonius 2: 55; 5: 51: 6-7: 19.

- malachius 2: 55; 17: 63 .

- malachoides 17: 63 .

- malicorius 17: 63.

- melanotus 4: 36; 5 : 51 .

— olivaceofuscus 17: 59,63.

— opimus 3: 37, 47.

- orellanus 18(suppl.): 7 .

— pholideus 2: 55; $3: 7,27,37,57 ; 4: 36 ; 14: 50$.

- pseudobolaris 3: 66 .

- raphanoides $3: 22,27,31,37,47,57 ; 4: 36,51 ; 5: 51 ; 6-7$ : 19.

- rubicundulus 3: 66 .

- sanguineus $3: 7$.

- saniosus 5: 51 .

- semisanguineus $3: 7,12,14 ; 14: 9-11$.

- speciosissimus 6-7: 19; 18(suppl.): 40-42.

- speciosus 6-7: 19.

- spilomeus 2: 55; 3: 32, 37, 57; 4: 36.

- - var. depauperatus $3: 37,57$.

- suillus 3: 22.

- tophaceus 4: $36 ; 5: 51 ; 6-7: 19$

- traganus $2: 55 ; 3: 7,8,22,27,32,37,47,57 ; 14: 50$.

- triumphans 18(suppl.): 29, 31, 116, 117.

- venetus 3: 22; 5: 51 .

- violaceocinereus 3: 31, 37, 47.

— violaceus $2: 55 ; 3: 7,22,32,37,57 ; 5: 51 ; 18$ (suppl.): 41 .

Coryne sarcoides 4: 41, 60; 5: 97.

Corynetes arenarius 6-7: 112; 9: 8 .

Craterellus cinereus 6-7: 67, 69 .

- cornucopioides $2: 48 ; 3: 29,41,50,61 ; 5: 81 ; 6-7: 44,60$ $66,67,70,73 ; 16: 10,12,13 ; 17: 69 ; 18$ (suppl.): $6,43,115$, 117.

- crispus 6-7: 28, 66, 73

- pallidus 3: 50; 5: 81; 6-7: 28.

- sinuosus 6-7: 28, 44, 60, 66, 67, 69; 8: 12.

Craterium leucocephalum 14: 61, 79; 19: 10.

- minutum 14: 61; 19: 10.

- pedunculatum 19: 10 .

Crepidotus 1: 31 . 
- amygdalosporus 6-7: 15.

_ applanatus 2: 55; 3: 31; 4: 42; $5: 39$.

— bresadolae 4: 49; 5: 39.

— calolepis $3: 21,52 ; 5: 39 ; 6-7: 15$.

- cesatii 5: 39.

- fulvotomentosus $3: 21 ; 5: 39 ; 6-7: 15$.

- haustellaris 6-7: 15 .

- lundellii 5: 39; 6-7: 15 .

— mollis 2. 55; 3: 27; 4: 33; 5: 39; 6-7: 15; $17: 63$.

— pubescens 4: 49 .

— scalaris $3: 31 ; 4: 42 ; 5: 39$.

- sphaerosporus 5: 39 .

— variabilis $2: 55 ; 3: 27 ; 4: 33 ; 5: 39 ; 6-7: 15$.

- - var. subsphaerosporus 3: 46, 65; 6-7: 15.

Cribraria 14: 70, 79 .

— argillacea 14: 69; 19: 10 .

— aurantiaca 14: 69, 70; 19: 2, 10, 14.

— cancellata 19: 10 .

- intricata 14: 70; 19: 10 .

- microcarpa 14: 70; 19: 10.

- piriformis 14: 70; 19: 10 .

— purpurea 14: 70; 19: 2, 10.

— rufa 19: 2, 10.

— splendens 14: 70; 19: 10.

— tenella 14: 70; 19: 10 .

- vulgaris 14: 69, 70; 19: $2,10,14$

- - var. aurantiaca 14: 69, 70.

Crinipellis 4: 6 .

Crucibulum vulgare 3: 29, 41, 50; 5: 93.

Crumenula 2: 36, 38 .

- abietina 2: 36, 38 .

- sororia 2: 38 .

Cudonia 14: 49 .

— circinans 3: 30, 68; $4: 59 ; 5: 95 ; 14: 51$.

- confusa $3: 24,30,42,52,62 ; 4: 41,59 ; 5: 95,119,120 ; 14$ : $49,51$.

Cyathipodia platypodia 19: 44 .

Cvathus striatus 5: 93; 6-7: 32, 67; 17: 70 .

Cylindrocarpon 12: 59 .

Cyphella $4: 40 ; 5: 5$.

Cystoderma 2: 58; 3: 14; 4: 49; 5: 38, 39; 6-7: 15; 19: 25-29, 52.

— adnatifolium 14: 121, 122; 19: 29.

- ambrosii 19: 28, 29.

- amianthinum 2: 56; $3: 7,8,14,21,27,35,46,56 ; 4: 33,49$; 5: 38,$39 ; 6-7: 37 ; 9: 40,41 ; 10: 180 ; 14: 49-51 ; 19: 25-29$.

— - var. longisporum 5: 38; 18: 29; 19: 25, 26.

- - f. rugosoreticulatum 5: 38 .

- var. sublongisporum 19: 25.

- carcharias 2: 56; 3: 7,8, 12, 14, 27, 35, 46, 56, 65; 4: 33, 46, $47,49,53,55 ; 5: 13,14,19,36,38,39,56,57,60,62,66$, 119,$12 ; 6-7: 8,15,21,23 ; 10: 17,48,49,95,96,98,99$, 109,$110 ; 18: 53 ; 19: 28,29$.

- cinnabarinum $2: 56 ; 3: 21,27,35,46,56 ; 4: 33,49 ; 5: 38$, 39; 14: $122 ; 18: 30 ; 19: 25,27,28$

_fallax 4: 49; $5: 38,39 ; 19: 25,27,29$.

- granulosum 2: 56; 3: 7, 12, 14, 21, 27, 35, 46; 4: 49; $5: 38$ $39 ; 6-7: 70 ; 10: 180 ; 14: 50,122 ; 19: 27-29$.

- intermedium 19: 25, 27-29.

- jasonis 18: 29; 19: 25-27, 29.

- lilacipes 18: 29; 19: 25-27, 29.

- longisporum 19: 25.

- - var. purpurascens 18: 29; 19: 25-27

- sublongisporum 19: 25.

- terreii 18: 29, 30; 19: 25, 27, 29.

- tuomikoskii 19: 25, 27, 29.

Dacryomyces 3: 61 .

- abietinus 5: 82 .
- cerebriformis 5: 82 .

- conformis 5: 82 .

- deliquescens 6-7: 32 .

- lutescens 4: 40; 5: 82; 6-7: 32 .

- radicatus 5: 82 .

- stillatus 3: 50, 61; 5: 82; 6-7: 32

Dactylium dendroides 18(suppl.): 19.

Daedalea 1: 7; 5: 86 .

- cinerea 6-7: 31 .

- confragosa 3: 68 .

- mollis 5: 91.

- quercina $2: 49 ; 3: 51 ; 6-7: 35,43,72 ; 8: 10,16 ; 10: 179$, 181,$182 ; 11: 30,31 ; 12: 47,51 ; 13: 45,46 ; 17: 36$.

- unicolor $3: 42 ; 8: 15$.

Daedalopsis confragosa 17: 33, 36, 37.

- $\mathrm{f}$. rubescens 8: 16 .

_ - var. tricolor 5: 91; 10: 181.

Daldinia concentrica 17: 71 .

- vernicosa 17: 71 .

Darluca filum 17: 10, 14.

Dasyscypha 2: 39-41.

- abietis 2: 41 .

- calyciformis 4: 43; 5: 97.

- calycina 2: 41; 11: 41 .

- fuscosanguinea 2: 39-41.

- pini 2: 39-41.

- subtilissima 2: 41 .

- willkommii 11: 41 .

Deconica 5: 67,68; 6-7: 25 .

— atrorufa 3: 40, 49, 59; 4: 38, 56; 5: 68 .

- bullacea 3: 40, 49; 4: 38, 56; 5: 68 .

— crobula 4: $37,38,55 ; 5: 64,67 ; 6-7: 25$.

- inquilina 4: $37,38,55 ; 5: 67$.

- physaloides $4: 61 ; 5: 68$.

- rhombispora $3: 59 ; 4: 37,38,56 ; 5: 68 ; 6-7: 25$.

Deightoniella 15: 42 .

- arundinacea 15: 38, 42, 43.

Delicatula 17: 68 .

— ignobilis 5: 29.

- integrella 4: 31; 5: 25, 30 .

- tenax 4: 38.

Dentinum 17: 69 .

Dermatea eucrita 5: 97.

Dermocybe 2: $56 ; 14: 51$.

— anomala 6-7: 70.

- anthracina 5: 51 .

- cinnamomea $2: 55 ; 3: 22,27,31,37 ; 5: 50,51 ; 17: 63$, 18(suppl.): 41.

- - var. cinnamomeofulva 4: 51

- - var. conformis 5: 51; 6-7: 19 .

_ — f. crocea $4: 36 ; 5: 51$.

_ - f. croceifolia $3: 57,66 ; 4: 36,42,51 ; 5: 50$.

— - var. lutescens $3: 47,57 ; 4: 36: 5: 51$.

— - var. paludosa 3: 57; 4: 52; 5: 52 .

- cinnamomeobadia 4: 51; 5: 50; 6-7: 19.

- cinnamomeofulva 5: 51; 6-7: 19.

- cinnamomeolutea 14: 49, 50, 52.

- cinnamomeolutescens 5: 50; 6-7: 19.

- concinna $3: 57 ; 5: 50 ; 6-7: 19$

- crocea 3: 22, 27, 31, 37, 47 .

- croceocona 5: 50 .

- malicoria 3: 22, 27, 37, 47: 5: 51; 6-7: 19.

- miltina 3: 27, 37, 57, 66; 5: 51 .

—orellana 6-7: 19; 18(suppl.): 7.

— phoenicea $3: 27,37: 4: 51: 5: 51$.

— sanguinea $2: 55 ; 3: 27,37,47,57,66: 4: 51: 5: 51 ; 14: 50$, $51 ; 18$ (suppl.): 41 .

- semisanguinea $2: 55: 3: 22,27,37,47,57: 4: 51 ; 5: 51: 6-7$ : 
19; 14: 50, 51 .

- uliginosa 5: 50.

Desmazierella 12: 61 .

- acicola 12: 61, 62 .

Diachea leucopodia 14: 63, 79; 19: 10.

- - var. globosa 14: 63 .

Dichomitus 20: 2 .

- campestris 18: 46.

Dictydium 14: 79 .

— cancellatum 14: 70, 71, 79: 19: 10 .

_ - var. alpinum 14: 54 .

- cernuum 14: 54; 19: 10.

- microcarpum 19: 10.

— umbilicatum 19: 10.

Dictyophora duplicata 6-7: 109.

Diderma 14: 60 .

— alpinum 19: 3 .

— arboreum 14: 62 .

- chondrioderma 14: 62, 78; 19: 10.

- crustaceum 14: 62, 78; 19: 10.

- effusum 19: 1, 3, 11 .

— globosum 14: 62; 18: 59; 19: 11.

— hemisphaericum 14: 62; 19: 3, 10, 11 .

- montanum 19: 1, 3, 11 .

- niveum 19: 1, 3, 11 .

— radiatum 14: 62; 19: 10-12, 14.

- simplex 19: 8, 11, 14.

- spumarioides 14: 62; 19: 11.

- stellare 19: 11.

- testaceum 14: 62; 19: 10, 11.

- umbilicatum 19: 11 .

Didymium 14: 62; 18: 60; 19: 5 .

- anellus 19: 1, 3, 11 .

- anomalum 18: 60 .

- clavus 14: 63; 18: 60; 19: 11 .

- comatum 18: 58, 59; 19: 11.

- complanatum 14: 63; 19: 11.

- crustaceum 14: 64; 19: 4, 5, 11, 14, 15.

— - var. obducens 19: 3, 11 .

— difforme 14: 62; 18: 58-60; 19: 10, 11, 14, 15.

— dubium 18: 58, 60, 61; 19: 11 .

- effusum 19: 11 .

- farinaceum 19: 11.

- fulvum 19: 1, 3-5, 15.

- hemisphaericum 19: 11 .

— iridis 18: 58,$60 ; 19: 1$.

- lobatum 19: 11.

- melanospermum 6-7: 110; 14: 63, 79; 19: 9, 11, 15.

_- var. minus 14: 63,$64 ; 19: 11$.

- microcarpon 19: 11.

- minus 19: 11 .

— nigripes 14: 64; 19: 11 .

- obducens 1: 1, 3-5, 11, 15.

- physaroides 19: 11 .

- serpula 19: 11.

- squamulosum 14: $64 ; 18: 58,60 ; 19: 11$.

- sturgisii 18: 60 .

Dilophia graminis 11: 6, 16 .

Dilophospora alopecuri 11: 12 .

- graminis 11: 6 .

Discina 9: 9-11; 13: 48-56; 15: 33, 34; 16: 6, 7; 19: 46, 48 .

- accumbens 15: 30 .

- ancilis 3: 53:5: 96.

— apiculatula 13: 51-56; 16: 7.

- convoluta 15: 33 .

— gigas 9: 10.

— korfii 13: 51, 54, 56.

- larryi 15: 30.
- leucoxantha 9: 11; 15: 33.

- macrospora 13: $54,56$.

- melaleuca 13: 51-54; 15: 33, 34.

- olympiana 13: 51-56; 16: 7 .

— perlata 9: 11; 16: 7 .

- - var. geogena (-ius) 15: 30.

- warnei 13: 54.

— venosa 3: 53; 4: 28; 5: 96.

Disciotis 14: 124.

Discosia 15: 44.

- artocreas 15: 38, 43, 44 .

Ditiola radicata $4: 58 ; 5: 82$

Dochmiopus pubescens 4: 49; 5: 39.

Dothidea gangreana 15: 59 .

Drechslera 12: 5 .

— dactylidis 12: 5, 21, 23, 33 .

— dictyoides 12: 8 .

— phlei 12: 17.

- siccans 12: 19.

- tritici-repentis 12: 24 .

- vagans 12: 23.

Drosella 5: 38 .

- fracida 5: 36

—irrorata 4: 49; 5: 36, 39.

Dryodon 1: 35.

— cirrhatum 3: 24, 42, 51; 5: 87; 6-7: 30.

- coralloides 5: 87 .

- corrugatum 6-7: 30.

- diversidens $3: 32,67 ; 4: 40 ; 5: 87 ; 6-7: 30$.

Eccilia 1: 30, 42; 2: 56; 5: 32.

- cretatus 5: 30 .

- lanica 17: 59, 63.

— polita 5: 30 .

- sericeonitida 17: 63 .

— undata 17: 63.

- - var. pusilla 17: 63 .

Echinostelium 17: 31.

- cribrarioides 17: 19-21; 19: 11.

- minutum 17: 19-22, 29-31; 18: 60; 19: 11.

Elaphomyces 6-7: 34.

- cervinus 4: 28; $5: 98$.

- muricatus 5: 98 .

- variegatus 5: 98 .

Encoelia 5: 97 .

Endomyces 9: 46.

Endophyllum pampeanum 17: 6 .

Enerthenema 17: 31.

- papillatum 14: 67; 17: 19, 25, 29, 31; 18: 60; 19: 11 .

Enteridium 14: 72; 19: 15.

— intermedium 19: 11, 13.

- lycoperdon 19: 11, 13.

- olivaceum 14: 71; 19: 11, 13, 15.

— simillimum 19: 11, 15 .

- simulans 19: 11, 15.

- splendens var. juranum 19: 1, 5 .

Entoloma 1: 30, 42; 2: 56.

- aprile 4: 28.

- clypeatum 1: 30 .

- jubatum 1: 30.

- lividum 1: 23, 24, 30, 36, 39.

- majale 1: 30 .

- nidorosum 1: 36, 46.

- porphyrophaeum 1: 30 .

— prunuloides 1: 30.

- sericeum 17: 63.

Entyloma calendulae 17: 6 .

- chrysosplenii 6-7: 95.

Epichloe 15: 49. 
- typhina 15: 46, 49-51

Epidermophyton 1: 48, 50.

— inquinale 1: $48,50,52,55$.

- interdigitale 1: 48 .

Erysiphe aquilegiae 4: 15.

- asperifoliorum 8: 6 .

- artemisiae 4: 15 .

— cichoracearum 4: 16, 21; 6-7: 98; 8: 6 .

- communis 4: 16, 21.

- depressa 4: 16.

— fischeri 4: 16; 6-7: 98 .

— galeopsidis 4: 16: 8: 5 .

— galii 4: 17; 17: 13 .

— framinis $4: 17 ; 6-7: 98 ; 8: 6,7 ; 17: 7,13$.

- horridula 4: 17.

- hyperici 4: 17.

- labiatarum 4: 17.

- lamprocarpa 4: 17.

- martii 2: 46, 47: 6-7: 98; 17: 13.

- montagnei 4: 18; 6-7: 98 .

— nitida 4: 18; 6-7: 99

— pisi 4: 18; 17: 13 .

- polygoni 4: 19.

- polyphaga 4: 19.

- ranunculi $8: 6,7$.

— trifolii 8: 6: 17: 13.

- ulmariae 4: 19 .

- umbelliferarum 4: 19.

- urticae 4: 19.

— valerianae 4: 19.

— verbasci 2: 47; 4: 20.

- vernalis 4: 14, 20.

Escherichia coli 18(suppl.): 6, 7.

Eupropolella 14: 97.

— arctostaphyli 14: 97.

- vaccinii 14: 97, 99, 100

Exidia cartilaginea 4: 43; 5: 82.

- friesiana 3: 29; 4: 60; 5: 82 .

— glandulosa $3: 29 ; 4: 44 ; 5: 81$.

— pithya 4: 60; 5: 81 .

— repanda 4: 44, 58; 5: 82 .

- saccharina $3: 29 ; 4: 58 ; 5: 82$.

- truncata 6-7: 31.

Exobasidium antarcticum 17: 3, 14, 15.

- vaccinii 5: 82.

Fastigiella 15: 33 .

- caroliniana 15: 34.

Favolus 20: 8.

Fayodia 2: 60; 4: 31, 62; 5: 25; 19: 23.

- bisphaerigera $5: 25 ; 6-7: 11$.

- invita 19: 23.

- lacerata 4: 31 .

- maura $2: 56 ; 3: 26 ; 5: 25$.

- striaepilea 3: 26.

Fibuloporia mollusca 8: 16.

Fistulina 1: 7; 2: 48, 49.

- hepatica $2: 49,50 ; 6-7: 35,42,43,68,72 ; 8: 10,16 ; 9: 36$; 13: $46 ; 17: 33,35$.

Flammula 1: 18,$31 ; 2: 56 ; 3: 36 ; 6-7: 19 ; 10: 181$.

- alnicola 10: 181 .

- carbonaria 5: 100.

- graminis 6-7: 25 .

- inopum 6-7: 25.

- muricella 6-7: 25 .

- penetrans 3: 7, 9; 10: 178, 182.

Flammulaster 17: 64

- ferruginea 17: 64 .
Flammulina velutipes $2: 56 ; 3: 26 ; 4: 31,47 ; 5: 23 ; 10: 7,181$; 11: 30,$31 ; 16: 10,12,13 ; 18$ (suppl.): $17,18,29-31,43,59$, $116,117$.

- - f. sphinx 5: 23.

Flavoscypha 14: 105-107, 125, 138, 139, 141.

- cantharella 14: 105, 107.

- phlebophora 14: 105, 107.

Flocculina 17: 64.

- carpophiloides 5: 48.

Fomes alboluteus 20: 2 .

— annosus $3: 8 ; 8: 16 ; 10: 178 ; 12: 36,38,44,63 ; 14: 5-7,16$, $17,28,29$

- cajanderi 18: 39.

- conchatus 5: 92.

- connatus 5: 90 .

- fomentarius $8: 16 ; 10: 181 ; 11: 25,26,31 ; 12: 47-50$, 62,$63 ; 13: 6,44-46 ; 17: 37$.

- igniarius 8: 18; 11: 23.

- pinicola 5: 91 .

— pomacus 17: 77.

- - f. crataegi 17: 77, 80, 84.

- prunicola 17: 77, 80 .

- robustus $2: 49 ; 6-7: 72$

- salicinus 3: 53.

- ungulatus 1: 61, 68-70; 3: 8, 24; 5: 91 .

Fomitopsis 3: 24 .

— annosa 8: 16; 10: 182; 11: 23, 26, 29-31; 12: 47-50; 13: 45

— cajanderi 18: 39.

- connata 5: 90 .

- odoratissima 10: 181.

- pinicola 8: 16; 10: 178, 181, 182; 11: 25, 26, 29-31; 12: 47-51, 61; 13: 45, 46; 17: 37; 20: 6, 7, 10, 12, 13.

- rosea 10: 182; 11: 29, 31; 20:6, 7 .

Fuligo cinerea 19: 1, 5, 11 .

- - var. ecorticata 14: 60, 61 .

— intermedia 14: 60, 78; 19: 11 .

- muscorum 14: 61; 19: 11, 13, 15.

- septica 6-7: 110; 14: $51,52,58,60,61,79 ; 17: 31 ; 18$ : 26; 19: 8-11.

- - var. candida 19: 11 .

- - var. flava 19: 11.

- - var, rufa 19: 11 .

- - var. violacea 19: 11.

- simulans 19: 11, 15.

- varians 19: 11 .

- - var. ecorticata (-um) 19: 11.

Funalia gallica 16: 3

Fusarium 1: 56, 59; 9: 46; 12: 59, 61; 18(suppl.): 51 .

- lycopersici 12: 55.

- roseum 12: 55.

Fusoma triseptatum 15: 59

Gaeumannomyces graminis 19: 54,55 .

Galera 1: 31; 2: 56 .

- hypnorum 3: 7 .

— ravida 3: 48; 5: 64 .

- spartea 3: 48 .

- viscosa 17: 61

Galerina 2: 56, 60, 61; 4: 51; 5: 50; 10: 21, 32; 14: 49, 51, 52.

— ambigua $4: 35,51 ; 5: 49 ; 6-7: 18$.

- badipes $3: 27 ; 5: 49$.

- camerina 6-7: 18.

- clavata 3: 65; 4: 51; 5: 49.

- confragosa 4: 51; 5: 49; 6-7: 18 .

- gibbosa 4: 35; $5: 48$.

— graminea 4: 51; 5: 49. 
- hypnorum $2: 56 ; 3: 27,31,37,47,57,65 ; 4: 35,51 ; 5$ : 48; 6-7: 18; 14: 52 .

- marginata $2: 56 ; 3: 27,37,47,54,57,65 ; 4: 35,51 ; 5$ : $49 ; 6-7: 18 ; 14: 51,52 ; 17: 63$.

- mniophila $4: 42,51 ; 5: 49$.

- mycenoides 4: 35, 51; 5: 48.

- mycenopsis $3: 47,57 ; 4: 35 ; 5: 49 ; 6-7: 18$.

— nana $3: 65 ; 4: 51 ; 5: 49$.

— paludosa $2: 37,56,57 ; 4: 35,51 ; 5: 48 ; 10: 180 ; 14: 52$.

_ pumila $4: 42 ; 5: 49 ; 6-7: 18$.

- - var. subferruginea $4: 35 ; 5: 49 ; 6-7: 18$.

- rubiginosa 5: 48 .

- sahleri 6-7: 18 .

— sideroides $2: 56 ; 4: 51 ; 5: 49$

— sphagnorum 2: 56; 3: 22. 37; 4: 35, 51; 5: 48; 6-7: 18.

- stagnina $4: 51 ; 5: 48 ; 14: 52$.

- tenuissima 3: 47 .

- tibiicystis $3: 57,65 ; 4: 35,51 ; 5 ; 48 ; 6-7: 18$.

— triscopa 3: 57; 4: 35, 51; 5: 49; 6-7: 18.

- uncialis $4: 51 ; 5: 49 ; 6-7: 18$.

- unicolor 3: 54; $4: 35,51 ; 5: 49 ; 6-7: 18 ; 17: 63$.

Ganoderma 3: 51, 68; 5: 91; 6-7: 31; 13: 6 .

- adspersum 17: 35 .

- applanatum $3: 24,29,32,42,51,61 ; 4: 41,59 ; 5: 88$, 91: 6-7: 31; 8: 16; 10: 181; 13: 45; 17: 35 .

- lucidum 3: 51; 5: 91; 6-7: 42; 10: 181; 17: 35.

- pfeifferi 6-7: 42 .

- resinaceum 5: 91 .

Geastrum (Geaster) 1: 79-82.

- argenteus 1: 79.

- bryantii $1: 77,80-82 ; 3: 61 ; 4: 44 ; 5: 94$.

- calyculatus 1: 81, 82.

- coronatum 3: 50; 4: 28; 5: 94.

- floriformis 1: 77-82.

- limbatum 1: 81.

- nanum 19: 19, 20.

- pectinatum $1: 81,82 ; 3: 50,53 ; 4: 44 ; 5: 94 ; 6-7: 32$.

— quadrifidum 19: 19.

- rufescens 1: 81 .

- striatum 3: 50 .

Geophylla 5: 44-47.

Geopora 14: 125.

Geopyxis 14: 125.

— carbonaria 5: 96, 100; 14: 125.

- cupularis $4: 41 ; 5: 96$.

- pustulata 4: 41; 5: 96.

- verruculosa 18: 57; 19: 44

Geotrichum 15: 5, 6, 9-11.

- candidum 15: 5-11.

- matalense 15: 8.

- septicaemium 15: 6 .

Gerronema 10: 10; 17: 67.

- albidum 15: 21, 27; 18: 29, 57.

Gloeocystidium alutaceum 5: 85 .

- - f. citrinum 5: 85 .

- karstenii 5: 84.

- ochraceum 5: 84 .

- praetermissum 5: 84 .

- roseocremeum 5: 85 .

Gloeophyllum abietinum 10: 182; 17: 36.

- sepiarium 8: 16; 10: 178, 182; 11: 29, 31; 12: 47-51; 13: $43,45,46$.

Gloeoporus 13: 6 .

- amorphus 8: 17 .

- dichrous 10: 181; 12: 47-50.

Glomerella cingulata 14: 5 .

Godronia 14: 33, 34, 38, 40, 41, 43.
- cassandrae 14: 43.

- - f. betulicola 14: 33,43 .

- multispora 14: 33-44.

- urceolus 14: 33, 43 .

Gomphidius 1: 32, 44; 10: 171.

- glutinosus 1: 21,87,88; 2: 56; 3: 7, 8, 19, 25, 30,33, 43,$54 ; 4: 29,45 ; 5: 8,9,14: 50 ; 18$ (suppl.): 41, 114, 116 , 117.

- maculatus 18(suppl.): 41 .

— roseus $2: 56 ; 3: 19,25,43 ; 4: 29 ; 5: 8 ; 6-7: 6$.

— rutilus $2: 56 ; 3: 19 ; 5: 9 ; 14: 49,50,52$.

- subroseus $4: 45 ; 5: 8$

— viscidus $2: 56 ; 3: 7,12,14,19,25,33,43 ; 4: 29,45 ; 5$ : 9; 6-7: 37.

Gomphus clavatus 5: 81 .

Grandinia 6-7: 29 .

- brinkmannii 5: 86 .

- deflectens 5: 84

- fugax 6-7: 29.

- raduloides 4: 44; 5: 86

Grifola frondosa 6-7: 42.

- umbellata 6-7: 42 .

Gymnoascus 15: 10.

Gymnomitrula 9: 23, 29.

- abietis 9: 23 .

— gracilis 9: $23,27,29$.

- rehmii 9: 27, 29.

Gymnopilus 2: 56; 3: 57; 5: 48; 6-7: 18, 19.

- flavus 6-7: 18

- fulgens 6-7: 19.

- hybridus $3: 22,27,37,57,65 ; 4: 36,51 ; 5: 50$.

- limulatus 4: 35,51 .

- liquiritiae $3: 22,27,47,57 ; 5: 50$.

- microsporus 6-7: 18.

- penetrans $2: 56 ; 3: 22,37,47,57 ; 4: 36 ; 5: 50 ; 6-7: 18$; $11: 26,29,31 ; 12: 47-49 ; 14: 51 ; 18$ (suppl.): 41 .

— picreus 2: 56: 3:22, 27, 47; 4: 51: 5: 50; 18(suppl.): 41 .

- sapineus $3: 22,27,31,37 ; 4: 35,51 ; 5: 50$.

- - f. sapineus 3: 22 .

- spectabilis var. junonius $3: 57 ; 5: 50$.

Gyrocephalus aginnensis 19: 47.

Gyrodon 2: 56.

- labyrinthicus 6-7: 53 .

- lividus 2: 53; 5: 8; 6-7: 53 .

- sistotrema 6-7: 53 .

Gyromitra 1: 23; 4: 51; 5: 95; 6-7: 34; 9: 9-11, 13, 19; 10: 41; 13: 48-56; 14: 110,111, 125, 139-141; 15: 29, 33, 34, 36,$37 ; 16: 6,7 ; 18 ; 57 ; 18$ (suppl.): 6,$58 ; 19: 43,46,48,49$, 116,117 .

- accumbens 15: 29, 30.

— ambiqua 9: 10, 13-19; 13: 51-56; 14: 111, 125; 15: 29, 36,$37 ; 16: 6,7,9$.

— apiculatula 13: $48,56,57$.

- bubacii 19: 47.

- californica 9: 9, 10; 13: 48, 49, 54.

- caroliniana 13: 52, 56; 15: 33 .

- subg. Discina 13: 48

- esculenta 1: 15, 16, 23-25, 36, 39, 45, 88; 3: 24, 30, 32 , 42,$52 ; 4: 41 ; 5: 95 ; 6-7: 34 ; 8: 37,48,56,59 ; 9: 10 ; 13$ : $51-56 ; 15: 29,34,36,37 ; 16: 1-3,6,7,9,10,12,13$; 18(suppl.): 5, 6, 29-31, 39, 43-45, 56, 58-62, 107, 114, 115, 117: 19: 46-48.

- - var. alba 19: 46.

- - var. aurantiaca 19: 46 .

— fastigiata 13: 51,$56 ; 16: 7$.

- gabretae 9: 9, 10.

- geogenia 15: 29, 30 . 
_ gigas 1: 24, 25, 36; 3: 53; 4: 28; 5: 95; 6-7: 34; 9: 10, 11 , 14: 13: 48, 51-57; 15: 29, 37; 16: 6, 7, 9; 18(suppl.): 58 .

— inflata 19: 47.

— infula $3: 30,42,68 ; 4: 59 ; 5: 95$ 9: $10,11,13-19 ; 13: 51-56$; 15: $29,36,37 ; 16: 6,7,9 ; 19: 46$.

_- var. apiculatispora 9: 10, 17, 19.

- f. gyrosa 9: 13 .

— intermedia 15: 29, 30.

- korfii 13: 48, 56, 57; 15: 29.

- krombholzii 19: 47.

- labyrinthica 13: 56 .

- larryi 15: 29, 30.

- leucoxantha 9: 10, 11; 13: 54, 55.

- macrospora 13: 48, 56 .

- melaleuca 15: 29, 33, 34; 19: 49.

- montana 13: 48, 51, 56, 57; 15: 29; 16:6, 7, 9 .

- neuwirthii 19: 47.

- olympiana 13: $48,56,57$.

— perlata 9: 10,$11 ; 13: 51,54,56 ; 15: 29,37$.

- queletii 19: 47 .

- recurva 18: 57; 19: 46, 48, 49.

- sphaerospora 9: 10, 11 .

- splendida 19: 46, 47 .

- suspecta 1: 36 .

— tasmanica 9: 11; 13: 51, 52, 54, 55: 15: 29; 16: 7.

- warnei 13: 48, 56.

Gyromitroides 14: 110

Gyrophana lacrymans 5: 86.

- pinastri 5: 86 .

Gyroporus 2: 56.

- castaneus 6-7: 53, 56, 73; 8: 11; 18(suppl.): 6 .

— cyanescens $2: 53 ; 5: 8 ; 6-7: 52,68,71 ; 8: 11 ; 18$ (suppl.): 6 .

Hadrotrichum 15: 41.

- microsporum var. macrosporum 15: 41.

- virescens 15: 38, 40-42.

Hapalopilus alboluteus 11: 33 .

- nidulans 8: 17; 10: 181

- ochraceolatertius 8: 17.

Hebeloma 1: $31: 43 ; 3: 46,59 ; 5: 65 ; 6-7: 23,24: 14: 10$. 11.50 ; 18(suppl.): $41,93$.

- birrum 6-7: 15 .

- claviceps $4: 34,49 ; 5: 41$.

- crassum 3: 58:5:59.

- crustuliniforme $1: 25,31,43: 2: 56 ; 3: 21,27,36,46,56 ; 4$ : 34. 49: $5: 41: 10: 174$

- denudatum 4: 34 .

-- edurum 5: 41 .

- elatum 3: $21 ; 5: 41$.

- fastibile $3: 35,46,56: 4: 49: 5: 40$

- firmum 4: 34, 49; 5: 41; 6-7: 16.

- hiemale $4: 34,49 ; 5: 41$.

- Iongicaudum $3: 21,27,31,36,46 ; 5: 41: 6-7: 16 ; 14: 10$.

— mesophaeum 2: 56; 3: 35, 46, 65; $4: 34,49 ; 5: 40 ; 6-7: 15$.

- nudipes 4: 49:5: 41 .

- porphyrosporum 6-7: 15

- pumilum 3: 46, 65: 4: 34, 49: 5: 41:6-7: 16.

- punctatum $3: 46 ; 5: 41$.

_pusillum $3: 46,56 ; 4: 34: 5: 41$.

— sacchariolens $3: 21 ; 4: 48 ; 5: 30,41: 6-7: 16 ; 15: 27$

- sarcophyllum 6-7: 15

- sinapizans $3: 21 ; 4: 49 ; 5: 41$

- spoliatum 4: 34; $5: 41$.

_- strophosum $3: 35,65 ; 4: 34,49 ; 5: 40 ; 6-7: 15$.

- subsaponaceum 3:21, 36:5: 41.

- testaceum 3: 35, 46. 56: 4: 34, 49: 5: 41: 6-7: 15, 16.

- iruncatum 3: 36. 65: 4: 49:5: 41 .

- versipelle $4: 49 ; 5: 40 ; 6-7: 15$.
Helminthosporium 12: 5, 6, 14, 32; 13: 27.

- acrothecioides 12: 26.

- arundinaceum 15: 42.

- avenae 12: 6

- biforme 12: 5, 10, 13-15, 21, 27, 28, 30, 33.

- biseptatum 12: 27.

- catenarium 12: 14, 15, 22.

- dactylidis 12: 10, 14, 20, 30 .

- dictyoides 12: 8, 14, 19.

- - f. sp. dictyoides 12: 5, 8-15, 17, 22, 29, 32

- f. sp. perenne 12: 5, 8-10, 12, 15-17, 22, 29, 32.

- var. phlei 12: 17

- flexuosum 12: 6 .

- gramineum 12: 6 .

- phlei 12: 5, 9, 10,12, 14, 17-19, 30, 32

- poae 12: 23 .

- sativum 12: $5,10,14,15,21,25,26,30,33,55$.

- siccans 12: 5, 9, 10,14,15, 17, 19-22, 29, 32.

- sorokianum 12: 26.

- triseptatum 12: $5,10,14,21,28,30-33$.

- tritici-repentis 12: 5, 9, 10, 21, 24-26, 30, 32, 33.

— vagans 12: 5, 9, 10,14,18,21, 23, 24, 30, 32.

Helotium 5: 97: 6-7: 34; 14: 51 .

— citrinum 4: 59; $5: 98$.

- conscriptum 5: 97.

- praecox 5: 98 .

- scutula 4: 43; 5: 97

Helvella 1: 36,$39 ; 6-7: 67 ; 9: 9,11 ; 13: 48,49,51,54 ; 14$ 102-104, 106, 110,111, 125, 139; 15: 29, 30, 34; 16: 7; 17: $40-42,44-46,49,50,54,55,75 ; 18: 57 ; 18$ (suppl.): $6 ; 19$ : $33,38,43$.

— acetabulum 13: 50, 55; 14: 103, 106, 110, 116; 15: 27. 30: 17: 45-58; 19: 33, 38-40, 42, 44

- albipes 6-7: 34 .

- ambigua 9: 13, 17.

— arctoalpina 17: 45-58; 19: 38, 44.

— atra $3: 24 ; 5: 95 ; 14: 116$.

— aurantiaca 9: 24

- beatonii 14: 102, 103.

- bicolor 6-7: 42 .

- brevis 14: 102, 104 .

- californica 13: 49, 56; 14: 110, 112 .

- confusa 17: 40-44: 19: 33, 36, 37, 40, 42 .

- corium 17: 52 .

- costifera 15: 21, 27: 17: 40, 42, 46, 53:18:57;19:33-35. $38-40,42$.

- crassitunicata 15: 30; 17: 42 .

- crispa 6-7: 46, 67, 68:8:9, 23; 14: 106 .

- cupuliformis 17: 20, 42 .

- dryadrophila 17: 32, 45-58: 19: 33, 38, 43, 44.

- elastica 6-7: 67, 68: 14: 116.

- erythrophea 19: 47

- esculenta 19: 47

— friesiana 9: 13 .

- helvellula 17: 42 .

- hyperborea 18: 29, 57: 19: 33-35, 37-40, 43.

- infula 19: 13,16.

- - var. elongata 9: 17.

— - f. friesiana 9: 13 .

- - var. similis 9: 17.

- lacunosa $3: 24: 5: 95 ; 6-7: 34 ; 13: 55 ; 14: 103 ; 15: 27 ; 17$ : 46.

- laricina 9: 24.

- leucomelaena 3: 54: 14: 102, 103: 15: 30: 17: 40-44: 18:57; $19: 33,36,37,40,42$.

- f. otideata 14: 103.

- macropus 13: 54: 14: 102. 104: 15:22: 17: 42. 
- - var. brevis 14: 102, 104.

- oblongispora 18: 57; 19: 33, 36, 37, 40-42.

— palustris 17: 42, 45, 46; 18: 29; 19: 36-38.

— pedunculata 18: $57 ; 19: 33,40-42$.

- pezizoides 15: 28; 19: 28 .

- pocillum 15: 29, 30; 17: 45, 46, 49-51, 53, 55.

- queletii 17: 40-42, 46; 19: 33, 40, 43, 44.

— - var. alpina 19: 43 .

- rhodopus 9: 13.

- silvicola 14: 102, 103; 17: 42, 46.

- sinuosa 19: 47.

- solitaria 17: 40-44, 46; 19: 33, 37, 40, 42-44.

— sphaerospora 9: 9; 13:49; 14: 110.

— suspecta 1: $36 ; 19: 47$.

$\rightarrow$ ulvinenii 19: 33, 41-43.

- verruculosa 18: 57; 19: 33, 44.

Helvellella 14: 110.

Hemiarcyria calvata 19: 11 .

— karstyenii 19: 11, 12, 15 .

- rubiformis 19: 11, 12 .

Hemileia 13: 38.

Hemitrichia 14: 75; 17: 31; 19: 15.

— abietina 14: 75; 19: 11, 15.

- clavata 14: 75: 19: 11, 13.

- karstenii 14: 76; 19: 11, 15 .

- serpula 14: 76; 19: 12 .

- vesparium 14: 75; 19: 12

Hendersonia crastophila 19: 54, 57.

— graminis 19: 54 .

Heterobasidion annosum 18: 37; 20: 19.

Heterodictyon mirabile 14: 54 .

Hexajuga giovanellae 4: 32 .

Heydenia 14: 133, 135.

— alpina 14: 134, 135.

- americana 14: 134-136.

Heyderia 9: 23, 29, 30.

- abietis 9: 124, 27, 28, 32, 33; 15: 28.

- - var. abietis 9: 33 .

— - var. pusilla 9: 33 .

Hirschioporus abietinus 8: 17; 10: 182; 11: 26, 31; 12: 47-51; 13: $45: 17: 37$

- fuscoviolaceus 8: 17.

- pargamenus 17: 37 .

Hohenbuehelia 2: 62 .

- geogenia 3: 45; $5: 23$.

- - var. queletii 5: 23.

- petaloides $3: 45 ; 6-7: 10$.

- reniformis 6-7: 10 .

- semiinfundibuliformis 5: 23.

— serotina $2: 57 ; 3: 26 ; 4: 47 ; 5: 23 ; 10: 181,182 ; 12: 63$.

— - var. flaccida 5: 23 .

Homostegia gangraena 15: 56 .

Hormoconis 14: 133, 135.

Hormodendron 1: 56-59.

- resinae 14: 135 .

Humaria 5: 96; 14: 125; 19: 52.

— fusipora 4: 59; 5: 96.

- hemisphaerica 15: 28; 19: 52.

- rhodoleuca 17: 74, 75.

Humicola 18 (suppl.): 74, 75.

Hyalopsora cheilanthis 17: 6 .

Hydnellum 6-7: 30, 31.

- aurantiacum 6-7: 30 .

- caeruleum 6-7: 30 .

- compactum 6-7: 30.

- diabolus 6-7: 30

- ferrugineum 6-7: 30; 14: 51, 98.
- suaveolens 6-7: 30.

— sulphureum 6-7: 30.

— velutinum var. queletii 6-7: 31 .

- - var. scrobiculatum 6-7: 31.

- - var. zonatum 6-7: 31 .

Hydnum 1: 10, 35: 3: 8, 16; 18(suppl.): 5.

- aurantiacum 3: 7 .

- cirrhatum 2: 49.

- fulgens 20: $8,10$.

— imbricatum 3: 7; 18(suppl.): 7.

- repandum $3: 7,24,29,32,42,51,61 ; 4: 42,60 ; 5: 87 ; 8: 46$. 48,$59 ; 17: 69 ; 18$ (suppl.): $6,41,108,115,117$.

- rufescens $3: 7,8,24,29,32,42,51,61 ; 5: 87 ; 8: 46,48,59$; 17: 69; 18(suppl.): 18, 29, 31, 81, 83, 115, 117 .

- septentionale 10: 181 .

- sordidum 5: 86.

Hydrocybe 2: 57; 3: 39, 48; 4: 60; 5: 51, 52, 57; 6-7: 20.

— acuta 3: 28, 31, 39, 48, 58, 66; 4: 36, 43, 54, 60; 5: 58 .

- - var. striatula $3: 66 ; 5: 58$.

— adalbertii 3: 66; 4: 52; 5: 55; 6-7: 21, 22.

— alnetorum $3: 66 ; 4: 36,53 ; 5: 56 ; 6-7: 20$.

- angulosa $4: 36 ; 5: 56 ; 6-7: 21$.

— armeniaca 2: 55; 3: 28, 31, 38, 47, 58, 66; 4: 36, 52:5: 53

— armillata 2: 55; 3: 22, 27, 31, 37, 47, 57; 4: 36, 51; $5: 51$.

- atrocoerulea 6-7: 22.

- balaustina $3: 38,58 ; 4: 36,43,53 ; 5: 56 ; 6-7: 21$.

- betulorum 4: 52; 5: 52; 6-7: 19.

— bicolor 3: 47, 58; 5: 54; 6-7: 20

— biformis $3: 28,38,47,66 ; 4: 52 ; 5: 53 ; 6-7: 20$.

— bivela $2: 55 ; 3: 22,28,47 ; 4: 52 ; 5: 53 ; 6-7: 20$.

— biveloides 4: 52; 5: 53; 6-7: 20 .

— bovina $3: 38,58,66 ; 4: 36,52 ; 5: 52,55 ; 6-7: 19$.

- brunnea $2: 55 ; 3: 22,28,31,38,48,58 ; 4: 36,52 ; 5: 55 ; 6-7$ : 19.

- brunneofulva $3: 28,48,58,66 ; 4: 36,52 ; 5: 55 ; 6-7: 21$.

_ bulbosa $3: 37,47,66 ; 4: 36,43,52 ; 5: 52 ; 6-7: 20$.

— bulliardii 5: 51 .

- callistea f. limonia 5: 52 .

— candelaris 3: 28, 31, 38, 66; 5: 53.

— castanea $2: 55 ; 3: 31,47,58,66 ; 4: 36,52 ; 5: 54 ; 6-7: 20$.

- cedriolens 6-7: 22.

— colus $3: 47 ; 5: 51$

— crassifolia 4: 53; 5: 56 .

— cypriaca $3: 58 ; 5: 54 ; 6-7: 20$

—damascena 3: 28, 38, 58, 66; 4: 52; 5: 53; 6-7: 20.

— decipiens 3: 22, 38, 39, 48, 58; 4: 54; 5: 55, 58; 6-7: 22.

- - var. rickeniana 6-7: 22.

— diluta $3: 31,38,47 ; 4: 36 ; 5: 53$.

— divulgata 6-7: 20 .

- duracina $3: 38,48,66 ; 4: 52 ; 5: 54 ; 6-7: 20$

— - var. raphanica $3: 66 ; 5: 53 ; 6-7: 20$.

- erugata 6-7: 20.

- erythrina $3: 22,37,47,58,66 ; 4: 36,43,52 ; 5: 54,55,58$ : 6-7: 21 .

— evernia 2: 55; 3: 22, 58; 4: 36, 52; 5: 54; 6-7: $20,22$.

— fasciata $3: 22,28,39,48,58 ; 4: 54 ; 5: 58 ; 6-7: 22$.

- flexipes $2: 55 ; 3: 28,31,38,48,58,66 ; 4: 36,53 ; 5: 57 ; 6-7$ : 22.

- fulvescens 3: 58; 4: 53; 5: 58 .

— gladicolor 3: 28, 38, 48, 58, 66; 4: 52; 5: 55 .

- - var. curta $3: 38 ; 5: 55 ; 6-7: 21$.

- helobia 5: 56; 6-7: 21 .

- helvelloides 3: 47; $5: 52$.

— helveola $3: 38,66 ; 4: 53 ; 5: 55 ; 6-7: 21$.

— hemitricha $2: 55 ; 3: 28,38,48,58,66 ; 4: 36,53 ; 5: 52,57$ : 6-7: 21, 22.

_ - var. americana 6-7: 22. 
- heterospora 6-7: 22 .

- hinnulea $3: 22,28,38,48,66 ; 4: 53 ; 5: 55,56 ; 6-7: 21,22$.

_ - f. robusta 4: 53 .

— hinnulodes 4: 53; 5: 55; 6-7: 21.

- hoeftii 6-7: 20 .

— holophaea $3: 38,48 ; 4: 52 ; 5: 55 ; 6-7: 21$.

- iliopodia 6-7: 21 .

— incisa $2: 55 ; 3: 28,38,48,66 ; 4: 36,53 ; 5: 57 ; 6-7: 22$.

— isabellina 6-7: 19.

— jubarina $3: 58 ; 4: 52,53 ; 5: 53,56 ; 6-7: 21$.

— junghuhnii 3: 22, 39, 48, 58; 5: 58; 6-7: 22 .

- lanata 5: 52 .

- laniger(-a) 2: 55:3: 47, 58; 4: 36, 52; 5: 52, 53; 6-7: 19, 20.

- limonia $3: 37,58 ; 5: 52 ; 6-7: 19$.

- macropus 5: 52, 53; 6-7: 19.

— melleopallens 4: 52; $5: 53$.

— milvina 3: 38; 4: 52; $5: 55$.

- multicolor 6-7: 21 .

- multivaga 5: 56; 6-7: 21 .

- nigricans 5: 57 .

—obtusa $2: 55 ; 3: 28,38,48,58,66: 4: 53,60 ; 5: 57,58 ; 6-7$ : 22.

— paleacea 3: 31, 38, 48, 58, 66; 4: 36, 43, 53; 5: 57; 6-7: 22.

— paludosa 4: 52; $5: 52$.

— paragaudis $3: 57 ; 5: 51$.

- parevernia 3: 58, 66; 5: 54.

- pateriformis 3: 66; 5: 55 .

- privigna 5: 53 .

— privignoides 4: 52; $5: 53$.

- privignorum 6-7: 20.

— psammocephala 4: 52; 5: 52; 6-7: 19.

- pseudocolus 5: 51, 52 .

- pseudoduracina 3: 66; 4: 36, 52; 5: 53 .

- pseudoprivigna 5: 53; 6-7: 20.

- pulchripes $5: 55 ; 6-7: 21$.

- punctata $3: 38,58 ; 5: 57 ; 6-7: 22$.

— rigens $3: 28,38,48,58 ; 4: 52 ; 5: 53 ; 6-7: 20$.

— rigida $3: 28,38,48,58,66 ; 4: 43,53 ; 5: 55,57 ; 6-7: 21,22$.

— rubricosa $3: 28,48,58,66 ; 4: 53 ; 5: 56 ; 6-7: 21$.

- safranopes 4: 53; 5: 56 .

— saniosa 3: 27, 37, 47, 57, 66; 4: 36, 52; 5: 52; 6-7: 19.

— - var. major 6-7: 19.

_ - var. paludosa $3: 27,31,57,66 ; 5: 52 ; 6-7: 19$.

— saturata $3: 38 ; 4: 36 ; 5: 53 ; 6-7: 20$.

-- saturnina 3: 58; 5: 54; 6-7: 20.

— - var. bresadolae 4: 42, 52; 5: 54; 6-7: 20 .

— scandens 3: 28, 38, 48, 58, 66; 4: 36, 53; 5: 57.

- sciophylla $3: 58 ; 5: 54$.

- scutulata $3: 28,58 ; 4: 52 ; 5: 54$.

- sericeofulva 4: 52; 5: 53; 6-7: 20 .

- sertipes $5: 55 ; 6-7: 21$.

- sphaerospora 6-7: 21.

- stemmata 3: 66; 4: 53; 5: 52, 55, 57; 6-7: 22.

— striaepilea $3: 66 ; 4: 36,53 ; 5: 57 ; 6-7: 22$.

- striatula 5: 58; 6-7: 20 .

- subbalaustina $3: 38,48 ; 4: 36,43,53 ; 5: 56$.

- subferruginea 3: $31,47$.

_ - var. imbuta 3: 28, 37, 66; 5: 53.

- subsertipes 5: 55 .

- subviolascens 6-7: 20 .

- suilla 4: 36; 5 : 54 .

— tortuosa $3: 28,37,58 ; 4: 52 ; 5: 54$.

- torva $2: 55 ; 3: 22,28,37,47,58 ; 5: 54 ; 6-7: 20$.

— triformis 3: 22, 37, 47; 4: 52; 5: 53; 6-7: 20.

- - var. melleopallens 5: 53.

- uliginobtusa 5: 58; 6-7: 22.

- umidicola 6-7: 20.
- uracea 3: 22, 38, 48, 58, 66; 4: 53; 5: 56; 6-7: 21.

- urbica 3: 28 .

Hydropus marginellus 4: 31; 5: 25.

Hygrocybe 1: 32, 44; 3: 10, 63; 5: 10, 13.

- amoena 3: 20, 54 .

- cantharella $3: 25,30,33,43.54 .62 ; 5: 11$.

- ceracea $3: 43,62 ; 5: 10$.

— chlorophana $2: 57 ; 3: 33,43,54 ; 5: 10 ; 6-7: 6$.

— citrina 2: 57; 5: 10 .

- - var. glutinipes $3: 33,62 ; 4: 45 ; 5: 10 ; 6-7: 7$.

— coccinea 2: 57; 3: 33, 54, 62; 5: 10.

— conica $1: 44 ; 2: 57 ; 3: 20,25,33,43,54 ; 4: 29,45 ; 5: 10 ; 17$ : 64.

- constans 3: 20, 54; 5: 10 .

- crocea 5: 10; 6-7: 6 .

- fornicata $3: 43 ; 5: 10$.

- irrigata 6-7: 7 .

- laeta $2: 57 ; 3: 7,12,14,62 ; 5: 10 ; 6-7: 7$.

- langei 3: 20, 54; 5: 10; 6-7: 6 .

- marchii 5: 10; 6-7: 7 .

- miniata $2: 57 ; 3: 7,20,25,33,43,54 ; 4: 44,45,60 ; 5: 11$.

- nigrescens 3: 43, 54, 62; 4: 29; 5: 10; 6-7: 6 .

— nitrata $2: 57 ; 3: 7,25,33,43,54,62 ; 4: 45 ; 5: 10$.

- obrussea $3: 20,54 ; 6-7: 6$.

- ovina $3: 33 ; 5: 10$.

— psittacina 2: 57; 3: 33, 54, 62; 5: 10; 6-7: 6 .

- punicea 2: 57; 3: 52, 62; 5: 10; 6-7: 43, 44, 60, 69; 18(suppl.): 116 .

- quieta 5: 10; 6-7: 7 .

- reai var. insipida $3: 25,43,62 ; 4: 45 ; 5: 10$.

- rickenii 5: 10.

- streptopus 5: 10.

— tristis 3: 20, 33; 5: 10.

- - var. nigrescens 3: 20.

— unguinosa $2: 57 ; 6-7: 7$.

- vitellina 5: 10 .

Hygrophoropsis 2: $53 ; 10: 9$.

- albida 5: 12 .

- aurantiaca $2: 19,57 ; 3: 25,33,43,54 ; 4: 29,42,45,60 ; 5$ : 12; 9: 40-42; 10: 180; 18(suppl.): 41 .

- - var. nigripes 5: 12 .

— f. pallida 3: 52; 4: 29; 5: 12 .

- flava 3: 52 .

Hygrophorus 1: 20, 32, 44; 2: 53, 57, 59; 3: 7, 10; 5: 10; 6-7: 6; 10: $7,116,171 ; 15: 25,26$.

— agathosmus $2: 49,57 ; 3: 20,25,33,43 ; 4: 29,45,60 ; 5: 9$; 14: $50 ; 15: 25-27$.

- aureus 6-7: 6 .

— bicolor 2: 57; 3: 19; 5: 9 .

- bresadolae 6-7: 6 .

- camarophyllus 2: 57; 5: 9; 8: 37, 43, 47-49, 51, 57, 59, 60; 15: $17 ; 18$ (suppl.): 115,117

- caprinus 2: 57; 3: 20, 25, 33, 43; 5: 9.

- chrysodon 17: 63 .

- conicus 1: 23, 32 .

- cossus 1: 32; $2: 57 ; 3: 33,43,62 ; 5: 9$.

- dichrous 5: 9; 6-7: 6 .

- discoideus 3: 62; 5: 9 .

- eburneus $2: 57 ; 3: 33,43 ; 5: 9 ; 6-7: 6 ; 10: 116$.

— erubescens $1: 32 ; 2: 57 ; 3: 20,25,33,43 ; 4: 45 ; 5: 9 ; 6-7: 6$.

- fuscoalbus $3: 62 ; 4: 45 ; 5: 9$.

- hyacinthinus 15: 22, 25, 27, 28.

- hypothejus $2: 57 ; 3: 25,62 ; 4: 45,60 ; 5: 9$.

— karstenii 2: 57; 3: 19, 33; 5: 9; 14: 51; 18(suppl.): 116, 117.

- limacinus 4: 45; 5: 9; 6-7: 6 .

- mesotephrus 3: 62; 4: 45; 5: 9; 6-7: 6 .

- monticola 15: 26, 28. 
- nemoreus 5: 9.

- nitratus 1: 32 .

- olivaceoalbus $2: 57 ; 3: 20,33,43,54,62 ; 4: 29,45,60 ; 5: 9$; 6-7: $6 ; 14: 50$.

- - var. gracilis 6-7: 6 .

- pacificus 15: 25, 26, 28.

— piceae 3: 43, 54, 62; 4: 45; $5: 9$.

- poetarum 5: 9; 6-7:6.

- pudorinus $1: 32 ; 3: 20,33,43 ; 5: 9 ; 6-7: 6$.

— pustulatus 2: $57 ; 3: 25,33,62 ; 5: 9$.

- - var. terebratus 5: 9 .

- tephroleucus $3: 33 ; 5: 9$.

- vinicolor 15: 26, 28.

Hymenochaete rubiginosa 5: 86; 10: 181.

- speciosa 6-7: 6 .

- tabacina 5: 86 .

Hypholoma 1: 32, 43, 88; 2: 57; 3: 14; 14: 49.

- candolleanum 3: 7 .

- capnoides $1: 88 ; 2: 48,49 ; 3: 7 ; 9: 40,41 ; 10: 182 ; 11: 26$, 31; $12: 48,49 ; 14: 49-51$.

- cascum 5: 73 .

- cotoneum 5: 73 .

- elongatipes 14: 52 .

- fasciculare 1: $26,32,43 ; 3: 7,9 ; 10: 181 ; 11: 26$.

- myosotis 14: 52.

- pertinax 5: 72 .

- polytrichi 14: 50 .

— sublateritium $2: 48-50 ; 3: 7.9 ; 10: 181 ; 11: 26 ; 13: 45$.

- udum 14: 52.

Hypocrea citrina 4: 43; 5: 98

Hypopteris graminis 15: 47.

Hypoxylon 5: 98.

- deustum 5: 98 .

— fuscum 5: 98; 6-7: 34.

- howeianum 17: 71.

- multiforme 5: 98 .

- sassafras 17: 59, 60, 71.

Incrustoporia nivea 17: 33, 37.

- semipileata 17: 37.

Inocybe 1: $20,24,25,31,39,43 ; 3: 36,65 ; 4: 34,35,50,51$, 55; 5: 20, 34, 41-46; 6-7: 12, 16-18, 24, 26; 10: 169; 11: 38; 17: 63 .

- abietis $4: 50 ; 5: 44,45$.

— abjecta 3: 27, 31, 47, 65; 4: 34, 50; 5: 44.

— acuta $3: 21,36: 4: 50 ; 5: 46$.

— asterospora $3: 21,27,36,47,57 ; 4: 50 ; 5: 46 ; 6-7: 17$.

— auricoma 4: 50; 5: 45 .

- bispora 4: 34 .

- boltonii 3: 21, 36, 47, 57; 4: 35, 50; 5: 46; 6-7: 15 .

- bongardii 4: 47: 5: 19, 41, 42; 6-7: 24 .

- bresadolae 6-7: 18.

- brunnea 4: 34, 50; 5: 45: 6-7: 17.

- caesariata var. fibrillosa $3: 21,36 ; 5: 43$.

- calamistrata 2: 57 .

_ $\mathrm{f}$. gracilis $3: 31 ; 5: 43$.

- calospora 5: 46

- candida 4: 49.

- capucina 5: 47

— carpta 3: 21, 36; 4: 35: 5: 46, 47; 6-7: 16 .

- casimiri 5: 47:6-7: 18

- cervicolor $3: 65 ; 5: 42$.

- cicatricata $4: 50: 5: 47$.

- cincinnata $3: 36,56 ; 4: 49 ; 5: 42: 6-7: 16$.

- clypeus 4: 51 .

- confusa $4: 34: 5: 45 ; 6-7: 16$.

— cookei 3: 21, 27, 36, 47, 57; 5: 43.

- decipiens 3: 65; 5: 46 .
- decipientoides 3: 57; 4: 35, 50, 51; 5: 47; 6-7: 17.

- deglubens 4: 50; 5: 44, 45; 6-7: 16.

- - var. bulbillosa 5: 44 .

- - var. subbulbillosa 4: 50 .

- delecta 3: 21, 36; 5: 43; 6-7: 16 .

— descissa 3: 21, 36: 4: 50; 5: 44.

— - var. auricoma 3: 47,65; 4: 42, 50; $5: 45$.

_- var. brunneoatra $3: 47,57,65 ; 4: 34,50 ; 5: 44$.

- - var. microspora 3: 21; 5: 44.

- dulcamara $3: 27,65 ; 4: 49 ; 5: 43 ; 6-7: 16$

- eutheles $4: 34,35 ; 5: 42,45$.

— fastigiata $1: 31,36,43 ; 2: 57 ; 3: 21,31,36.47,57 ; 4: 34,50$ : 5: $43 ; 6-7: 16 ; 17: 63$.

- - var. brevispora 5: 43; 6-7: 16 .

- - var. curreyi 5: 43 .

- - var. (f.) superba 5: 43; 6-7: 16.

- - var. umbrinella 5: 43 .

— fibrosa var. trivialis $4: 50 ; 5: 46$.

- fibrosioides 6-7: 17 .

- flocculosa 3: 36, 47; 4: 34, 50; 5: 44; 6-7: 16

- friesii f. nemorosa $5: 44 ; 6-7: 16$

- gausapata 4: 34; 5: 44; 6-7: 16.

- geophylla $1: 31,36,43 ; 2: 57 ; 3: 7,21,27,36,46,56 ; 4: 34$. $35 ; 5: 32,42,44-47,60 ; 6-7: 16-18,60,70,72 ; 18$ (suppl.): 41.

— - var. alba 4: 49; $5: 42$

- - var. candida $5: 42$

- - var. lateritia $3: 36 ; 5: 42$.

- - var. lilacina $3: 27,36,46,65 ; 5: 42$.

- - var. reflexa 5: 42 .

- - var. violacea $5: 42$

- globocystis $3: 57 ; 4: 35,51 ; 5: 47$.

- godeyi 6-7: 16; 15: 27.

- grammata $3: 36 ; 4: 50 ; 5: 46 ; 6-7: 17$.

_ - var. minor 6-7: 17 .

- gymnocarpa 17: 59, 63.

— halophila 4: $34 ; 5: 44$.

— hirsuta 4: 34, 49; 5: 43; 6-7: 16.

— hirtella $3: 57 ; 5: 45 ; 6-7: 17$.

- hiulca $3: 36 ; 5: 46 ; 6-7: 17$.

- hypophaea 5: 45 .

- hystrix 5: 44.

- f. minor 3: $21 ; 5: 43$

- incarnata 5: 42.

— inconcinna 4: 50; 5: 44

— jacobi 3: 57; 5: 46 .

- lacera $2: 57 ; 3: 21,27,31,36,47,57 ; 4: 34,50 ; 5: 44,45$ : 6-7: $16,70,111,112$

- $\mathrm{f}$. gracilis $3: 31 ; 5: 44 ; 6-7: 16$.

- - var. halophila 6-7: 111 .

- langei 3: 47, 65; 4: 34; 5: 45; 6-7: 17.

— - f. bispora 4: $35 ; \mathbf{5 : 4 5}$.

- - f. major 6-7: 17.

- lanuginella $3: 21,37,47 ; 4: 50 ; 5: 47 ; 6-7: 17$.

- lanuginosa $2: 57 ; 3: 31,37,47,57 ; 4: 51 ; 5: 47 ; 6-7: 17$.

- - var. casimiri 4: 51 .

- longicystis $4: 51 ; 5: 47$.

- lucifuga $3: 36,47 ; 4: 50 ; 5: 45 ; 6-7: 16$.

— - var. gralla 6-7: 16 .

- maculata 4: 50:5: 43:6-7: 16

- microspora 5: 44 .

- mixtilis $4: 50 ; 5: 46 ; 6-7: 17,18$.

- napipes $1: 31,36,43 ; 2: 57 ; 3: 21,27,31,36,47,57 ; 4: 35$. $50 ; 5: 46 ; 6-7: 17$.

- oblectabilis 6-7: 17.

- obcura $3: 21,27,36,46,56 ; 4: 34 ; 5: 42$.

- - var. purpurea 5: 42 . 
- - var. rubens 5: 42.

- - var. rufa 5: 42.

- - var. violacens 6-7: 16 .

- ovatocystis $3: 31 ; 5: 47 ; 6-7: 17$.

— paludinella $4: 35 ; 5: 45,46$.

— patouillardii 1: 31,$43 ; 18$ (suppl.): 39 .

- perbrevis 6-7: 16 .

- perlata $3: 46 ; 5: 43 ; 6-7: 16$.

- petiginosa 5: 46;6-7: 17, 70 .

— phaeodisca 3: 65: 4: 50; 5: 44.

— phaeoleuca 6-7: 16 .

- pyriodora 3: 36; 5: 42.

- - var. incarnata 3: 65: 5: 42 .

- plumosa 3: 21, 36: 5: 46.

— posterula $3: 36,47,57,65 ; 4: 34,42,50 ; 5: 44$.

- praetervisa $3: 21,36,47,57 ; 4: 35,50 ; 5: 46 ; 6-7: 17$.

— - var.pusilla $3: 21,36 ; 5: 46$.

— proximella $3: 21,27,57 ; 5: 47 ; 6-7: 17,18$.

— pseudoasterospora $4: 35 ; 5: 46$.

- pudica 6-7: 16.

— pusio $3: 21,27,36,47,56 ; 4: 34,49 ; 5: 42$.

— putilla $3: 21,47,57 ; 4: 35,51,62 ; 5: 47 ; 6-7: 17$.

- relicina 5: 43; 6-7: 16 .

— repanda 6-7: 18 .

— rickenii 5: 46; 6-7: 16.

— rimosa 1: 30; 3: 21, 36; 5: 32, 43 .

- rimosoides 5: 43 .

- rubescens 6-7: 16.

— rufoalba 5: 46 .

- salicis 4: 35; 5: 46.

— sambucina $1: 31 ; 6-7: 16$.

— scabella 4: 50; 5: 46 .

- scabra 4: 34, 49; 5: 42 .

- serotina $5: 44$.

- sindonia 5: 42, 45 .

- squamata 5: 43 .

- subbrunnea 4: 50; 5: 44, 45; 6-7: 17.

- subrimosa 3: 21; 5: 46.

— tigrina 4: 42; 5: 44 .

— trechispora 4: 34; $5: 45$.

— trinii 6-7: 16.

- trivialis 5: 46

— umboninota $3: 21,31,36,47,57 ; 4: 35,50 ; 5: 46$.

- umbratica $3: 47 ; 4: 35 ; 5: 46 ; 6-7: 17$.

— umbrina $3: 57 ; 4: 35,50 ; 5: 46 ; 6-7: 17,70$.

- virgatula 5: 45 .

- viscidula $4: 49 ; 5: 42 ; 6-7: 16$.

— xanthomelas $3: 21,36,57 ; 4: 35,50 ; 5: 46 ; 6-7: 17 ; 17: 63$

Inoloma 2: 55, 57; 5: 51 .

- bolare 6-7: 70 .

- suillum 5: 54.

Inonotopsis 20: 14.

Inonotus 17: 34; 20: 2, 14.

- cuticularis 6-7: 42 .

- dryadeus 6-7: 42.

- dryophilus 6-7: 42

- hispidus 17: 34 .

— obliquus 8: 17; 10: 181 .

— radiatus $8: 17 ; 10: 181 ; 11: 31 ; 12: 48,49,63$.

— rheades 10: 181; 11: 31; 12; 47-51; 13: 45 .

Irpex 3: 42.

— fuscoviolaceus $4: 41 ; 5: 90 ; 8: 17$.

- lacteus 3: 53; 5: 90 .

- sinousus 3: 29, 42, 51; 5: 90.

— violaceus 4: 41; 5: 90 .

- woronowii 20: $2,5,7$.

Isaria 1: 83, 84.
- cretacea 1: 83,84

Isariopsis alborosella 17: 3, 15

Ischnoderma 5: 91 .

— benzoinum 3: 24, 42, 51; 4: 60; 5: 91; 6-7: 31.

- fuliginosum 4: 60 .

— resinosum 4: 60; 8: 17; 10: 182; 11: 31 .

- trogii 17: 38 .

Jaapia 14: 89.

Jafnea 17: 75 .

Junghuhnia collabens 20: 6 .

Karstenella 9: 20-22; 14: 109, 110, 124.

— vernalis 9: 20, 21; 14: 109.

Karstenellaceae 14: 109.

Krombholzia 2: 26.

- carpini 6-7: 70 .

- rufescens 2: 27.

- rugosa 6-7: 59 .

- scabra 2: 26.

- - var. coloratipes 2: 27.

- - var. nivea 2: 26.

- - var, roseofracta $2: 27$

- tesselata 6-7: 58.

Krombholziella 2: 26.

Kuehneola andicola 17: 6.

Kuehneromyces mutabilis $2: 58,61 ; 3: 22,28,31,40,49,59$; 4: $37,44,55 ; 5: 48,64,66 ; 8: 47,48,59 ; 9: 40-42,45 ; 11$ : 26,$31 ; 12: 47-50 ; 13: 45,46 ; 14: 50 ; 18$ (suppl.): $41,45,59$, $75,108,115,117$.

Laccaria 2: 54: $3: 14 ; 10: 8$.

— amethystina $2: 58 ; 3: 30 ; 4: 30,46 ; 5: 15 ; 6-7: 44,69 ; 8: 10$.

- echinospora $3: 20 ; 4: 30,46 ; 5: 15$.

- laccata 2: $58: 3: 7,12,14,20,26,30,33,44,54 ; 4: 30 ; 5$ : $15,103,116 ; 6-7: 37,44,68,111 ; 14: 50,51 ; 18$ (suppl.): 116,117

- - f. amethystina 3: 63 .

- - var. fuscata $5: 15$.

- - var proxima 3: 20

— - var. rosella $3: 20 ; 4: 46 ; 5: 15$.

— var. rufocarnea $3: 20 ; 5: 15$.

- nana $4: 46 ; 5: 15$.

— proxima $3: 54 ; 4: 30,46 ; 5: 15 ; 6-7: 8,37$.

- pumila $4: 46 ; 5: 15 ; 6-7: 8$.

- tortilis $3: 20 ; 4: 46 ; 5: 15$.

- trullisata 6-7: 111 .

- - ssp. (f.) maritima 6-7: 111.

- $f$. rugulospora 6-7: 111 .

Lachnea 5: 105; 6-7: 34.

— gemella 4: 59 .

- gregaria 4: 59; 5: 97

- hemisphaerica $3: 52 ; 5: 97 ; 6-7: 34$.

- melaloma 5: 105.

- miniata 5: 97.

— scutellata 3: 24, 30, 52, 62; 4: 41, 59; 5: 97.

- stercorea var. gemella 4: 41; 5: 97.

Lachnella confusa 2: 39,41 .

- pini 2: 39 .

Lachnellula 11: 42, 43.

- chrysophthalma 2: 40 .

- hahniana 11: 41 .

- occidentalis 11: 41-44.

— willkommii 11: 41-44.

Lachnobolus congestus 14: 78; 19: 10, 12, 14

Lacrymaria 1: 32,43 .

Lactarius 1: 17, 21, 24, 26, 32, 36, 39, 44, 46; 2: 9-11, 14, 15 , $21,23,48 ; 3: 6,8,9,14,16 ; 5: 6 ; 6-7: 36,65 ; 8: 47,48,56,59$; 10: 169,$171 ; 15: 21 ; 18$ (suppl.): 46-49, 51, 82, 108-111, $116,117$. 
- acerrimus 4: 58 .

— acris $2: 9-12 ; 6-7: 64 ; 17: 59,65$

— aspideus 2: 9-11.

— aurantiacus $1: 33,44 ; 2: 12,17,18 ; 3: 29 ; 5: 80 ; 6-7: 28,65$, 66.

- azonites 2: 10-12, 14, 21; 4: 40; 5: 79; 6-7: 56, 59, 61, 64, $69,71,73 ; 8: 12 ; 18: 3$.

- - f. albipes 5: 79 .

— badiosanguineus 6-7: 28 .

- blennius 2: 10; 6-7: 70; 17:65.

- bresadolianus 17: 65 .

- brevipes 15: 21 .

- brevis 15: 21

- camphoratus 1: 33,$44 ; 2: 10,12,18,22,58 ; 3: 7,50,61 ; 4$ : 58,$59 ; 5: 18,80,87$.

— chyrosorrh(o)eus 2: 10; 6-7:70; 15:21.

- cimicarius 1: 33, 44; $2: 13,21 ; 6-7: 62$.

- circellatus 2: 10, 13, 15 .

- colorascens 15: 21 .

- confusus $2: 13,17: 4: 40 ; 5: 79$.

— controversus $1: 33,44 ; 2: 10,13,58 ; 3: 23,28,41 ; 5: 78$; 6-7: 28,69 .

— cremor 2: 13; 3: 23, 61; 4: 40, 58; 5: 80; 6-7: 28.

- - var. laccatus 6-7: 28.

- ssp. pauper 2: 13.

- croceus 15: 21.

- cupularis $2: 18 ; 4: 58 ; 5: 80$.

— cyathula $2: 13,14,18,19,25 ; 3: 41 ; 4: 40,58 ; 5: 79,80$; 6-7: 28.

— decipiens $2: 10,13 ; 3: 61 ; 5: 81 ; 17: 65$.

- deliciosus 1: 18, 21, 33, 44, 85;2: 10, 13, 58:3:7, 9, 12, 14, $23,29,32,41,49,60 ; 4: 40,58,60 ; 5: 79 ; 6-7: 71 ; 8: 48$, 59; 9: 40, 41; 10: 171, 174; 16: 12; 18(suppl.): 5, 6, 41, 115, 117.

- dryadophilus 19: 42.

- duplicatus 15: 21.

- exsuccus 2: 13 .

- fascinans 2: 13 .

- flexuosus $1: 25,33,44: 2: 10,13,14,20,58 ; 3: 7,23,29$, $32,41,49,60 ; 4: 40,58 ; 5: 79 ; 6-7: 28.65$.

- - var. roseozonatus 6-7: 65

- fuliginosus 1: 33, 44; 2: 10-12, 14, 58; 3: 7, 41, 49; 5: 79; 6-7: 64, 65, 71.

_ - var. albipes $2: 12,14 ; 4: 40 ; 6-7: 64$.

- - ssp. azonites 2: 12 .

- - ssp. picinus $2: 14$.

- fuscus 2: 17: 4: 40 .

- geminus 2: 14, 16 .

- glaucescens 2: 10, 14, 19; 5: 79; 6-7: 59, 63, 64, 73; 8: 12 .

- glutinopallens 5: 79 .

- glyciosmus 1: 33, 44; 2: 10, 13, 14, 17, 58; 3: 7, 23, 29, 32, $41,49,60 ; 4: 40,58: 5: 79: 6-7: 70$.

- griseus 3: 60; 5: 79 .

- helvus 1: $25,33,44 ; 2: 10,14,15,21,58 ; 3: 7,23,29,32$, $41,49,50,61 ; 4: 40,58,59 ; 5: 18,80,87 ; 10: 18,64 ; 14:$ 49,$52 ; 18$ (suppl.): 41 .

- hepaticus $2: 10,11,14,15,58 ; 6-7: 28 ; 17: 65$.

- hibbardiae $2: 17 ; 4: 40,58 ; 5: 79$.

- hysginus $1: 88 ; 2: 10,13,15,58 ; 3: 23 ; 4: 58 ; 5: 79$.

— insulsus 2: 24; $4: 58 ; 6-7: 65$.

- isabellinus 15: 21 .

— lacunarum 4: 58; 5: 80; 15: 20, 21.

- lapponicus 15: 19-22, 28.

- lateritioroseus $2: 15,16: 5: 80$.

- lignyotus $1: 33,44 ; 2: 10,14-16,58 ; 3: 7.23,29 ; 5: 79$.

- lilacinus $1: 33,44 ; 2: 10,15,16,21,58 ; 3: 29,41,67: 4: 40$, 58; 5: 80; 6-7: 28 .
- - ssp. eulilacinus 2: 16

- luridus 2: 16, 17 .

- mammosus $2: 10,13,15,17,21 ; 3: 7 ; 4: 58 ; 5: 79 ; 6-7: 37$; 18(suppl.): 41 .

- mitissimus 1: 33, 44: 2: 10, 12, 17, 18, 22, 48, 49, 58; 3: 7, $29,41,49,60,67 ; 4: 40,58 ; 5: 80 ; 6-7: 28,44,60,63,65,71$. 72; 15: 19, 20, 21.

- $\mathrm{f}$. mitissimus 6-7: 28 .

- musteus 2: 10, 18, 22, 25 .

- necator $2: 10,11,18,22,58 ; 3: 23,29,32,41,49,60 ; 4: 40$, 58, 60; 5: 79; 6-7: 63; 9: 40-42, 44; 18(suppl.): 29-31, 41, $108,115,117$.

- obnubilus $2: 18,19,58 ; 4: 58 ; 5: 80$.

- obscuratus $2: 10,13,18,19,25,58 ; 3: 41,61 ; 4: 40,58 ; 5$ : $80 ; 6-7: 28$.

— pallidus 2: 10, 22; 17: 65 .

- pargamenus 2: 9, 19.

— picinus 2: 58: 3: 49, 60; 4: 58: 5: 79; 6-7: 28 .

— - var. speciosus 3: 49; 5: 79 .

- piperatus $1: 25,33,44 ; 2: 10,14,19,20,58 ; 3: 7,23,32$ $41 ; 5: 78,79 ; 6-7: 28,44,60-64,66,70,73 ; 8: 12 ; 17: 66$.

- - var. pargamenus 5: 79; 6-7: 28 .

- porninsis 4: 58 .

- pterosporus 6-7: 65; 17: 65 .

- pubescens $1: 33,44 ; 2: 10,19,22,58 ; 3: 60 ; 4: 40 ; 5: 79$; 6-7: 28,$70 ; 18$ (suppl.): 41 .

- pyrogalus $2: 10,13,19,58 ; 3: 7,29,67 ; 5: 80 ; 6-7: 42,61$, $64,66,70,73$.

- - ssp. communis 2: 19.

- - ssp. flexuosus $2: 13$

— quietus $1: 33,44 ; 2: 9,10,19,58 ; 5: 79 ; 6-7: 44,60-63,66$, $69,70,72,73 ; 8: 12 ; 18: 3 ; 18$ (suppl.): 41

- repraesentaneus $1: 33,44 ; 2: 10,11,19-21,58 ; 3: 7,23,29$, $41,49,60 ; 4: 40,60 ; 5: 79 ; 6-7: 68 ; 9: 40,41$.

— resimus $1: 33,44: 2: 10,20,58 ; 3: 29 ; 5: 79 ; 15: 20$.

- roseozonatus $2: 14,20 ; 6$-7: 65 .

- rufus 1: $25,26,33,40,44,85,88 ; 2: 10,11,15,17,21,58$; 3: $7,13,23,29,32,41,50,61 ; 4: 40,58 ; 5: 80 ; 6-7: 37,70$; 8: 59; 9: 40-42; 14: 49, 51, 52; 18(suppl.): 29-31, 41, 43, $45,81,82,97-99,107-109,111,115,117$.

— ruginosus 17: 59, 65 .

- scrobiculatus 1: $25,33,44 ; 2: 10,11,20,21,58 ; 3: 23,29$, $32,49,60 ; 4: 60 ; 5: 79 ; 6-7: 68 ; 15: 20,27 ; 17: 65 ; 18 ; 29$.

- - var. violascens $2: 20$.

- serifluus $2: 10,13,21 ; 5: 80 ; 6-7: 44,60,62,64,69,72,73$.

— sphagneti $4: 58 ; 5: 80,81 ; 6-7: 28$.

- spinosulus 2: $21 ; 3: 60 ; 4: 58 ; 5: 80$.

— subdulcis $1: 33,44: 2: 10,12,18,21,22,58 ; 3: 7,23,29$. $32,41,50,61,67 ; 4: 40,58 ; 5: 78,80 ; 6-7: 70 ; 17: 65$.

- substriatus 15: 21 .

- subumbonatus $2: 10,21 ; 6-7: 62$

— tabidus 2: 22: $3: 61 ; 5: 80,81 ; 6-7: 28$.

- thejogalus 1: 33,$44 ; 2: 10,11,18,21,22,58 ; 3: 50,61 ; 5$ : 81; 14: 51; 15: 20, 21; 18(suppl.): 41, 45 .

- torminosus 1: $18,23,25,26,33,40,44,87,88: 2: 10,11$ $16,19,22,58 ; 3: 7,23,28,32,41,49,60 ; 4: 40,58 ; 5: 78$, $79 ; 6-7: 63,70 ; 8: 56,59 ; 16: 12 ; 18$ (suppl.): 29-31, 35-37, $41,43-45,107,108,115,117$.

- - ssp. cilicioides 2: 22

- - ssp. eutorminosus 2: 19

- - var. gracillimus 2: 19.

- - ssp. pubescens 2: 19, 22

- trivialis 1: $15,25,26,33,40,44,85,87,88 ; 2: 10,11,22$ $58 ; 3: 7,23,29,32,41,49,60 ; 4: 40,58 ; 5: 79 ; 6-7: 37,65$, $68 ; 8: 39,56,59 ; 14: 51 ; 16: 10,12,13 ; 18$ (suppl.): $29-31$, $35-37,41,43,81,82,107,108,114,115,117$.

— turpis $1: 25,26,33,40,44 ; 2: 18,22,58 ; 3: 7 ; 5: 79 ; 6-7: 71$. 
— utilis 2: 22 .

- uvidus $1: 25,33,44 ; 2: 10-12,16-17,23,58 ; 3: 7,8,23,32$, 41,$49 ; 4: 58 ; 5: 79 ; 6-7: 72$

_ - var. subzonarius $2: 16,23 ; 3: 23 ; 4: 40 ; 5: 79$.

- - var. violascens $2: 16,23$.

- vellereus $1: 25,33,44 ; 2: 10,20,23,58 ; 3: 7,41 ; 5: 78 ; 6-7$ : $28,61,63,66,70 ; 8: 12 ; 17: 66 ; 18$ (suppl.): 6,7 .

- - var. bertillonii 6-7: 62 .

- var. velutinus 17: 65 .

— vietus $1: 33,44 ; 2: 10,11,23,58 ; 3: 7,29,41,49,60,67 ; 4:$ 40,$58 ; 5: 79 ; 14: 47,51,52$.

- violaceus 6-7: 70

- violascens 2: 16, 17 .

- volemus $1: 33,44 ; 2: 10,23,58 ; 5: 80 ; 6-7: 27,61 ; 8: 12 ; 17$ : 66.

— zonarioides 4: 58; 5: 79; 17:65

— zonarius $2: 10,24 ; 4: 58 ; 6-7: 46,65,69 ; 17: 65$.

- xanthogalactus 15: 21 .

- xanthydrorheus $15: 21$.

Laetiporus 20: 2 .

- sulphureus 6-7: 42; 8: 9, 17; 10: 179, 181; 11: 30, 31; 12: $47-51,63 ; 13: 46$.

Lamproderma 14: 68, 79; 18: 20; $19: 7$.

— arcyrioides 14: 54, 68; 19: 12 .

- arcyrionema 14: 68, 78; 19: 12, 15.

- atrosporum 14: 68, 78; 19: 12 .

- carestiae 14: 68, 78; 19: 12 .

- columbinum 14: 68,$79 ; 19: 12,13,15$.

- echinosporum 18: 20.

- griseum 18: 20, 21.

- gulielmae 18: 20, 21 .

- maculatum 18: 20; 19: 1, 5, 12.

- physaroides 19: 12 .

- puncticulatum 18: 20, 21; 19: 12

- robustum 14: 69

- sauteri 19: 1, 5, 12

— scintillans 14: 68, 78; 19: $1,6,12$.

— violaceum 6-7: 110; 14: 54, 68; 19: 12.

- - var. carestiae 14: 68.

- - var. sauteri 14: 54, 69 .

Lamprospora 13: 52 .

Leccinum 2: 26, 58; 9: 40; 17: 62; 18(suppl.): 108.

- aurantiacum $2: 27,28,53 ; 3: 43,54,62 ; 4: 29,45 ; 5: 8 ; 6-7$ : $6: 8: 58 ; 17: 61 ; 18$ (suppl.): 6 .

— carpini 3: 43; 4: 45; 5: 8; 6-7:59; 17:61.

- crocipodium 17: 61 .

- duriusculum 2: 26,$27 ; 4: 45 ; 5: 8 ; 6-7: 59 ; 10: 174$; 18(suppl.): 6.

- griseum 6-7: 56, 58, 59, 73; 8: 21 .

- holopus 4: 29, 45: 5: 8; 6-7:6.

- insigne 17: 61 .

- leucophaeum 3: 43, 54, 62; 4: 29, 45.

- - var. coloratipes $3: 43,54,62$.

- nigrescens 6-7: 58.

- oxvdabile 2: 27.

- quercinum 17: 59, 61 .

- rufescens 3: 43, 54,62.

- rufum $3: 19,25,30,33,43 ; 5: 8$

— scabrum 2: 26-28, 53; 3: 19, 25, 30, 33, 43; 5: 8; 6-7: 6. 37 59,$70 ; 8: 21 ; 9: 40-44 ; 10: 174 ; 14: 49,50 ; 18$ (suppl.): 6.7 , $12,41,116,117$

- - var. carpini 6-7: 59.

- - var. niveum $2: 27 ; 4: 45: 5: 8 ; 6-7: 6$.

_ - var. roseofractum 2: 27.

- subleucophaeum 17: 61, 62 .

- testaceoscabrum 2: 27, 28, 53; 3: 43, 62; 4: 29, 45; 5: 8; 6-7: 6. 37: 9: $39,41,42$.

- versipelle 14: 50, 51: 18(suppl.): 43, 45, 108, 115, 117.
- vulpinum 14: 49, 51; 18(suppl.): 29-31, 108.

Lentinellus auricula $5: 22$.

— bisus 3: 26, 55; 4: 47; $5: 22$.

- castoreus 5: 22 .

- cochleatus 3: 52; 5: 22.

— omphalodes $3: 20 ; 5: 22 ; 12: 47-50$.

— tridentinus 17:59, 69 .

- ursinus 5: 22.

- vulpinus 5: 22 .

Lentinus 1: $35,45,62,68-71,5: 23 ; 6-7: 10 ; 10: 181 ; 12: 50$; 17: 65 .

- adhaerens 5: 23 .

- bisus 10: 181 .

- cyathiformis 5: 23

- degener 17: 59, 64 .

- edodes 16: 3; 18(suppl.): 75.

- gallicus 6-7: 10 .

- lepideus $1: 61,68-71 ; 2: 58 ; 3: 20,26,30,34,45 ; 4: 31,60$; 5: 16,$23 ; 6-7: 10 ; 11: 29,31 ; 12: 47-51 ; 13: 40-43,46 ; 17$ : 59,65 .

- tigrinus 17: 65 .

- vulpinus 10: 181 .

Lenzites 3: 42; 5: 104

- abietina 5: 91 .

- betulina $3: 24,29.32,42,51,61 ; 4: 41,59 ; 5: 91 ; 8: 17 ; 10$ : 181; 11: 31; 17: 37 .

- heteromorpha $3: 32,61 ; 5: 91$.

— quercina $3: 51 ; 4: 28 ; 5: 91$.

- reichardtii 17: 37 .

- sepiaria 1: 61, 68-70; 3: 24, 29, 32, 42, 51, 61; 4: 41, 59; 5: $91 ; 8: 16 ; 11: 29 ; 13: 43$.

- - var. dentifera 20: $1,2,5$.

- tricolor 3: 42, 68; 5: 91 .

- warnieri 17: $33,37,38$.

Leocarpus contextus 19: 12 .

— fragilis 6-7: 110; 14: 61, 79; 19: 9. 12.

- vernicosus 19: 12

Leotia 3: 43; 5 : 95 .

- bulliardii 9: 24 .

- dicksonii 9: 24.

- elegans 9:24.

- gelatinosa $3: 43 ; 5: 95 ; 14: 116$.

- laricina 9: 24.

- lubrica 3: 43; 5: 95; 6-7: 66 .

- ludwigii 9: 24.

— uliginosa 9: 24.

- - var. aurantiaca 9: 24.

Lepidoderma 19: 5.

- tigrinum 14: 64, 79: 19: 12.

Lepiota 1: $28,41: 2: 52,58,59: 3: 9 ; 4: 60 ; 14: 87$.

— acutesquamosa $3: 52 ; 5: 36: 6-7: 69$.

- adnatifolia 14: 122 .

— alba $3: 35,46,56 ; 4: 49 ; 5: 36 ; 6-7: 14$.

- amianthina var. longispora 19: 25 .

- badhamii 17: 64 .

- bresadolae 17: 64 .

- clypeolaria 1: 28, 41:2:59;3:21, 28, 35, 46, 56:4:33,49, 60; 5: 36; 10: 180 .

— - var. ochraceosulphurescens $3: 56: 5: 36$.

— cristata 1: 28,$41 ; 2: 59 ; 3: 21,28: 5: 36 ; 6-7: 70: 14: 116$.

- cygnea 17: 59,64.

- echinata 8: 22 .

- eyrei 4: 21

- felina $2: 59: 3: 46: 5: 36$

- haematosperma 8: 22 .

- $f$. gracilis 8: 22 .

- helveola 1: 24, 28, 39, 41

- holosericea 17: 64 . 
- illinita 5: 36.

— jubilaei 17: 59, 64 .

— laevigata $3: 46 ; 4: 49 ; 5: 36$.

- leucothites 17: 64 .

- littoralis 17: 64.

- meleagroides 17: 64 .

- metulispora 6-7: 14.

— naucina $3: 7,35,46 ; 5: 36 ; 17: 64$.

—oreadiformis 3: 46; 4: 49; 5: 36; 6-7: 14.

- parvannulata 17: 64 .

- pratensis 3: 46 .

- procera 8: 46; 9: 40; 18(suppl.): 26, 27, 34.

— rhacodes $3: 7,13 ; 8: 46,48,49,58,59$.

- rhodorhiza 17: 64 .

- rufovelutina 17: 64 .

- seminuda 6-7: 14; 8: 21-23; 14: 116.

- setulosa 17: 64,

- - var. rhodorhiza 17: 64.

Lepista $2: 64 ; 10: 9,10 ; 11: 35,38,39 ; 14: 82-91,114,121$, 129-132; 15: 13, 14, 23; 18: 49-51, 53.

- auruginosa 15: 13, 14; 18: 50 .

- agrestis 15: 13, 14; 18: 52, 54.

- albofragrans 18: $49,52,54$.

— alexandri 10: 53.

— amarescens 15: 13, 14; 18: 53.

- ameliae 14: 83.

- argentina 14: 83.

— caespitosa $3: 64 ; 5: 29 ; 10: 114 ; 11: 39$.

- densifolia 14: 83; 18: 49, 50.

— diatreta 15: 13, 14; 18: 51, 52; 19: 22, 50 .

— fasciculata 14: 129, 130; 15: 14; 18: 52 .

- fragrans 15: 13, 14; 18: 50, 52-54.

- gilva 10: 9,$16 ; 14: 84,86-89 ; 15: 14 ; 18: 51-53$.

- harmajae 15: 13,$14 ; 18: 52$.

- idahoensis 15: 13, 14; 18: 50 .

- interveniens 10: 114 .

- inversa 18: 51 .

— irina 14: 83, 86, 90, 129-132; 15: 17; 18: 52.

- subg. Laevispora 14: 129.

- subg. Lepista sect. Disciformes 18: 49.

- - sect. Gilva 18: 49.

- - sect. Nuda 18: 49.

- luscina 11: 38, 39; 14: 83; 18: 52.

- marginella 15: 13, 14; 18: 52 .

- metachroa 15: 13, 14; 18: 49, 52, 53.

- metachroides 15: 13, 14; 18: 50, 53.

- nebularis 14: 82, 85, 91, 129-132; 18: 52; 18(suppl.): 114, $115,117$.

— nuda $2: 59 ; 3: 27,52,64 ; 4: 46,48 ; 5: 15,29 ; 8: 46,48,49$, 59; 10: 180; 11: 25, 26; 14: 82, 83; 18: 52, 53; 18(suppl.): $35-37,41,115,117$.

- odora 15: 13, 15; 18: 50-54: 19: 50.

— panaeola $3: 64 ; 4: 48 ; 5: 29 ; 14: 83$.

- personata 2 : $59 ; 3$ : 52; 5: 29; 6-7: 46; 18: 52; 18(suppl.): $115,117$.

- phyllophila 15: 13, 15; 18: 52-54.

- polycephala 15: 13,$14 ; 18: 52$.

— subg. Pseudolyophyllum 18: 49.

- - sect. Fragrans 18: 49.

- - sect. Metachroa 18: 49.

— - sect. Phyllophila 18: 49

- regularis 15: 13, 15; 18: 52, 54.

- rickenii 14: 83 .

- robusta 15: 13, 16:18: 50, 52 .

- singeri 14: 86, 129-132; 18: 52 .

— sordida $2: 59 ; 3: 27,34,64 ; 4: 32 ; 5: 29: 14: 83 ; 18: 52$.

- subaequalis 18: 49.

- subalpina 14: 82, 85, y1, 129, 130; 18: 52.
- subconnexa 11: $35,38,39 ; 14: 83,85-87,91 ; 18: 49-52$.

- truncata 3: 27; 5: 29.

Leptoglossum glaucum 3: 50; 5: 81 .

Leptomitus lacteus 12: 55.

Leptonia 1: 30, 42; 2: 59; 5: 32, 33.

Leptopodia murina 19: 43.

Leptoporus adustus 3: 24, 51, 61; 4: 41, 43, 59; 5: 90.

- - var. carpinus 6-7: 31 .

- - var. crispus 5: 90.

- $\mathrm{f}$. resupinatus 5: 90.

— albidus 3: 24, 29, 32, 51, 61; 4: 41; 5: 89; 6-7: 31.

- $\mathrm{f}$. guttulatus 5: 89 .

- albellus 4: 41; 5: 89 .

- amorphus $3: 24,32,61 ; 4: 41,43 ; 5: 90$.

— f. resupinatus 5: 90; 6-7: 31 .

— - var. vitreus 4: 43; $5: 90$.

- caesius 4: 43, 59; 5: 89 .

- chioneus 5: 89 .

- crispus 5: 90.

- dichrous 3: 61; 5: 90, 6-7: 31 .

- erubescens 5: 89 .

— floriformis 4: 59; 5: 89; 6-7: 31 .

— fragilis $3: 29 ; 5: 89 ; 6-7: 31$.

— imberbis 5: 90 .

- lacteus 3: 24, 29; 4: 40, 59; 5: 89.

— mollis 3: 29; 4: 41; 5: 89.

- semipileatus 5: 89 .

- semisupinus 5: 89.

- sericeomollis 5: 90.

- stipticus 3: 24, 51; 5: 89.

- tyromyces f. ptychogaster 5: 90.

Leucoagaricus 2: 59; 17: 64 .

- excoriatus 2: 59; $3: 35,52 ; 4: 33$.

- naucinus 17: 64 .

- pudicus 5: 36 .

Leucocoprinus 2: 59; 17: 64 .

- cepaestipes 2: 59 .

Leucocortinarius bulbiger 3: 28, 48; 4: 54; 5: 63.

Leucopaxillus 3: 63; 5: 20, 21; 10: 115; 14: 113.

- albissimus 3: 63; 5: 20.

- amarus 5: 20.

- - var. vulpeculus 5: 20; 6-7: 10.

- giganteus 5: 20.

- macrocephalus 17: 68 .

- mirabilis 5: 21 .

- otagoënsis 14: 113, 115.

- paradoxus 5: 20.

Leucoporus agaricus 5: 89 .

- arcularius f. scabellus 4: 43, 59; 5: 89 .

- f. strigosus 5: 89.

- brumalis 3: 24, 29, 32, 42, 51, 61; 4: 40, 49; 5: 88, 89 .

- $\mathrm{f}$. gracilis 5: 89 .

— - var. lepideus 5: 89 .

- - var. murinus 5: 89 .

- $\mathrm{f}$. trachypus 5: 89 .

- $\mathrm{f}$. vernalis 5: 89 .

Leucoscypha 13: 54; 14: 125: 17: 73, 75.

- albocincta 17: 75 .

- leucotricha 17: 75.

— ovilla 17: 73-75.

- patavina 17: 75 .

- rhodoleuca 17: 73-75.

- rutilans 17: 75 .

Licea 14: 79; 17: 31 .

— flexuosa 14: 71; 19: 12.

- fragiformis 19: 12, 14.

— minima 14: 71; 17: 22; 19: 12.

— variabilis 14: 71; 19: 12 . 
Limacella delicata 5: 36 .

— glioderma 5: 36 .

— illinita $3: 35 ; 5: 36$.

Limacium 1: 32,$44 ; 2: 57,59 ; 3: 8,10,14,16 ; 18$ (suppl.): 34.

— eburneum 3: 7, 8, 12, 14; 18(suppl.): 25-27, 34 .

- erubescens 3: 7 .

- hypothejum 3: 7 .

- olivaeoalbum 3: 7 .

- pustulatum 3: 7 .

Lindbladia effusa 14: 69; 19: 12.

- tubulina 19: 12.

Lindtneria trachyspora 8: 18 .

Lophodermium arundinacum 17: 3, 13.

Lycogala epidendrum 6-7: $110 ; 14: 58,60,72,79 ; 17: 31 ; 19$ : $8,9,12$.

- flavofuscum 14: 72, 78; 19: 12.

- miniatum 19: 8, 12.

Lycoperdon 5: 94; 6-7: 67; 13: 7; 14: 51; 18(suppl.): 34, 116, 117.

- atropurpureum 17: 70 .

- candidum 5: 94.

- caudatum 4: 58; 5: 94.

- depressum 17: 70.

- echinatum $3: 41,50,61 ; 5: 94 ; 6-7: 67,69,73 ; 8: 12$.

- ericetorum 17: 70.

- gemmatum 3: 8, 23, 29, 41, 50, 61; 4: 40, 58: 5: 94; 8: 48 .

- hirtum 3: 23 .

- molle 4: $58 ; 5: 94$.

- - var. atropurpureum 17: 70.

— nigrescens 3: 8; 5: 94.

- papillatum 3: 61; 5: 94.

— pedicellatum 4: 58; $5: 94$.

— perlatum $5: 94 ; 8: 37,47,59 ; 17: 70$.

- pyriforme $3: 23,29,32,41,50,61 ; 4: 40,58 ; 5: 94 ; 10: 180$.

- pusillum 6-7: 32.

— umbrinum $3: 23,29,41,50,61 ; 4: 40,58 ; 5: 94 ; 17: 70$.

- - var. asterospermum 3: 29.

Lyophyllum 2: 53, 54, 62, 64; 4: 62; 5: 20, 101-103; 6-7: 7; 10: $7,8,20 ; 14: 87 ; 15: 16-18 ; 18: 50 ; 18$ (suppl.): 116; 19: $24,51$.

— aggregatum $2: 59 ; 3: 20,44 ; 5: 12$.

- - var. ovisporum 5: 12 .

- ambustum 4: 45.

— atratum 5: $11 ; 6-7: 7$.

- baeospermum 3: 62 .

- capniocephalum $3: 63 ; 5: 12 ; 6-7: 7$.

- carbonarium 4: 45; $5: 11$.

- cessans $3: 25,44 ; 5: 11$.

- clusile 4: 29.

— conglobatum 2: 59; $3: 20,33 ; 4: 45 ; 5: 12$

— connatum 2: 59; 3: 25, 52; 5: 12; 18(suppl.): $41,117$.

— decastes $2: 59 ; 3: 20,25,44 ; 5: 12 ; 6-7: 7 ; 14: 130$.

- - var. ovisporum 3: 44; 4: 45; 5: 12.

_- var. sphaerosporum 3: 44; $5: 12$.

- erosum 4: 29, 45; 5: 11 .

— infumatum $3: 33,63 ; 5: 12 ; 14: 51$.

- inolens 5: 11; 6-7: 7 .

- leucomyosotis 4: 29.

- loricatum 9: 40-43.

- mephiticum 5: 11;6-7: 7.

- miserum 3: 63; 5: 11; 6-7: 7 .

- murinum 5: 11 .

- ozes 3: 62; $5: 11$.

- palustre 4: 29,$45 ; 5: 11$.

- piperatum 19: 22, 24.

— plexipes 4: 29, 45; 5: 11; 6-7: 7 .

- $\mathrm{f}$. atrum $4: 45 ; 5: 11$.

— putidum $3: 25,63 ; 5: 11 ; 6-7: 7$.

- rancidum 3: 33; 5: 11; 6-7: 7 .
- schulmannii 19: 50, 51.

- sphaerosporum 5: 11, 100-104

- striaepileum 3: 26, 33; 5: 12.

- subnitens 15: 16-18.

- suburens 19: 24.

- tesquorum 4: 45; $5: 11$.

- tylicolor 4: 45; 5: 11; 6-7: 7 .

— ulmarium $2: 59 ; 3: 25 ; 5: 12 ; 6-7: 8 ; 10: 181$.

- - f. verticale 5: 12 .

Lysurus gardneri 6-7: 109.

Macbrideola 17: 31 .

Macrocystidia cucumis 3: 26, 64.

Macrodiplodia bulbosa 19: 42 .

Macrolepiota 2: 59.

— excoriata 5: 36; 6-7: 14 .

— gracilenta 3: 52; 5: 36; 6-7: 14 .

- mastoidea 6-7: 14 .

— procera $2: 58 ; 3: 21,27,35,46,56 ; 4: 33 ; 5: 36 ; 9: 40,41$; 17: 64; 18(suppl.): 6, 39, 114, 115, 117.

- puellaris $3: 35 ; 5: 36$.

— rhacodes $2: 58 ; 3: 21,27,35,46,56 ; 4: 33 ; 5: 36 ; 10: 180$; 18(suppl.): 115, 117.

- - var. puellaris $3: 21,27 ; 5: 36 ; 6-7: 51$.

— umbonata $3: 52 ; 5: 36 ; 6-7: 14$.

Macropodia bulbosa 3: 24; 5: 96; 6-7: 34 .

- macropus 3: 62, 68; 4: 59; 5: 96; 6-7: 34 .

Marasmiellus ramealis 8: 10 .

Marasmiopsis 1: 78.

- subannulata 1: 77,78, 81. 82 .

Marasmius 1: 20, 30, 42, 78; 2: 52, 54, 59, 60, 62; 3: 5, 6, 9 , $14 ; 4: 5,8,60 ; 5: 24,102,107,112,114 ; 6-7: 11 ; 19: 59$.

- amadelphus 5: 24; 6-7: 11 .

— - var. abiegnus 5: 24 .

— androsaceus $2: 59 ; 3: 7,14,20,26,30,34,45,55 ; 4: 31,47$, $60 ; 5: 25,103 ; 6-7: 37 ; 9: 40,41 ; 10: 178,180 ; 13: 45 ; 14$ : 49-52: 17: 67; 19: 59, 60 .

— bulliardii 5: 25; 6-7: 11; 12: 63; 13: 45 .

- calopus 17: 67.

— candidus $3: 30 ; 5: 24 ; 17: 67$.

- caulicinalis 4: 6 .

- cauticinalis $4: 5,6$.

- cohaerens 6-7: 11; 14: 116.

- confluens 6-7: 49,69, 70 .

- conigenus 2: 28-30; 17: 68 .

- - ssp. esculentus $2: 29,3: 45 ; 4: 47 ; 5: 24$.

— epihyllus $2: 59 ; 3: 7,34,64 ; 4: 31,42,47,60 ; 5: 25,103 ; 10$ : 180.

- esculentus 2: 28, 29; 17: 68 .

- eufoliatus 5: 25; 6-7: 11 .

- foetidus 6-7: 11 .

— fulvobulbillosus 3: 26; 4: 5, 6; 6-7: 71 .

- globularis 3: 45; 5: 24 .

- graminum 3: 20; 5: 24, 112 .

- languidus 17: 67.

- lupuletorum 6-7: 11

- myosurus 2: 30 .

- nybergii 3: 20,$45 ; 5: 25$.

— oreades $2: 59 ; 3: 20,26,30,34,45,55 ; 4: 31,47,60 ; 5: 24$; 10: 180,$182 ; 18$ (suppl.): $6,116,117$.

- perforans $3: 7,8 ; 4: 47,60 ; 5: 15,24,103 ; 6-7: 11 ; 9: 40 ; 13$ : 45.

- peronatus 6-7: 69, 70; 17:67.

— prasiosmus 5: 24; 6-7: 48, 69, 72, 73; 8: 10; 10: 180; 12: 63 .

- putillus 3: 26 .

— ramealis $2: 59 ; 3: 45 ; 5: 24,48 ; 6-7: 11,48$.

- recubans $3: 26 ; 5: 25 ; 6-7: 11$.

— rotula $2: 59 ; 3: 20 ; 5: 25 ; 6-7: 11,49 ; 10: 180 ; 17: 67$.

- saccharinus 6-7: 11 . 
- scorodonius $2: 59 ; 3: 20,26,34,45,55 ; 4: 31,47,60 ; 5: 24$; 10: 180; 18(suppl.): 6, 116.

- siccus 15: 22.

- splachnoides 17: 67.

- subannulatus 1: 77, 78 .

- tenacellus 2: 29, 30.

- - ssp. conigenus 2: 29.

- - ssp. esculentus 2: 29 .

- tenerrimus $3: 20,45 ; 5: 25$.

- torquensis 6-7: 11 .

- tremulae 4: 31, 5: 25 .

— urens 3: 33; 6-7: 49; 13: 45; 19: 59, 60.

- wettsteinii 3: $20,45,52,55 ; \mathbf{5}: 25$.

- wynnei 3: 45, 5: 24.

Margarita metallica 14: 78; 17: 22; 19: 10, 12 .

Marssonia secalis 13: 23.

Mastigosporium 11: 5, 17, 19, 20; 12: 32; 15: 59.

- album 11: 5-10, 12-18, 20.

- deschampsiae 11: 5, 7-9, 13, 15-18, 20.

- rubricosum 11: 5, 7-20.

- - var. agrostidis 11: 14 .

Maublancomyces 16: 6 .

Melampsora epitea 8: 6 .

— euphorbiae 6-7: 95.

- medusae 17: 6 .

Melampsorella caryophyllacearum 17: 6 .

Melanoleuca 2: 64; 3: 64; 4: 47; 5: 21; 6-7: 10; 10: 7, 35; 11: 38.

— adstringens 5: 21; 6-7: 10 .

— amica $3: 64 ; 4: 47 ; 5: 21$.

— arcuata $3: 26 ; 5: 21$.

— aromatica 15: 16 .

- brevipes 3: 63; 5: 21; 6-7: 10.

- brevispora 18: 29,30 .

- cognata $4: 31,47 ; 5: 21 ; 6-7: 10$.

— evenosa 4: 31,$47 ; 5: 21$.

- excissa 6-7: 10.

— graminicola $3: 63 ; 5: 21$.

- grammopodia 2: 63; 5: 21 .

— - var, subbrevipes $3: 63 ; 5: 21 ; 6-7: 10$.

- luteolosperma 5: 21 .

- melaleuca 2: 60; 3: 20, 34, 45, 55, 63; 4: 31, 47; 5: 21 .

- microcephala 6-7: 10.

— oreina $3: 63 ; 5: 21$.

- polioleuca 3: 20, 26; 5: 21.

- pubifolia 3: 20 .

- strictipes 3: 20, 26, 30, 34, 45, 55; 4: 31, 47; 5: 21.

— stridula $3: 63 ; 5: 21$.

- subbrevipes 6-7: 10.

— turrita 3: 64; 5: 21.

- verrucipes 4: 47; 5: 21; 6-7: 10; 10: 114, 115

- vulgaris 2: 60,$34 ; 5: 21$.

Melanophyllum 8: 23 .

- echinatum 8: 22.

- eyrei 8: 21, 22

Melanopus 5: 88 .

— elegans 3: 53,61; 4: 28, 59; 5: 89 .

- $\mathrm{f}$. squamigerus $4: 28 ; 5: 89$

- melanopus 3: 24, 29, 42, 61; 4: 59; 5: 88.

- $\mathrm{f}$. brumaliformis $5: 88$.

- nummularius 4: 40; 5: 89 .

— picipes 3: 29.61; 4: 59; 5: 89 .

- squamosus $3: 24: 5: 88$.

— varius $3: 29,61 ; 4: 40,59 ; 5: 89$.

— - var. picipes 3: 24 .

- $\mathrm{f}$. squamigerus 4: 28, 59.

Melanospora 12: 61

- destruens 12: 61 .
Melastiza 9: 5, 6 .

— chateri 9: 6, 7 .

Meripilus giganteus 17: 36, 38 .

Merulioporia taxicola 5: 92; 8: 17.

Merulius 1: 62, 69.

- corium 5: 86

- domesticus 1: 61, 68-71.

- lacrymans 3: 53 .

- molluscus var. aurantiacus 3: 51; 5: 86.

- papyrinus 5: 86 .

- serpens 5: 92

- tremellosus 3: 29, 51, 67; 5: 86; 8: 17.

Metatrichia vesparium 19: 11-13.

Microdiplodia 19: 31 .

— cenangiicola 19: 30,31

Micromphale 2: 60; 4: 62 .

- brassicolens 17: 59, 67 .

- foetidum 17: 67 .

- perforans 2: 59; 3: 20, 26, 30, 34, 45, 55; 4: 31, 42; 9: 40 , $41 ; 10: 180 ; 14: 49,50 ; 19: 59,60$.

Microglossum elegans 9: 24 .

Microsphaera alni 4: 20.

- alphitoides 4: 20.

- baeumleri 4: 20.

- berberidis 4: 20 .

- betulae 4: 20 .

- divaricata 4: 20.

- evonymi 4: 20.

- grossulariae 4: 20

- lonicerae 4: 20.

- quercina 5: 98.

- viburni 4: 20.

Micronegeria fagi 17: 7 .

Microsporon 1: 48, 50.

Microstoma 13: 52, 53; 14: 125 .

- protractum 13: 52, 53, 55.

Mindeniella spironosa 12: 55 .

Mitrula 3: 43; 5: 95; 9: 23, 24, 29, 30 .

— abietis 9: 30, 32 .

- aurantia 9: 24.

- cucullata 4: 41; 3: 29; 5: 96.

— elegans 9: 24,29 .

— gracilis 9: 23-25, 27-33; 10: 42.

— - var. flavipes 9: 27.

- laricina 9: 24.

- multiformis 9: 29.

- muscicola 9: 27, 29, 30, 32.

— norvegica 9: 24 .

— omphalostoma 9: 30.

_ paludosa $3: 53,62 ; 5: 95 ; 9: 23-30,32,33$.

— - var. castanea 9: 24, 25.

- - var. cylindrica 9: 24,25 .

_- var. pachypes 9: 24, 25.

— $\mathrm{f}$. pallens 9: 24

— - var. sphaerocephala 9: 24, 25.

— phalloides 3: 53, 62: 5: 95; 9: 24, 29.

- - var. aurantiaca 9: 24.

— - var. pachypes 9: 24.

— pusilla 9: 23, 29 .

- rehmii 9: 27, 29, 30, 32.

Monilia 1: 54 .

Monilinia 20: 28, 30 .

Monospora 18(suppl.): 74

Montagnea arenaria 17: 70.

Montagnites 17: 71.

Morchella 5: 95; 6-7: 34; 9: 39, 46; 14: 110, 123, 124; 16: 2; 18(suppl.): $5,58,59,114,15,117$.

— conica $3: 30 ; 4: 28 ; 5: 95 ; 18$ (suppl.): 6 . 
- costata 5: 95 .

— elata 3: 30; 5: 95; 6-7: 34 .

- esculenta 5: 95; 17: 59, 71; 18(suppl.): 60.

- rotunda 18(suppl.): 6 .

Mortierella ramanniana 12: 59.

- pusilla var. isabellina 12: 59 .

Mucidula radicata 3: 20, 55 .

Mucilago 19: 5.

— crustacea 19: 9, 12, 13 .

- spongiosa 6-7: 110: 14: 64, 79; 19: 12.

Mucor 1: 56, 58, 59; 10: 179; 12: 61, 62, 64; 18(suppl.): 74, 86.

- dispersus 12: 60 .

- oblongisporus 12: 59-64.

- ramannianus 12: 65 .

Mutinus 6-7: 108, 109.

- caninus 6-7: 69, 108, 109.

Myceliophthora 14: 133, 135.

Mycena 1: 30, 42; 2: 28, 60; 3: 5, 9, 14, 34; 4: 8, 44, 48; 5: 23, $25,27,28,102,107-110,113-118,120 ; 6-7: 49,77-79,82$; 9: $19 ; 10: 7 ; 11: 25 ; 14: 49-52 ; 17: 68 ; 19: 53$.

- abramsii 6-7: 78 .

- acicula 6-7: 78.

- adonis 6-7: 77, 78 .

— aetites $3: 27,45,55,64 ; 4: 47 ; 5: 28$.

— alba $4: 48 ; 5: 28 ; 6-7: 12,78$.

- alboatra 5: 27.

- alcalina $2: 60 ; 3: 27,31,34,45,55,64 ; 4: 32,47 ; 5: 28 ; 6-7$ : $49,78,79,84-86 ; 17: 67$.

_- var. chlorinella $3: 34,55,64 ; 4: 32,42,47 ; 5: 27$.

- algeriensis 6-7: 78, 79.

- amicta 6-7: 77, 79.

- $\beta$ leucopsis 6-7: 79 .

— ammoniaca $3: 27,34,45 ; 4: 47 ; 5: 28$.

— atroalboides 5: 108, 109, 112, 113, 117; 6-7: 78, 79.

— atrocyanea 5: 27; 6-7: 80 .

- aurantiomarginata $3: 7,26 ; 4: 47 ; 5: 26,109 ; 6-7: 77,80$; 19: 53.

— avenacea $4: 47 ; 5: 27$.

- capillaripes 6-7: $80,86$.

— capillaris $4: 47 ; 5: 26$.

— cinerella 3: 7; 4: 47; 5: 28, 108, 109; 6-7: 11, 77, 81 .

— cineroides 6-7: 80, 81; 14:51.

- citrinomarginata $3: 27,45,55 ; 4: 47 ; 5: 26,109 ; 6-7: 77$, 80,81 .

- clavicularis 5: 108-110, 112, 113, 117; 6-7: 77, 78, 81, 82.

— concolor $3: 34 ; 5: 28$.

- corticola 2: 60; 5: 26; 6-7: 11, 82.

— crispula $3: 34 ; 5: 28$.

- debilis 6-7: 83 .

— delectabilis 3: 20; 4: 47; 5: 29; 6-7: 12, 82 .

- delicatella 6-7: 82 .

— elegans 2: 60; 3: 26; 4: 47; 5: 26; 6-7: 80, 81 .

- elegantula 6-7: 85 .

- epiphloea 6-7: 12.

—epipterygia $2: 60 ; 3: 7,13,14,27,34,45,55,64 ; 4: 31,47$; 5: $28,108-110,113,116-118 ; 6-7: 77,82,84 ; 10: 180 ; 14:$ 50 .

- - var. badiceps 6-7: 82 .

_ - var. brunneola 6-7: 82 .

- - var. lignicola 6-7: 82 .

— epipterygioides 5: 28; 6-7: 11 .

- erubescens 6-7: $12,78,82$.

— fibula $2: 60 ; 3: 34,45,55 ; 4: 32,48 ; 5: 28 ; 6-7: 77,82 ; 17$ : 67.

— filopes 3: 31, 64; 4: 32, 42, 47; 5: 26, 27, 108, 111-115; 6-7: $80,83$.

- flavoalba $2: 60 ; 3: 27,34,45,55,64 ; 4: 32,47 ; 5: 28,108$, $109,111,113,114,117 ; 6-7: 12,77,83$.
- - var. floridula 6-7: 12.

- floridula 6-7: 12.

- galericulata $2: 49,50,60 ; 3: 7,20,27,30,34,45,55 ; 4: 31$, 32,$47 ; 5: 26,28,108,109,111,116,117 ; 6-7: 49,77-79$, 83; 11: $26 ; 14: 50$.

- galopus (-poda) 2: 60; 3: 7, 13, 34; 4: 31; 5: 28, 108-110, $112-118 ; 6-7: 77,83 ; 10: 180 ; 14: 50 ; 19: 59,60$.

- - var. leucogala 6-7: 83 .

_ - var. (f.) nigra $3: 45 ; 4: 31 ; 5: 28$.

— gracilis 6-7: 83 .

- griseoviridis 6-7: 83 .

— gypsea 3: 34, 55; 4: 48; 5: 28.

- haematopus (-poda) $2: 60 ; 3: 34,45,55 ; 4: 31,47 ; 5: 28$, 109; 6-7: 77, 83; 14: 50.

- - marginata 6-7: 12,83 .

— hiemalis $4: 48 ; 5: 28 ; 6-7: 12$.

— ignobilis 4: 32; $5: 29$.

— inclinata $3: 45 ; 5: 26,108,109,111,115-117 ; 6-7: 49,69$, $72,78,83 ; 8: 10 ; 11: 26$.

— integrella 17: 67 .

— iodiolens 3: 30; 4: 31; 5: 26; 6-7: 78, 83 .

- - var. tenella 5: 26 .

- iris 6-7: 79 .

- jacobi 4: 32, 47; $5: 28$.

— janthina 3: 55; 5: 27.

- lactea $2: 60 ; 3: 7,13 ; 4: 48 ; 5: 28 ; 6-7: 82,84$.

- - var. pitya $3: 27 ; 5: 28$.

- laevigata 3: 45, 55; 4: 32, 47; 5: 27, 108, 109, 116-118; 6-7: 77, 78, 84; 13: 45; 18(suppl.): 41 .

— leptocephala $3: 34,55 ; 4: 32,47 ; 5: 27,109 ; 6-7: 84$.

- lineata $3: 7,14,45,55 ; 5: 26$.

- longiseta 5: 108, 110; 6-7: 77, 78, 84 .

- luteoalcalina 5: 108-110, 113-116; 6-7: 77-79, 84; 10: 182.

- maculata 3: 26, 64; 5: 26, 108, 109, 111, 115-117; 6-7: 77, 78,$84 ; 11: 26$.

- majalis $2: 28$.

— megaspora 4: 31; 5: 26, 108-110, 112-114, 117; 6-7: 77, 84 .

— metata $2: 60 ; 3: 7,26 ; 5: 26,108 ; 6-7: 77,78,80,83$.

— mirata 5: 26; 6-7: 85 .

— niveipes 4: 47; $5: 28,109 ; 6-7: 12,78,79,85$.

_ olida 4: 48; $5: 28$.

— olivascens 3: 55, 64; 5: 26.

- oregonensis 6-7: 85 .

— parabolica $3: 26,64 ; 5: 26 ; 6-7: 11,85$.

- - f. langei 4: 47 .

- pearsoniana 6-7: 85 .

- pelliculosa 5: 28 .

- permixta 4: $31 ; 5: 26 ; 6-7: 11$.

- phaeophylla 4: 32; 5: 28 .

— picta 19: 52, 53 .

- pinetorum 6-7: 82 .

- pitya 5: 28 .

- plicosa 4: 47; 5: 27.

- plumbea 6-7: 86 .

- polyadelpha 5: 26 .

- polygramma $3: 20,27 ; 4: 47 ; 5: 26,27,108,111-116,118$; 6-7: 49, 85 .

- - f. candida 5: 27.

— f. pumila 4: 32; 5: 27.

- praecox 4: 44, 47; 5: 28.

- praelonga 6-7: 84 .

- pseudocorticola 5: 26; 6-7: 85 .

— pseudogalericulata 4: 32, 47: 5: 28; 6-7: 85 .

- pseudolactea 6-7: 82.

- pseudopicta 4: 30; 6-7: 12.

- pseudopura 4: 31,$47 ; 5: 27$.

- pterigena 6-7: 77, 85; 15: 27.

- pura $2: 60 ; 3: 7,13,14,20,27,34,45,55 ; 4: 31,47 ; 5: 27$, 
$108,109,116,119,120,121 ; 6-7: 12,70,77,85 ; 14: 50$

— - var. alba 6-7: 85.

— - var. lutea 4: 47; 5: 27.

- - var. rosea $5: 27 ; 6-7: 85$.

- purpureofusca $3: 55 ; 4: 31 ; 5: 27 ; 6-7: 85$.

- rigidula 6-7: 85 .

- rorida $2: 60 ; 6-7: 12,77,86 ; 14: 50$.

— rosella $2: 60 ; 3: 7,12,14,27,30,34,45,55 ; 4: 31,60 ; 5: 27$, 108-110, 113, 117; 6-7: 77, 86.

- rubromarginata $2: 60 ; 3: 45,55 ; 4: 31,47 ; 5: 27 ; 6-7: 12$, 80,$86 ; 10: 182$.

— rugosa $3: 34,45,55 ; 4: 31 ; 5: 26 ; 6-7: 82$.

— salicina 6-7: 86 .

— sanguinolenta $2: 60 ; 3: 7,12 ; 4: 31,47 ; 5: 28,108,109$, $112-115,117,118 ; 6-7: 77,86 ; 10: 180,182 ; 17: 68$.

— sepia 3: 20; 5: 26; 6-7: 79, 80.

- speirea 4: 32; $5: 28 ; 6-7: 12,86$

- stannea 6-7: 84 .

— strobilicola 2: 28,$29 ; 6-7: 77,86$.

- stylobates 5: 108, 109, 111, 113, 114, 117; 6-7: 77, 78; 17: 68.

- supina 5: 26.

- swartzii 2: 60; 4: 32, 48; 5: 26, 28; 6-7: 12, 77, 86.

- tenella 5: 26, 108-110, 112-114, 117 .

- tintinnabulum 5: 26; 6-7: 86 .

- uracea 5: 26 .

- urania 5: 108, 110, 113, 117; 6-7: 77, 86 .

- vernalis 2: 28, 29.

— viridimarginata 6-7: 84

- - var. lutea 6-7: 84 .

— viscosa 4: 31; 5: 28; 6-7: 11, 77, 83, 86; 10: 180.

— vitilis $3: 26,30,31,45,64 ; 4: 31,32,42,47 ; 5: 26,27,108$, $109,111,113,114 ; 6-7: 83,86$.

- - f. caespitosa 6-7: 11 .

— vitrea $3: 20 ; 5: 26 ; 6-7: 79$.

- - var. tenella 6-7: 84 .

— vulgaris $2: 60 ; 3: 7,13,27 ; 4: 47 ; 5: 28,108-110,111,113$, $114,116,117 ; 6-7: 12,77,78,82$.

— zephirus 3: 64; 5: 27.

Mycenella bryophila 3: 55; $5: 23$.

- lasiosperma 3: 55; 5: 23.

- margaritispora 4: 47; 5: 23.

- salicina 4: 47; 5: 23.

- trachyspora 3: 55; 5: 23.

Mycogone perniciosa 18(suppl.): 19, 85.

Mycosylva 14: 133, 135.

- clarkii 14: 133-136.

- reticulata 14: 133-136.

Mycoleptodon fimbriatum 5: 87 .

- ochraceum 6-7: 30.

Myxacium 2: 60, 39; 4: 54; 5: 58.

- betulinum 5: 58 .

- causticum 3: 66; 4: 54; 5: 59; 6-7: 22.

- collinitum 2: 55; 3: 22, 28, 39, 48, 58; 4: 45; 5: 58; 6-7: 22.

- - var. subolivascens 4: 54; $5: 58$.

- delibutum 2: 55; 3: 22, 28, 31, 39, 48, 58; $5: 58$.

— elatior 2: $55,58,66 ; 4: 54 ; 5: 58 ; 6-7: 22$.

- elatium 3: 48; 4: 36 .

— epipoleum 5: 58; 6-7: 22.

- illibatum 5: 58 .

- metrodii 6-7: 20 .

- mucifluoides 4: 36; 5: 58 .

- mucifluum $3: 22,28,48,58 ; 4: 36 ; 5: 58$.

- mucosum $3: 22,28,31,39,48,58 ; 4: 36,54 ; 5: 58$.

- - var. coeruliipes 5: 58 .

- nitidum 5: 58.

- pluviorum 5: 59; 6-7: 22.

— pluvium 3: 48,$58 ; 5: 58$.
— pseudosalor 4: 36; 5: 58; 6-7: 22.

— salor 4: 43; 5: 58 .

— stillatitium 3: 58; 4: 36, 54; 5: 58; 6-7: 22.

- triviale 5: $58 ; 6-7: 22$.

- vibratile $2: 55 ; 3: 28,39,48,58 ; 5: 58$.

Myxotrichum resinae 14: 135 .

Naematelia encephala 3: 29; 5: 81 .

Naematoloma 2: 57, 62; 4: 37; 5: 66, 67; $14: 114$.

— capnoides $2: 60 ; 3: 22,28,31,40,49,59 ; 4: 37,55 ; 5: 66$; 6-7: $37 ; 8: 37,47-49,51,58-60 ; 18$ (suppl.): 29-31, 59, 108, $114,115,117$.

— dispersum 4: 55; 5: 66; 6-7: 25.

— elaeodes $4: 55 ; 5: 66 ; 6-7: 25$.

- elongatum 2: 60; $4: 37,55 ; 5: 66 ; 6-7: 25$.

- epixanthum 4: $55 ; 5: 66 ; 6-7: 25$.

— ericaeum 4: 38; 5: 66, 67; 6-7: 25 .

- fasciculare $2: 60 ; 3: 22,28,31,40,49,59 ; 4: 37,55 ; 5: 66$; 8: $46 ; 18$ (suppl.): 39,41 .

- f. pumilum 5: 66 .

- - var. pusillum 6-7: 51 .

- inopum 6-7: 25.

— polytrichi 2: 60; 4: 55; 5: 66 .

— radicosum 4: 55; 5: 66; 6-7: 25 .

— sublateritium 2: $60 ; 3: 22,28,40,49,59,66 ; 4: 37,55 ; 5$ : 61,$66 ; 6-7: 51 ; 18$ (suppl.): 41 .

- - var. pomposum 5: 66 .

- subpapillatum 5: 72 .

- udum 4: 37; 5: 66; 6-7: 25.

- - f. sphagnicola $4: 37 ; 5: 66$.

Nannfeldtiella 9: 5.

- aggregata 9: 5 .

Napicladium arundinaceum 15: 42.

Naucoria 1: 31; 2: 60; 6-7: 18.

- centuncula var. luxurians 6-7: 18 .

- cerodes 3: 37; 5: 48; 6-7: 18, 19.

- confragosa 18: 55 .

- crobula $4: 38$.

— erinacea 4: 35 .

- fulgens 5: 48 .

- laevigata 6-7: 18

- myosotis 3: 31 .

- reducta 4: 44; 5: 48.

- segestria $3: 37 ; 5: 48$.

- riparia 17: 64.

Nectria cinnabarina 4: 43, 44, 60; 5: 98.

Nematospora gossypii 12: 65 .

Neogyromitra 9: 9-11; 13: 48-56;15: 29, 34; 16: 6, 7.

— caroliniana 9: 10; 13: 52; 15: 33; 16: 7 .

— gigas 9: 10; 13: 52; 16: 7 .

Neottiella 17: 75 .

—ovilla 17: 73 .

- patavina 17: 75 .

- rutilans 17: 75.

Neottiopezis macrospora 17: 75.

Neurospora crassa 14: 5, 7 .

Nidularia confluens 3: 29; 4: 40; 5: 93.

- farcta 3: 29; 5: 93.

Nolanea 1: $30,42,2: 60 ; 4: 48 ; 5: 32 ; 17: 63$.

- sericea 17: 59.

Nyctalis 2: 60 .

- asterophora 3: 20; 5: 11 .

Ochromitra 14: 110.

Ochrosporus pomaceus 17: 77.

- lithuanicus 20: 1, 8, 10, 12.

Octospora 13: 52; 14: 109.

- aggregata 9: 5 .

Odontia 5: 85.

- corrugata 5: 86 . 
- papillosa 5: 86 .

Oidium 4: 14, 21, 25; 17: 13.

- campanulae 4: 21 .

- chrysanthemi 4: 21.

- cyparissiae 6-7: 100.

- hortensiae 4: 21.

- hyssopi 4: 21 .

- lini 4: 21 .

- ruborum 4: 21 .

Oligonema aeneum 14: 78; 19: 10, 12, 14

Omphalia 1: 30, 42; 2: 60; 5: 14, 25, 103, 104; 10: 7 .

- campanella 3: 7; 4: 5, 6 .

- - var. badipus 4: 6 .

- candicans 10: 73 .

— crispula 3: 34; 5: 28.

- fibula $3: 34 ; 5: 28$.

- gracillima 3: 20; 5: 29.

- giovanellae 4: 32 .

— grisea 4: 47; 5: 28

- leucophylla 3: 63 .

- maura 5: 100-104.

- philonotis 4: 31 .

— picta 19: 52

_ - var. concolor 3: 34; 5: 28.

- reclinis 3: $25,44$.

- scyphoides 3: 27, 33, 34.

- setipes 4: 32; $5: 28$.

- speirea var. tenuistipes 6-7: 12.

- stellata 5: 29.

— striaepilea 3: 26, 44; 5: 15, 25.

- striatula 5: 14 .

Omphaliella muralis $3: 63 ; 4: 62$.

— rustica $3: 63 ; 4: 62$.

Omphalina $2: 60 ; 3: 34,44 ; 4: 5,8,62 ; 5: 17,18 ; 10: 9-12$, 32,$41 ; 11: 35 ; 15: 23 ; 17: 68 ; 19: 51$.

— abiegna $3: 20 ; 5: 17$.

- atropuncta 5: 18 .

- bibula 3: 20; 5: 17 .

- bresadolae 5: 18; 6-7: 9 .

- chrysophylla 2: 60; $3: 52,54 ; 5: 17$.

— epichysium 2: 60; 5: 18 .

- ericetorum 6-7: 37; 14: 50, 52.

— fibula 2: 60 .

— grisella $3: 55 ; 5: 17 ; 17: 68$.

— griseopallida $4: 30 ; 5: 17,18 ; 6-7: 9$.

- hydrogramma 14: 114

— invita $3: 26 ; 5: 18 ; 6-7: 9$.

- jalapensis 11: 40; 14: 115 .

- maura 2: 60 .

- obscurata $4: 30 ; 5: 17,18 ; 6-7: 9,12$

— oniscus $3: 45 ; 4: 30,31,46 ; 5: 18$.

— philonotis $3: 34,45 ; 4: 46 ; 5: 18,25 ; 6-7: 9$.

- postii 6-7: 9 .

- pseudopicta 4: 30; 5: 18 .

— pyxidata $2: 60 ; 5: 18 ; 6-7: 9,15$.

- reclinis $4: 30 ; 5: 18 ; 6-7: 9$.

- rosella $5: 18$

- rustica 4: 30; 5: 17, 18, 33, 81; 6-7: 9.

— scyphiformis $3: 44,55 ; 4: 46 ; 5: 17$.

- scyphoides 5: 17; 6-7: 9.

— sphagnicola 2: $60 ; 3: 55 ; 5: 18 ; 14: 52$.

- subfumosa 19: 50, 51 .

- swartzii 2: 60 .

- umbellifera 2: 60; 3: 20, 34, 54; 4: 30, 46; 5: 17.

— - var. albida $3: 20,54 ; 4: 30,46 ; 5: 17$.

_ - var. citrina $3: 20 ; 5: 17$.

- velutina 17: 68 .

Onnia 20: 2
Oospora 9: 46; 15: 10 .

Ophiobolus sativus 12: 26.

Ophiostoma multiannulata 12: 57.

Opisteria arctica 2: 28.

Otidea $3: 43,52,62 ; 5: 96 ; 13: 51 ; 14: 102,103,105-107$, $110,111,123-125,138-142 ; 15: 28-32 ; 17: 46,49,54$.

- abietina 15: 32; 17: 46 .

— alutacea 14: 138; 15: 31 .

- - var. microspora 15: 32

- apophysata 14: 139.

- auricula 14: 102; 17: 46 .

- bufonia 3: 24; 5: 96; 14: 138 .

— caligata 10: 41; 14: 138; 15: 32.

- cantharella 14: 105-107.

- - var. minor 14: 107

- cochleata 5: 96; 6-7: 70 .

- concinna f. integra 14: 107 .

— felina 3: 62; 5: 96 .

- formicarum 15: 29, 31, 32; 19: 52 .

- indivisa 13: 55; 14: 103, 119, 138-141; 15: 21, 28, 32; 17: 46 .

- kauffmanii 14: 139

- leporina $3: 30,52 ; 5: 96 ; 13: 55 ; 14: 51,138 ; 15: 30 ; 19$ : 52.

- microspora 15: 29, 32.

- myosotis 15: 29, 32.

— nannfeldtii 15: 29,31 .

- onotica 3: 52; 5: 96; 6-7: 34, 70; 13: 55; 14: 105, 138, $140,141$.

- papillata 15: 29,31

- phlebophora 13: 55; 14: 105-107, 139.

- platyspora 14: 138, 142 .

- propinquata 15: $21,28,29,32 ; 17: 46 ; 18: 29$.

- rainierensis 14: 139.

- silvicola 14: 102, 103.

- tuomikoskii 15: 29-31.

Oudemansiella 6-7: 48 .

- platyphylla 4: 47; 5: 23; 6-7: 11,$13 ; 10: 7 ; 14: 50$; 18(suppl.): 41.

- longipes 6-7: 46 .

— radicata 5: 23; 6-7: 47, 56, 69, 73; 8: 10; 10: 7; 17:68.

Ovularia 15: 39.

— pulchella 15: 38 .

- pusilla 15: $38-41$.

Oxyporus corticola 18: 47 .

- obducens 8: 17.

- populinus 8: 17 .

Panaeolina 2: 61, 62; 5: 70

- foenisecii $2: 61 ; 3: 22,40,49,60 ; 4: 38,56 ; 5: 70$.

Panaeolus 1: $24,32,39,43 ; 4: 38,56 ; 5: 68,69 ; 6-7: 25$, 26; 10: $180 ; 17: 62$

- acuminatus $3: 22,40,59 ; 4: 38,56 ; 5: 68,69 ; 6-7: 25$, 26.

- campanulatus $1: 23,32,43 ; 2: 61 ; 3: 22,28,40,49,59$; 4: 38,$56 ; 5: 68,69 ; 6-7: 25,26$.

— fimicola $3: 22,40,49 ; 4: 38,56 ; 5: 68$.

- - var. ater 3: 40,60, 67; 5: 69 .

- foenisecii 1: 32,$43 ; 2: 61$.

- olivaceus 17: 62 .

- papilionaceus $1: 23,32,43 ; 2: 61 ; 3: 22,59 ; 5: 68 ; 6-7$ : 25; 17: 62.

- phalaenarum 6-7: 26; 17: 62 .

- retirugis 1: 32,$43 ; 3: 22,40 ; 4: 38,56 ; 5: 68 ; 6-7: 25$.

- semilanceatus 2: 61 .

- separatus 2: 61 .

- solidipes 17: 62 .

- sphinctrinus 3: 40, 49, 59; 4: 38, 56; 5: 68, 69 .

— subbalteatus 3: 40, 49, 59; 4: 38; 5: 68 . 
- teutonicus 17: 59, 61, 62 .

Panellus 2: 61, 62 .

- mitis $2: 61 ; 3: 26,34,64 ; 4: 31,47 ; 5: 23 ; 11: 31 ; 12: 48$, 49.

- serotinus 11: $31 ; 12: 47-49 ; 19$ (suppl.): 41 .

- stypticus (stipticus) $2: 61 ; 5: 22 ; 6-7: 10,48 ; 10: 181$; 11: $31 ; 12: 48,49 ; 17: 68$.

— violaceofulvus $5: 23$.

— violaceofuscus 4: 42 .

Panus 1: 30, 42; $2: 61 ; 5: 23$.

- carneotomentosus 5: 23.

- conchatus $2: 61 ; 3: 20,26,30,34,45,55 ; 4: 31,47 ; 5$ : $23 ; 10: 181,182 ; 11: 31 ; 12: 48,49,63$.

— rudis 6-7: 10 .

- stypticus (stipticus) 1: 30, 42;2:61.

- torulosus 2: 61; 5: 23.

Paradiacheopsis 17: 26, 27, 31 .

- fimbriata 17: 19, 26, 27, 29-31, 87, 88; 18: 60; 19: 12 .

- solitaria 17: 19, 26, 27; 19: 12.

Paradiscina 15: 33, 34 .

— intermedia 13: 51, 53, 54; 15: 30, 33.

- melaleuca 15: 34.

Passsalora graminis 13: 13 .

Patella ovilla 17: 73, 74 .

Paxillus 1: 31, 76; 2: 61; 4: 46; 5: 13, 29; 6-7: 10; 14: 90, 91.

— atrotomentosus $2: 61 ; 3: 7,19,25,30,33,43,54 ; 4: 29$; 5: 8; 9: 40, 41; 18(suppl.): 41.

— extenuatus $3: 20,33 ; 5: 12 ; 14: 90$.

- filamentosus 1: 76, 77; 3: 54, 62; 4: 29, 45; $5: 8$.

- inornatus $3: 33 ; 5: 12$.

- involutus 1: 21, 76, 77; 2: 49, 61; 3: 7, 9, 13, 14, 19, 25, $30,33,43,54 ; 4: 29,45 ; 5: 8 ; 6-7: 70 ; 9: 40-42 ; 10: 74,172$; 14: $9 ; 18$ (suppl.): $6,39,41$.

— lepista 3: 27; 5: 29; 14: 90, 91; 18: 49.

- leptopus 1: 76; 3: 54; $5: 8$.

— panuoides $2: 61 ; 3: 25 ; 5: 8$.

- sordarius 14: 90.

Paxillopsis 1: $31 ; 2: 61$.

Paxina acetabulum 19: 37.

— platypodia 19: 44.

- recurva (-um) 18: 57; 19:43, 46, 48, 49.

- sulcata 19: 35.

Pelloporus corrugis 17: 38 .

Peltigera canina 19: 20.

- leucophlebia 19: 42.

Penicillium 1: 56, 58-60; 10: 179; 18(suppl.): 51.

— digitatum 12: 55, 57.

- griseofulvum 11: 25.

Peniophora 4: 40, 58; 5: 84; 6-7: 29.

— alnea 5: 85 .

— aurantiaca 5: 85 .

— gigantea 5: 85

- guttulifera 6-7: 29.

- incarnata 4: 40, 44, 58; 5: 85

- laevis 6-7: 29.

- lilacea 5: 85 .

- macrospora 6-7: 29.

- mutata 4: 43; $5: 85 ; 6-7: 29$.

- polygonia 6-7: 29.

— proxima 5: 85 .

- roumeguerii 5: 85 .

— rudis 5: 85 .

- setigera 5: 85 .

- subascondita 5: 85

- subcremea 4: 61; 5: 84.

- sublaevis 6-7: 29.

— tomentella f. depauperata 4: 58; 5: 85 .
— velutina 4: 43; 5: 85 .

- versicolor 5: 85 .

Perichaena 14: 79.

- chrysosperma 17: 19, 22; 19: 12 .

- corticalis 14: 78-80; 19: 12, 15.

- phaeosperma 19: 11, 12, 14.

- populina 19: 12.

- rostafinskii 19: 12, 15.

Peronospora 8: $5 ; 17: 4$.

- aestivalis 17: 3 .

- alsinearum 8: 6.

- alta 8: 6.

- chenopodii 17: 3.

- dentariae 17: 3.

- grisea 17: 3

- lapponica 17: 3, 4 .

- mayorii 8: 6 .

- minor 17: 3, 4 .

- parasitica 8: 6 .

- polygoni 8: 6 .

— ranunculi $8: 6 ; 17: 4$.

- ranunculi-peduncularis $17: 1,4$.

- - var. ranunculi-minutiflori 17: 1, 4.

- sepium 8: 6 .

- trifolii-repentis $17: 3,4$.

Peziza 1: 12; 9: 11; 13: 52, 54, 56; 14: 117, 123, 125; 15: 33; 16: $7 ; 17 ; 50,74 ; 18$ (suppl.): 54.

— abietina 15: 32 .

- acetabulum 6-7: 72, 108, 109.

- albocincta 17: 75.

- amphora 17: 41.

- badia 13: 55; 14: 126.

- bulbosa 17: 40, 42 .

- calyx 17: 41 .

- calyx* minor 17: 41 .

- cantharella 14: 107.

- debeauxii 17: 41, 42.

- gerardii 19: 38 .

- laetissima 9: 6 .

- nivea 17: 75.

- ochracea 14: 119.

— ovilla 17: 73, 75 .

- percevalii 17: 41

- perlata 9: 11; 13: 56.

- phlebophora 14: 105, 107.

- propinquata 15: 32.

- proteana 13: 51; 14: 103.

- protracta 6-7: 105.

- solitaria 17: 40, 42.

- spurcata 14: 116, 117, 119, 120.

- verruculosa 18: 57; 19: 33, 43, 44.

- warnei 13: 56.

Phacidium infestans 2: 39; 12: 64; 14: 43, 97.

Phaeocollybia 6-7: 14, 18.

— christinae $3: 57 ; 5: 50 ; 6-7: 18$.

— cidaris 5: 50 .

- - var. minor 6-7: 18 .

— festiva 4: 35; 5: 50; 6-7: 18 .

- jennyae $3: 22,57 ; 4: 51 ; 5: 50$.

- lugubris 6-7: 18.

Phaeolepiota aurea 5: 39; 10: 180.

Phaeolus 17: 34, 35; 20: 2, 14.

— nidulans 3: 24; 5: 90.

- rutilans $3: 24,51,61 ; 4: 44 ; 5: 90$.

- schweinitzii $3: 51,53 ; 4: 41 ; 5: 90 ; 6-7: 31 ; 8: 18 ; 10: 178$, 182; 17: $34,35$.

Phaeomarasmius 17: 64; 18: 55 .

- aridus $4: 35 ; 5: 48$. 
- carpophiloides 4: 51; 5: 48 .

— confragosus $5: 48,49 ; 14: 50 ; 18: 55,56$.

- erinaceus 5: 48 .

- limulatus 4: 51; 5: 48.

Phallus hadriani 6-7: 109.

— impudicus 3: 23; 5: 94; 6-7: 36, 108, 109.

Phellinus 12: 50; 17: 35, 77, 83; 20: 2 .

- conchatus 8: $18 ; 10: 181 ; 12: 48,49$.

- ferruginosus $8: 18 ; 10: 181$.

- friesianus $4: 43 ; 5: 92$

— igniarius $3: 24,30,32,42,51,61 ; 4: 41,43,44,59 ; 5: 91$, $92 ; 8: 18,23 ; 10: 181 ; 11: 31 ; 12: 48-50,63 ; 13: 45 ; 17: 35$, $77,79,80,83$.

- - var. igniarius 17: 35,82 .

- - var. resupinatus 4: 43; 5: 92.

- - f. sorbi 5: 91 .

— - var.(f.) tremulae 4: 44; 5: 91.

— isabellinus 8: 18 .

- laevigatus 8: 18; 17: 83 .

— nigricans 4: 59; $5: 92$.

— nigrolimitatus $4: 59 ; 5: 92$.

— pini 8: 18; 10: 182; 11: 29,$31 ; 12: 47-51 ; 13 ; 45$.

- - var. abietis 8: 18

— pomaceus 8: 18; 10: 181; 17: 35, 77-84.

- - var. oleae 17: 80 .

- - var. prunastri 17: 80 .

- populicola 17: 84 .

- punctatus $4: 43 ; 5: 92 ; 8: 18 ; 10: 181 ; 12: 48,49$.

- robustus $2: 48,49 ; 6-7: 42 ; 17: 35$.

_- f. hippophaës $5: 91 ; 8: 18 ; 10: 181$.

— salicinus 3: 53; 4: 28, 59; 5: 92.

— torulosus 17: 35 .

- tremulae 4: 44; 5: 91; 8: 18; 10: 181; 11: 31; 12: 47-49; 17: $33,35,84$.

Phellodon 6-7: 30, 31 .

- amicus 6-7: 31

- confluens 6-7: 31 .

- cyathiformis 6-7: 31 .

- graveolens 6-7: 31 .

- melaleucus 6-7: 31 .

- - var. ramosus 6-7: 31 .

— niger 6-7: $31 ; 17: 69$.

- tomentosus 6-7: $31 ; 14: 49,51$.

Phellorinia herculeana 17: 71 .

- inquinans 17: 71 .

- - var, herculeana 17: 71.

Phelonites minima 19: 12.

- strobilina 19: 12, 15.

Phlebia 6-7: 31.

- aurantiaca 6-7: 31.

- aurantiaca var. merismoides 5: 85 .

- - var. radiata 4: 43; 5: 85 .

- contorta 6-7: 31 .

- merismoides 5: 85 .

— radiata 4: 43; $5: 85$.

Phlebiella cadidissima 8: 18 .

- trachyspora 8: 18.

Phlegmacium 2: 61; 3: 48; 4: 36, 52, 60; 5: 51, 52; 10: 19, 23.

— alboviolaceum 4: 54; 5: 62; 6-7: 22 .

- allutum 3: 48; 4: 54; 5: 59.

- amarescens 5: 61 .

- amethystinum 5: 62 .

- amoenolens 4: 36; 5: 60

— anomalum 4: 54; 5: 62 .

— - var. lebretonii $4: 54 ; 5: 62,63 ; 6-7: 24$.

- - var. pineti 5: 62 .

— argentatum 4: $54 ; 5: 62$

- arquatum 3: 22; 5: 60 .
— aurantiacum 3: 59; 4: 36, 54; 5: 59; 6-7: 23.

- balteatum 3: 22, 28, 48; 5: 59; 6-7: 24.

- bolare 6-7: 24 .

- bulliardii 5: 62 .

- caesiocyaneum var. juranum 5: 60.

— calochroum 3: 66; 5: 60; 6-7: 23 .

- camphoratum 4: 54; 5: 62 .

- caninum 4: 54; 5: 62 .

- cedretorum 3: 66; 5: 61 .

- cephalixum 3: 22, 39, 48, 59; 4: 54; 5: 60; 6-7: 23 .

- cinereoviolaceum 4: 54; 5: 62.

— claricolor 3: 39, 48, 66; 5: 59 .

— cliduchum 3: 48, 59; 4: 54; 5: 60; 6-7: 23.

- coerulescens 2: 55 .

- compar 4: 37; 5: 60; 6-7: 23.

— crassum 3: 31, 59; 5: 59, 62; 6-7: 23, 24.

- crocolitum 2: 55.

- cyanopus $3: 39 ; 5: 60$.

- cumatile var. daulhoyanae 6-7: 23 ,

- cyanopus 4: 36; 6-7: 23.

- decolorans 3: 28, 39, 48, 59, 66; 5: 60; 6-7: 24.

— decoloratum 3: 39, 59; $4: 37,54 ; 5: 62 ; 6-7: 24$.

- - var. betuleti $3: 39,48$

- - var. betuli 5: $62 ; 6-7: 24$.

- diabolicum 6-7: 24.

- elegantior 3: 67; 5: 61

- eumorphum 6-7: 24.

- ferrugineum $3: 66 ; 4: 54 ; 5: 59 ; 6-7: 23$

- flavescentium 3: 66; 5: 61 .

— fulgens $2: 55 ; 3: 39,67 ; 4: 54 ; 5: 61 ; 6-7: 24$.

— fulmineum 3: 48, 59; 4: 54; 5: 61 .

- glaucopus 2: 55; 3: 22; 5: 60; 6-7: 23.

- hircinum 5: 60, 62 .

— infractum 3: 31, 39, 48, 67; 5: 61; 6-7: 24.

- kauffmanianum 5: 62 .

- largum 3: 58; 5: 60.

- latum 6-7: 23.

- lepidomyces 5: 62.

- lepidopus 5: 62.

- malachium 6-7: 24.

- melliolens 3: 48, 59 .

- minus 5: 59; 6-7: 23 .

- montanum ssp. europaeum $3: 31,39 ; 4: 36 ; 5: 61$.

- multiforme 2: 55; 3: 22, 28, 31, 39, 48, 59; 5: 59; 6-7: 23.

— - f. ochropallidum 5: 59.

- - var. rufescens 3: 48; 5: 59.

- napus $3: 59 ; 4: 54 ; 5: 59$.

- nemorense $3: 39,48 ; 5: 60 ; 6-7: 23,70$.

- obscurocyaneum $3 ; 31,39 ; 4: 37,54 ; 5: 61$.

- ochropallidum 3: 48 .

—odorifer 3: 66; 5: 61 .

- olidum 3: 22, 39; 4: 37, 54; 5: 60.

- olivascens $3: 22,59 ; 4: 54 ; 5: 61$.

- opimum 5: 62.

— orichalceum 3: $28,39,48,66 ; 5: 61$.

- papulosum 3: 48; 6-7: 23 .

- percome 3: 22; 5: 62 .

- persoonii 6-7: 24.

- pholideum 4: 54; $5: 62$.

- polychroum 5: 61 .

- porphyropus $3: 39 ; 4: 37,54 ; 5: 61$.

- pseudobolaris 5: 62 .

- pseudocrassum 3: 58; 4: 54; 5: 59.

- pseudonapus 5: 59; 6-7: 23.

— purpurascens 2: 55; 3: 52, 66; 5: 61; 6-7: 24

- - f. eumarginatum 4: 54.

- rapaceum f. majus (major) 3: 39, 48; 5: 59.

- - f. minor 6-7: 23. 
- rubicundulum 5: 62 .

- russeoides 5: 62 .

- resseum 5: 62.

- saginum 6-7: 23 .

- scaurum 3: 31, 39; 4: 36, 54; 5: 61.

- sebaceum 4: 54; $5: 59 ; 6-7: 23$.

- separium 4: 54; 6-7: 23.

- serarium 3: 48, 59; 5: 59.

— sericellum 4: 54; 5: 59.

- spilomeum 5: 62 .

— - var. depauperatum 5: 62.

- subarcuatum 5: 60.

- subatkinsonianum 5: 60.

- subbalteatum 6-7: 24.

- subpurpurascens 3: 39, 66; 5: 61 .

- subsimile 3: 31; 5: 61 .

- subtortum 5: 61; 6-7: 24.

- subtriumphans 3: 59, 66; 5: 60; 6-7: 23.

- sulphureum 3: 67; 5: 61.

-- tabulare 5: 62; 6-7: 24.

— talum 3: 39, 48; 5: 61 .

- traganum 4: 54; 5: 54, 62 .

- - var. finitimum 5: 62 .

- triumphans 2: 55; 3: 22, 28, 39, 48, 59; 4: 36, 60; 5: 60.

— f. minor $3: 59 ; 5: 60$.

— turmale 3: 48, 59; 4:54; 5: 59, 60; 6-7: 23.

— urbicum 5: 62; 6-7: 24 .

— validum 4: 36; 5: 60; 6-7: 23 .

- variecolor 5: 60 .

- - var. nemorense 3: 39; 5: 60 .

— varium 3: 28, 39; 5: 60 .

- - var. decolorans 5: 60; 6-7: 23.

— vespertinum 4: 36; 5: 59; 6-7: 23 .

— visitatum 4: 54; 5: 61; 6-7: 24.

- xanthocephalum 6-7: 24.

Phleospora aenigmatica 17: 1, 11, 16.

Pholiota 1: $18,20,31,42 ; 2: 56,60 ; 3: 59 ; 4: 37,55,60 ; 5: 49$, $65,101,103-105 ; 6-7: 19,24 ; 10: 178 ; 14: 114$.

— abstrusa 4: 55; 5: 66; 6-7: 24, 25.

- agardhii 6-7: 25.

- albocrenulata 8: 22, 23.

- alnicola $2: 61 ; 3: 22,28,40,49,59 ; 4: 37 ; 5: 41,65 ; 11: 31$; 12: 47-49; 13: 45.

— apicrea 5: 65 .

— astragalina $3: 38,49,59,67 ; 4: 37 ; 5: 65 ; 18$ (suppl.): 41 .

— aurivella $2: 61 ; 3: 67 ; 5: 65 ; 6-7: 24 ; 10: 181 ; 11: 23-26 ; 12$ : 47-49; $13: 45$.

- autumnalis 1: 23.

- caperata 2:61;3:7.

- carbonaria $2: 61 ; 3: 40,49 ; 4: 28,37,55 ; 5: 65,100-105$; 6-7: 24; 10: 180.

— - var. gigantea 4: 55 .

— confragosa $3: 7,31 ; 5: 65 ; 18: 55$.

- concissa 5: 65 .

- curvipes $4: 37 ; 5: 65$.

- decussata 4: 55: 5: 65 .

— filaris 4: 35 .

— flammans $2: 61 ; 3: 28,40,49,59,67 ; 4: 37 ; 5: 65 ; 10: 182$.

— flavida $2: 61 ; 3: 28,53,67 ; 4: 55 ; 5: 65$.

- fusca 5: 65 .

- gummosa 3: 28, 40, 67; 4: 37; 5: 65 .

- - f. ochrochlora $3: 67 ; 5: 65$.

- heteroclita $3: 28,31,59,67 ; 5: 65 ; 6-7: 24$.

— inaurata $4: 55 ; 5: 66 ; 6-7: 24$.

- lenta $2: 61 ; 3: 67 ; 4: 37,55 ; 5: 65 ; 6-7: 24 ; 10: 180 ; 11: 26$.

- lubrica 3: 28, 53, 59; 4: 37, 55; $5: 65 ; 10: 180$.

- marginata $1: 31,42 ; 2: 61$.

- mixta 5: 65 .
- mutabilis 1: 18, 88; 2: 49, 61; 3: 7-9; 9: 40; 10: 181; 18(suppl.): 75.

- myosotis $2: 61 ; 4: 37,55 ; 5: 66$.

- -f. minor 3: 31; 4: 55; 4: 55; 5: 66.

- praecox 2: 61 .

- pumila 3: 40 .

- scamba 3: 49, 59; 5: 66.

- spumosa $2: 61 ; 3: 22,28,49,59 ; 4: 37,55 ; 5: 65,66 ; 10$ : 180.

— squarrosa $2: 61 ; 3: 28,67 ; 5: 65 ; 6-7: 42 ; 8: 47,48,59 ; 10$ : $181 ; 11: 26,31 ; 12: 48,49 ; 13: 45 ; 18$ (suppl.): 116, 117.

- squarrosoadiposa 5: 65 .

- subsquarrosa 3: 67; 6-7: 24.

— tuberculosa 3: 40, 49; 4: 37, 55; 5: 65.

— vahlii 5: 39.

Pholiotina 4: 55; 5: 64; 6-7: 18.

— appendiculata $3: 48 ; 5: 64$.

- blattaria $4: 37,54 ; 5: 63 ; 6-7: 24$.

- brunnea 4: 55; 5: 64.

- coprophila 5: 63.

- exannulata 5: 64.

- filaris 3: 28, 31; 4: 54; 5: 63; 6-7: 24.

— intermedia var. brunnea 5: 64 .

- subnuda 5: 63.

- togularis $3: 28 ; 5: 63$.

— - var. filaris 3: 28, 31; 5: 63 .

- f. teneroides 5: 63 .

- teneroides 3: 28, 48; 4 : 37.

Phragmidium potentillae 6-7: 95 .

Phycomyces 18(suppl.): 74.

- blakesleeanus 12: 55, 57

Phylacteria laciniata 3: 29.

- palmata 2: 29, 51, 67; 5: 88 .

- terrestris 3: 29, 32, 42, 51, 61, 67; 4: 40, 59; 5: 88.

Phyllachora 15: 47.

— agrostidis 15: 59.

- gangraena 15: 56.

- graminis 15: 46-49, 59.

- silvatica (sylvatica) 15: 46-49.

Phyllactinia antarctica 17: 14.

- clavariiformis 17: 14.

- suffulta 4: 21 .

Phylloporus 18: 16 .

Phyllosticta coriarii 17: 15 .

Phyllotopsis nidulans 5: 22; 10: 181; 12: 48, 49, 63.

Physarum 18: 60.

- albipes 19: 12, 13.

- album 19: 8.

- apiculosporum 18: 24, 25, 58; 19: 12, 15.

— atrum 14: 58; 19: 12, 16.

— aureum 14: 57.

- auriscalpium 14: 57; 19: 12.

- bitectum 14: 60 .

- bivalve 14: 59; 19: 10,12, 13 .

— cinereum 14: 58, 59, 79; 17: 28; 19: 12.

- citrinum 14: 57; 19: 12, 13, 16.

- compressum 14: 58, 78; 19: 12.

- confertum 19: 12,16

— conglomeratum 14: 59; 19: 12.

- connatum 14: 58; 19: 12.

- contextum 14: 59; 19: 12.

- - var. splendens 19: 12.

- decipiens 19: 10, 12.

- diderma 14: 60, 78; 19: 12.

- didermoides 14: 58, 78; 19: 12, 16.

- - var. lividum 14: 60.

- globuliferum 14: 57; 19: 12.

- lateritium 19: 1, 6, 12 . 
- leucophaeum 14: 58; 19: 12.

- leucopus 14: 56; 19: 12.

- lilacinum 19: 1 .

— maydis 14: 57, 78; 17: 26; 19: 12, 13.

- melaleucum 19: 10, 12, 14.

- notabile 19: 12.

- nudum 19: 12.

- nutans 6-7: 110; 14: 58, 79; 17: 31; 19: 9, 12, 13.

— - var. leucophaeum 14: 58; 19: 12.

—oblatum 14: 57; 17: 26; 19: 12, 13.

— ovisporum 18: 24-26.

— psittacium 6-7: 110; 14: 57, 78; 19: 13.

— pusillum 17: 19, 28; 19: 13.

- robustum 19: 12, 13.

- rubiginosum 14: 59, 79; 19: 13 .

- schroeteri 19: 13, 16,

- schumacheri 19: 12, 13.

- sinuosum 14: 59; 19: 12, 13.

— straminipes 14: 57; 19: 13.

- thejoteum 19: 13.

- utriculare 19: 10, 13.

- - var. melaleucum 19: 10, 13, 14.

— vernum 14: 58, 59; 18: 24.

— virescens 14: 59; 19: 13.

_- _ var. nitens 14: 60 .

— - var. obscurum 14: 60 .

— viride 6-7: 110; 14: 57; 17: 26; 19: 13.

— - var. aurantiacum 14: 57; 19: 13.

- - var. aureum 14: 57.

_- var. incanum 14: 57; 19: 13.

Physisporus xantha 5: 93.

- vulgaris 3: 42.

Physomitra infula 9: 13.

- - var. friesiana 9: 13 .

Phytophthora erythroseptica 12: 55 .

Piptoporus betulinus 8: 18; 10: 181; 11: 26, 31; 12: 47-50; 13: 44-46.

- pseudobetulinus 20: 6 .

- quercinus 17: 36 .

Pisolithus 10: 170.

- tinctorius 10: 170, 174.

Pithya 14: 124.

Plasmopara crustosa 8: 6.

- nivea 17: 3,4 .

- pusilla 8: 6 .

Nectania 6-7: 105, 106; 17: 41.

- hiemalis 4: 27; 5: 97.

- protracta 4: 27; 5: 97; 6-7: 105, 106.

- - var. winteri 6-7: 105, 106.

Pleospora herbarum 17: 3, 14.

- phaeocomea 12: 23 .

Pleurocybella 2: 62; 10: 10 .

- lignatilis 2: $62 ; 5: 15$.

- porrigens 2: 62 .

- tessulata 6-7: 8 .

Pleurodon 1: 35 .

— auriscalpium 3: 29, 42, 51; 4: 40; 5: 87.

Pleurotellus acerosus 4: 61; 5: 39.

- mutilus 5: 30, 39 .

- planus 5: 39.

— pubescens $4: 33 ; 5: 39$.

- septicus 4: 33; 5: 39 .

- tremulus $3: 65 ; 5: 39$.

Pleurotus $1: 30,42 ; 5: 22 ; 10: 115 ; 15: 24 ; 18$ (suppl.): 10, 17 , $18,53,54,60,70,73,75$.

- acerosus 3: 34 .

- cornucopiae $3: 45 ; 5: 22 ; 18$ (suppl.): 17.

- cornucopioides 5: 22 .
- corticatus $2: 62 ; 5: 22 ; 17: 65$.

- - var. tephrotrichus 3: 64 .

— dryinus $2: 62 ; 3: 64 ; 5: 22 ; 10: 115 ; 12: 48,49 ; 17: 65$.

- eryngii 17: 59,65 .

- florida 18(suppl.): 53.

- lignatilis 2: 62 .

- limpidoides 5: 23.

- mitis 2: 62; 10: 182; 12: 63 .

— ostreatus $2: 62 ; 3: 26,34 ; 5: 22 ; 10: 181 ; 11: 31 ; 12: 47-50$; 13: 46; 16: 10, 12, 13; 18(suppl.): 17, 18, 29, 30, 33, 34, $43-45,53,59,70,75,116,117$

- - var. columbinus 6-7: 10.

- - var. pulmonarius 4: $31 ; 5: 22$.

- - var. salignus 5: 22 .

- porrigens 2: 62 .

- pulmonarius 4: 31 .

- - var. ostreatus 3: 34 .

- salignus $3: 26 ; 4: 31,42,47$.

- sapidus $3: 45 ; 5: 22$.

- serotinus 2: 62 .

- tephrotrichus 3: 64 .

- ulmarius 2: 62; 3: 25 .

- viscidus 18: 29, 30 .

Plicaria 6-7: 34.

- assimilata 6-7: 34 .

— badia 3: 24, 30, 32, 43, 62; 4: 41, 59:5:96, 97.

- brunneoatra 3: 32, 62; 5: 96.

- coronaria 1: 36 .

- echinospora 4: 59; 5: 96.

- fimeti 3: 62; 4: 59; 5: 97.

- macrospora 5: 97.

- pustulata 6-7: 34 .

— repanda $3: 24,30,43 ; 5: 97 ; 6-7: 34$.

- stevensonia 4: 59; 6-7: 34 .

— varia $3: 24,43 ; 4: 59 ; 5: 97 ; 6-7: 34$.

— vesiculosa $3: 30,43 ; 4: 41 ; 5: 97$.

Plicatura nivea 4: 58; 5: 86; 6-7: 31 .

Pluteus 1: 30, 42; 3: 64; 4: 33, 48, 49; $5: 34,35$.

— atromarginatus $3: 31,56 ; 4: 33 ; 5: 34$.

- carneipes 4: $33 ; 5: 34$.

- cervinus $1: 25 ; 2: 62 ; 3: 21,27,31,35,46,56,64,65 ; 4: 33$, 48; $5: 34 ; 6-7: 13$.

- chrysophaeus $5: 35$.

— cinereus $4: 48 ; 5: 35 ; 6-7: 13,14$.

- cyanopus 5: 35 .

- ephebeus 6-7: 13 .

- hispidulus 4: $33 ; 5: 34$.

- leoninus 3: 64; 5 : 35 .

- luteomarginatus 5: 34 .

- lutescens 5: 35.

- minutissimus f. major $3: 56 ; 5: 35 ; 6-7: 14$

- murinus $3: 64 ; 4: 33 ; 5: 34 ; 6-7: 13$.

- nanus $3: 21: 5: 35$.

- - var. lutescens 5: 35 .

— nigroflocculosus $3: 31 ; 4: 33 ; 5: 34$.

— patricius $4: 33 ; 5: 34$

- pellitus 3: $35 ; 4: 33 ; 5: 34,35$.

— petasatus 1: 30, 88; 3: 64; 4: 33; $5: 34,35 ; 6-7: 13$.

— phlebophorus 4: 33; $5: 35 ; 6-7: 14$.

- plautus $4: 49 ; 5: 35$.

- pseudorobertii 4: 33; 5: 35 .

— robertii 4: $33 ; 5: 34$.

- roseipes $4: 33,48 ; 5: 34$.

— salicinus 4: 33; 5: 34; 6-7: 13 .

- umbrosus $3: 21,31,46 ; 5: 34,35$.

- villosus 6-7: 13.

Podaxis pistillaris 17: 59,71 .

Podofomes trogii 17: 33, 36, 38. 
Podoporia sanguinolenta 8: 19 .

Podosphaera aucupariae 4: 21.

- leucotricha $4: 21 ; 17: 14$.

- major 4: 21; 8: 6 .

- myrtillina $4: 21 ; 8: 6,7$.

- oxyacanthae $4: 21$.

— tridactyla 4: 22.

Podostroma 15: 28.

Polypilus frondosus 6-7: 35; 8: 9; 10: 181; 11: 31; 12: 47-50.

— umbellatus 8: 10.

Polyporus 1: 7, 10, 35; 5: 91; 10: 178; 12: 65; 13: 6; $20: 8$.

— adustus 8: 15 .

- amorphus 8: 17.

- anisoporus 17: 38 .

— arcularius 8: 19; 17: 38 .

- $\mathrm{f}$. griseus 8: 19 .

- aurantiacus 20: $1,8,10,12,13$.

- betulinus $2: 49 ; 3: 8$

— brumalis 8: 19; 10: 181; 11: 31; 12: 47-50, 63; 13: 45, 46.

— ciliatus 14: 51 .

- cinnamomeus 8: 15.

- confluens $3: 24 ; 4: 59: 5: 88 ; 6-7: 31$.

- corni 17: 77, 84 .

- fibrillosus 20: 1,8 .

- frondosus 2: 48-50; 6-7: 43, 68 .

- fulvus 17: 80

- hirsutus 12: 63 .

— igniarius 8: 18.

- - ssp. pomaceus 8: 18 .

- leucomelas 3: 53; 5: 88 .

- lithuanicus 20: 8 .

- mollis 3: 29; 5: 91 .

— nidulans 8: 17 .

- ovinus $3: 7,8,23,29,32,42,51,61 ; 4: 42,60 ; 5: 88 ; 8: 19$, $37,39,47,48,51,58-60$.

— perennis 6-7: 37 .

— pinicola 3: 24; 8: 16 .

- pomaceus 17: 77 .

- populinus 8: 17 .

- radiatus $8: 17$

- semisanguinus 18: 48.

- shiraianus 20: 8,12 .

- squamosus $6-7: 71 ; 8: 19 ; 10: 181 ; 11: 23,25,26 ; 13: 6$; 18(suppl.): 25-27.

- stipticus 3: 51; 5: 91; 8: 19.

— sulphureus $2: 48-50 ; 3: 51,53 ; 5: 88 ; 6-7: 42,72 ; 8: 17$.

- $\mathrm{f}$. aporeus 5: 88 .

- tomentosus 8: 19; 18(suppl.): 50

— varius 8: 19; 10: 181 .

Polystictus 1: 69 .

- circinatus 11: 31

- - var. triqueter 10: 182

- perennis 8: 15 .

- tomentosus 8: 19.

- versicolor 1: 61, 68-71.

Polystigma ochraceum 4: 22.

Poria 1: 68; 4: 41; 5: 92, 93; 18: 48 .

— argentea 5: 90 .

- armeniaca 5: 90

— caesioalba 5: 90

- calcea 3: 30; 5: 93.

_ - var. fragilis 5: 93.

- callosa 5: 91 .

- crassa 18: 47, 48.

- crustulina 20: 6 .

- eupora 5: 93

- greschikii 18: 47.

- inconstans 5: 92.
- lindbladii 18: 48

- mollusca 5: 93.

— mucida 5: 93.

—obliqua $4: 44 ; 5: 92 ; 8: 17 ; 13: 45$.

— placenta 20: 6,7 .

- rixosa 5: 93 .

- romellii 18: 48 .

- taxicola 5: 92

— vaporaria 1: 61, 68-70; 5: 93; 8: 16; 12;63.

- versipora 6-7: 42

— violacea 5: 90 .

- viridans 5: 92

— vulgaris 3: 42; 5: 93 .

- xantha $4: 44 ; 5: 93 ; 8: 14 ; 18: 48$

Psalliota 1: $31 ; 2: 62 ; 5: 117 ; 17: 60$.

- abruptibulba 1: 85 .

— amethystina 17: 60 .

- augusta 3: 7 .

- flavescens 1: 31 .

- hortensis 3: 5 .

- rubella 17: 60 .

- silvicola 3: 7 .

- xanthoderma 1: 21, 31 .

Psathyra 1: 32, 43; 2: 62 .

Psathyrella 1: 32, 43; 2: 57, 62; 4: 60; 5: 70, 73; 6-7: 26, 27.

- agaves 17: 59,62

— albidula 5: 70; 6-7: 26 .

- - var. palustris 5: 70

— appendiculata $3: 53 ; 5: 72$.

- atomata $3: 40 ; 4: 38 ; 5: 70 ; 6-7: 26$.

— badiophylla 5: 71 .

- candolleana $2: 62 ; 3: 22,28,31,40,49,60 ; 4: 39,57 ; 5: 72$; 17: 62 .

- $\operatorname{casca} 4: 57 ; 5: 71$

- cernua 5: 73:6-7: 26 .

- chondroderma $5: 72$

- confertissima 6-7: 26.

- conopilea 5: 71; 6-7: 26 .

- consimilis 5: 73 .

- coprobia $4: 38,56 ; 5: 71 ; 6-7: 26$

- corrugis $3: 22.28,40 ; 5: 70,71$.

- cortinarioides 17: 62 .

— egenula $4: 39,57 ; 5: 72$.

— fatua $2: 62 ; 5: 72 ; 6-7: 27$

- fasciculare 5: 72 .

— fasciculata 4: 57; 6-7: 26.

- fibrillosa 4: 57; 5: 72 .

- fimicola 5: 71; 6-7: 26

- fragilissima 4: 38 .

- frustulenta $4: 57: 5: 71$.

- fusca 5: 72; 6-7: 27.

- gordonii 5: 71.

— gossypina 4: 56; 5: 71; 6-7: 26 .

— gracilis $3: 28 ; 4: 38 ; 5: 70,71 ; 10: 180$

— - var. corrugis $3: 22,40 ; 4: 38 ; 5: 71$.

— gyroflexa $3: 60 ; 4: 39,58 ; 5: 72 ; 6-7: 27$

- hydrophila $3: 28,53 ; 4: 39 ; 5: 72$.

- hypsipoda 6-7: 26.

— infida 4: 38; 5: 71.

- lacrymabunda $3: 28 ; 5: 73$.

- lactea 4: 39.

- lepidota 6-7: 27.

- leucotephra 6-7: 26.

- longicauda 5: 70; 6-7: 26 .

- marcescibilis $4: 38 ; 5: 72$.

- melanthina 6-7: 27; 17:62.

- microrhiza 5: 70 .

— f. polycystis $3: 67 ; 5: 70$. 
- f. pseudobifrons 5: 70.

- multipedata 6-7: 26.

- noli-tangere $4: 56,57 ; 5: 71 ; 6-7: 26$.

— obtusata $3: 28,60,67 ; 4: 39,57 ; 5: 72,73 ; 6-7: 27$.

— orbitarum 5: 71 .

— papyracea 5: 73 .

- pennata 2: 62 .

- populina 6-7: 27.

- prona $3: 22 ; 4: 38 ; 5: 71 ; 17: 62$.

- - var, utriformis 17: 62 .

- pseudocasca 4: 57; 5: 71.

— pseudogracilis 5: 70 .

— pygmaea 5: 73 .

- reticulata 6-7: 26, 27 .

- sarcocephala 5: 73.

- silvestris 6-7: 27.

— spadicea $3: 28,60,67 ; 5: 73 ; 6-7: 42 ; 11: 26 ; 12: 47-49$.

— spadiceogrisea $3: 31,49 ; 4: 39 ; 5: 72 ; 17: 62$.

- - var phaeophylla $5: 72$.

— sphagnicola 4: 56; 5: 71; 6-7: 26.

- spintrigera 4: 28, 39; 5: 71 .

- stercoraria 5: 71 .

- stipatissima 5: 72; 6-7: 26.

- subatomata 4: 38, 56; 5: 70 .

- subatrata 5: 71; 6-7: 26 .

— subnuda $3: 40,60 ; 4: 39,57 ; 5: 72 ; 6-7: 27$.

- subpapillata 3: 28; 5: 72 .

— velutina $3: 28 ; 4: 38 ; 5: 73$.

- vernalis 5: 72 .

- f. gracillima 5: 73 .

- vinosofulva 17: 62.

Pseudoclitocybe 10: 9-11; 14: 87, 126, 128; 15: 17.

- atra 14: 126-128; 15: 16, 17.

- cyathiformis 14: 126, 128; 15: 16, 17.

Pseudocoprinus 1: 32,43 .

- disseminatus 2: 62; 3: 22, 49.

Pseudodiplodia 19: 31 .

- cenangiicola 19: 30,31 .

Pseudohiatula $2: 30,62 ; 3: 26 ; 4: 31 ; 5: 24 ; 6-7: 11 ; 17: 68$.

— conigena $2: 29 ; 3: 54,55 ; 4: 31 ; 5: 24$.

— esculenta 2: 29, 59; 3: 20, 30, 34, 54 .

- - ssp. pini 2: 29.

- favrei 2: 30 .

- stephanocystis 3: $55 ; 4: 28 ; 5: 24$.

— tenacella $2: 28-30,45,55,59 ; 4: 27,28,44,47 ; 5: 24 ; 6-7$ : 11.

Pseudomarasmius 6-7: 18 .

Pseudombrophila 14: 125.

Pseudomonas 18(suppl.): 74, 86; 19: 58.

- putida 18(suppl.): 75.

Pseudopeziza singularis 17: $3,14$.

Pseudoplectania 14: 124.

— nigrella 5: 97.

Pseudorhizina 9: 9; 13: 48-50, 55; 14: 109-112, 125, 141; 16: 6.

- californica 13: 48, 56; 14: 111 .

- sphaerospora 9: 9, 10; 13: 48, 49, 54

Pseudorhizinaceae 14: 109 .

Psilocybe 1: 32,$43 ; 2: 49,61,63 ; 14: 50 ; 17: 63 ; 18$ (suppl.): 6.

- atrobrunnea 4: 38, 55; 5:67; 6-7: 25 .

- coprobia 4: 55 .

- coprophila 3: 22, 40, 49, 59; 4: 38; 5: 67; 6-7: 25.

- var, subcoprophila 6-7: 25 .

- elongata 2: 62 .

- foenisecii 2: 62 .

- merdaria 3: 40, 49, 59; 4: 38, 55; 5: 67.

- polytrichi 5: 66 .

— semilanceata 2: 62: 3: 53, 59; 5: 67 .
— spadicea 3: 28.

— turficola 4: 55: 5: 67 .

—uda $2: 62 ; 3: 59 ; 4: 37 ; 5: 66 ; 6-7: 25$.

- - f. sphagnicola 3: 40 .

Puccinia 17: 7.

- absinthii 4: 15 .

- acetosae 8: 7 .

- actaeae-agropyri 15: 47.

- andina 17: 7 .

- arenariae 6-7: 95.

- brachypodii 17: 16.

- - var. arrhenatheri 17: 7, 10, 13.

_ - var. major 17: 7.

- - var. poae-nemoralis 17: 10 .

- calcitrapae 8: 7.

- callaquensis 17: 7 .

— caricina 6-7: 95; 17: 3, 7, 14.

- - var. uliginosa 8: 7 .

- - var. urticae-acutae 8: 7 .

- caricis-bonariensis 17: 8 .

- caricis-gayanae 17: 7 .

- chilensis 17: 7 .

- chrysosplenii 6-7: 95 .

- cnici 17: 3, 7 .

- coronata 6-7: 96; 8: 7; 15:41; 17: 7, 10.

- - var. arrhenatheri 17: 10

- cryptica 17: 3,8 .

- cynoctoni 17: 8 .

- cynodontis 17: 8, 14.

- deschampsiae 8: 5,7 .

— dioicae 6-7: 96; 8: 7; 17: 8 .

- eleocharidis 17: 8 .

- epilobii-tetragonii 17: 10 .

— fergussonii 8: 7 .

- gnaphaliicola 17: 8 .

— graminella 17: 8 .

- graminis $8: 5 ; 17: 8$.

- helianthi 17: 8 .

- hieraccii 17: 3, 8 .

— - var. hieracii 8: 7 .

- - var. piloselloidarum 8: 7

- hieraciiphila 17: 8 .

- hordei 17: 8.

- hydrocotyles 17: 8 .

— iridis 6-7: 96.

- komarowii 2: 43-45.

- liberta 17: 3, 8

- luzulae 6-7: 96.

- luzulicola 17: 8 .

- lyciicola 17: 8 .

- malvacearum 6-7: 102, 103; 17: 8 .

- menthae 17: 8 .

- meyeri-alberti 17: 8, 12, 15.

- modiolae 17: 8

— nuda 17: 8 .

— oblonga 6-7: 96.

- obscura 6-7: 96;8: 7; 17:3,8, 14.

- paspali 17: 8 .

- perforans 17: 8 .

- philippii 17: 10.

- pimpinellae 4: 19.

- poae-nemoralis $8: 5,7 ; 17: 3,10,14$.

- poarum 8: 7 .

- polygoni-amphibii 6-7: 96, 97.

- praeandina 17: 10.

— pulverulenta 6-7: 97; 17: 10.

- punctata 8: 7: 17:3,10.

- recondita 8: 7; 17: 10 . 
- setariae 17: 10.

- singularis 6-7: 97.

- striiformis 17: 3, 10 .

- subnitens 17: 10, 14.

- taraxaci 4: 22.

- thalassica 17: 10

- thlaspeos 8: 7 .

- tumidipes 17: 8 .

- unciniarum 17: 10, 14.

— vaginatae 8: 7 .

- veratri 2: 47 .

- violae 17: 10, 15

Pucciniastrum areolatum 19: 15.

— padi 19: 15

— vaccinii 4: $21 ; 8: 7$.

Pulparia 13: 52; 14: 125.

Pulveroboletus lignicola 18: 10 .

- sulphureus 6-7: 55

Pulvinula 14: 125.

Pustularia 14: 138.

- catinus 13: 55.

Pustulina catinus 14: 106.

Pycnocalyx 2: 38 .

- abietis 2: $35,36,38$.

Pycnoporellus 20: 1, 2, 8 .

— alboluteus 11: 33; 20: 1-8.

- fibrillosus 20: 1, 8, 10, 12 .

- fulgens 20: 1, 5, 6, 8-14.

Pycnoporus cinnabarinus 8: 19; 10: 181, 182; 11: 26, 31; 12: $47-49 ; 13: 45 ; 20: 13$.

Pycnostysanus 14: 133, 135.

- resinae 14: 135.

Pyrenophora dactylidis 12: 23.

- tritici-repentis 12: 24-26, 33.

Pyricularia oryzae $14: 5,7$.

Pyronema 5: 100; 12: 61; 14: 109, 125.

- confluens 5: 104

- omphalodes 5: 100.

Radulum 4: 43.

- membranaceum 4: 43, 59, 61; 5: 86 .

— orbiculare 4: 43, 59; 5: 85, 86 .

- quercinum 5: 86; 6-7: 29, 30.

Ramaria 18(suppl.): 116, 117.

- aurea 17: 69.

- flava 16: 10, 12, 13; 17: 69; 18(suppl.): 43, 115, 117.

- formosa 17: 69 .

- mairei 17: 69 .

Ramularia 17: 15.

- alborosella 17: 15.

- aequivoca 17: 3,15 .

- biflorae 17: 3,15 .

— plantaginea 17: 3, 15 .

- pulchella 15: 38 .

- pusilla 15: 38 .

- repentis 17: 3,15 .

- urticae 17: 15.

Ravenelia papillosa 17: 10, 14.

Reticularia 14: 72; 19: 5, 15.

- atra 10: 13 .

— intermedia 14: 72, 78: 19:11, 13.

- jurana 19: 1, 5.

- lycoperdon 14: 71; 19: 5, 11, 13.

- - var. jurana 19: 5 .

- muscorum 19: 11,13.

- olivacea 19: 11, 13.

- splendens 19: 5.

_ - var. jurana 19: 5

- umbrina 19: 11, 13.
- versicolor 19: 11, 13.

Rhacodium resinae 14: 135.

Rhizina 9: 11; 13: 49; 14: 111, 125; 15: 33.

— inflata $3: 52,53 ; 5: 96 ; 6-7: 34$

- undulata 3: 52: 5: 96; 9: 11; 13: 54, 55.

Rhizoctonia solani 18(suppl.): 75.

Rhizomorpha subcuticularis 18(suppl.): 49.

- subterranea 18(suppl.): 49.

Rhizopogon 10: 170, 180; 18(suppl.): 93.

- luteolus 3: 23, 29, 50; 5: 93; 10: 174.

- roseolus 5: 93; 10: 175; 18(suppl.): 93.

— rubescens $3: 23,29 ; 5: 93 ; 6-7: 32$.

- - var. roseolus 3: 23 .

Rhizopus 9: 46.

- oligosporus 12: 55.

- suinus 14: 5 .

Rhodocybe 5: 29; 6-7: 12; 14: 87, 89, 90, 121, 122; 18: 22-24.

— caelata 5: 29; 10: 114

- fallax 14: 122.

- harperi 18: 29, 30; 19: 22.

- mundula 17: 59,68.

- nitellina 5: 29 .

— piperita (piperata) 14: 121, 122; 19: 24.

— smithii 14: 121, 122; 19: 24 .

- subharperi nom. prov. 19: 23.

— truncata 5: 29; 6-7: 12; 14: 12; 17: 59, 68; 19; 24.

Rhodopaxillus 14: 82 .

- cyclophilus 6-7: 9

- densifolius 14: 83 .

Rhodophyllus 1: 30,$42 ; 2: 56,59,60 ; 4: 48 ; 5: 30,32,33 ; 6-7$ : $13 ; 14: 50,52,89$.

- aemulans $3: 35 ; 5: 33$.

— ameides 4: 48; 5: 30; 6-7: 13 .

— anatinus $3: 46 ; 4: 32,48 ; 5: 32$

— aprilis $3: 34,55 ; 4: 28,44 ; 5: 30$.

_ - f. majalis 5 : 30 .

— $\mathrm{f}$. majus $4: 28,48 ; 5: 30$.

- $\operatorname{ardosiacus} 5: 33$.

- asprellus $2: 62 ; 3: 21,35,46,56 ; 4: 32,48 ; 5: 33$.

— atrides $4: 48 ; 5: 33$.

- byssisedus 5: 33; 6-7: 13 .

- caelestinus 4: 32; 5: 33 .

- carneoalbus $3: 56,64 ; 5: 33$.

— cetratus $2: 62 ; 3: 7,56 ; 4: 48 ; 5: 32 ; 6-7: 13 ; 18$ (suppl.): 41.

- chloropolius $3: 56 ; 5: 33 ; 6-7: 13$.

- clandestinus $3: 55,64 ; 4: 48 ; 5: 31$.

- clypeatus $2: 62: 3: 21,27,31,34,45,55 ; 4: 48 ; 5: 30$.

- cocles 6-7: 13 .

- $\mathrm{f}$. tridentinus 6-7: 13 .

— cordae $3: 45 ; 4: 48 ; 5: 31$.

- costatus $3: 27,34 ; 4: 48 ; 5: 31$.

- cyanulus 5: 33 .

- dysthales 4: 61: 5: 31 .

- elaphinus 6-7: 13 .

- - var. radiatus 6-7: 12 .

- erophilus 6-7: 12

- euchrous 5: 33; 6-7: 13 .

- fumosellus $4: 61 ; 5: 31$.

— griseocyaneus $2: 62 ; 3: 21 ; 4: 48 ; 5: 33$.

— griseorubellus $3: 21,35 ; 4: 32,42 ; 5: 32 ; 6-7: 13$.

- griseorubidus $5: 32$.

— hebes $3: 45: 4: 32: 5: 31 ; 6-7: 13$.

- hirtipes $3: 27,34,45 ; 4: 28,32,48 ; 5: 31$.

— icterinus $3: 56,64 ; 5: 32 ; 6-7: 13 ; 15: 27$.

- incanus 6-7: 13 .

— infulus $3: 46,56,64 ; 4: 48 ; 5: 32 ; 6-7: 13$.

- jubatus $3: 27.34: 5: 30$

- junceus $3: 27,35,46,56,64 ; 4: 32,48: 5: 31$. 
- lampropus $2: 63 ; 3: 21,35,46,56 ; 4: 48 ; 5: 32,33$.

- lanicus 17: 59,63.

- lazulinus 6-7: 13 .

- limosus 3: 35, 52, 64: 4: 48: 5: 32 .

- lividus $1: 25,30,36,42,46 ; 2: 63 ; 6-7: 52,59-61,69,70$, 73.

- majalis $3: 34,55 ; 4: 28,48$.

- mammosus $3: 27,34,45,55,64 ; 4: 32: 5: 31$.

— - var. sericoides $3: 55 ; 4: 32 ; 5: 31$.

- minutus $3: 21,35,52,56 ; 4: 48 ; 5: 33$

- mougeotii 5: 33 .

— nidorosus 1: $30,36,42 ; 3: 21,27,34,45,55 ; 4: 32,48 ; 5$ : 30; 6-7: 70 .

- nigrellus 4: $32: 5: 33$.

- nitidus 5: 30 .

- obsoletus 5: 31 .

_ papillatus $2: 63 ; 3: 55,64 ; 5: 31 ; 6-7: 13$.

— parkensis 4: 32,$48 ; 5: 33$.

- placidus $3: 35: 4: 48 ; 5: 32,33 ; 6-7: 13$.

- var. gracillis $3: 56 ; 5: 32$.

- politus 4: $32: 5: 30$.

- porphyrophaeus 6-7: 12.

- proletarius $5: 31 ; 6-7: 13$.

- prunuloides $3: 55 ; 5: 30 ; 6-7: 12$.

- radiatus 5: 30;6-7: 12 .

- repandus $3: 55 ; 5: 30$.

— rhodocylix $3: 35 ; 4: 32 ; 5: 33$.

— rhodopolius $1: 30,36,42,46: 2: 63 ; 3: 21,27,34,45,55 ; 4$ : 32,$48 ; 5: 30 ; 6-7: 13$.

- rhombisporus $5: 32$.

- rickenii 4: $32 ; 5: 31 ; 6-7: 13$.

- rusticoides 3: 46; $4: 32 ; 5: 33$.

- sarcitulus 4: 48 .

- - var. majusculus 6-7: 13.

- - var. spurcifolius $3: 64 ; 5: 33 ; 6-7: 13$.

— sarcitus $3: 64 ; 4: 48 ; 5: 33 ; 6-7: 13$.

- saundersii 5: 30 .

- sericellus $2: 63 ; 3: 56,64 ; 4: 48 ; 5: 33$.

- - var. lutescens 5: 33 .

- sericeus $2: 63 ; 3: 21,27,35,46 ; 4: 32,48 ; 5: 31 ; 17: 63$.

- serrulatus $2: 63 ; 3: 21,46 ; 4: 32,48 ; 5: 33 ; 6-7: 13$.

- solstitialis $5: 32 ; 6-7: 13$.

- speculus $3: 55,64 ; 4: 48 ; 5: 30$.

— staurosporus $2: 63 ; 3: 21,27,34,45,55 ; 4: 32,48 ; 5: 31$.

- var. platyphyllus 6-7: 13 .

_ - var. rickenii 3: 64; 4: 48; 5: 31 .

— turbidus 3: 27; 4: 32; 5: 30 .

- turcii 5: 32 .

- undatus $2: 63 ; 3: 64 ; 5: 33 ; 6-7: 13$.

- - var. viarum 6-7: 13; 17:63.

- vernus 4: 44:5: 30 .

- xylophilus 5: 31 .

Rhodotorula rubra 12: 65 .

Rhynchosporium 12: 14; 13: 23-25, 27, 30.

- graminicola 13: 23 .

- orthosporum 12: 14; 13: 23-30.

- secalis 13: 23-25, 27-30.

Rhytisma acerinum $3: 30 ; 5: 98$.

Ripartites 3: 59; 5: 18; 6-7: 9, 15; 14: 89 .

- albidoincarnatus $3: 65 ; 4: 33 ; 5: 39$.

- helomorphus $3: 46,56,65 ; 5: 15,39 ; 6-7: 15$.

- strigiceps 6-7: 15.

- tricholoma 3: 27, 46, 56; 5: 39;6-7: 15 .

Rigidoporus ulmarius 17: 38 .

Rozites 2: 61 .

— caperatus (-a) $2: 63 ; 3: 7,8,21,27,31,35,46,56 ; 4: 34,49$; $5: 40 ; 6-7: 37,70 ; 8: 36,37,47,48,57,59 ; 14: 50$; 18(suppl.): $6,41,108,115,117$.
Russula 1: $21,24,26,34,36,39,44,46 ; 3: 6,8,9,14,18,19$, $44 ; 5: 5,6,76,78 ; 6-7: 30,68 ; 8: 37,38,46,48,59: 9: 36$; 10: 169; 14: 51; 18(suppl.): 49, 51, 108-111, 114, 116, 117. - adulterina 17: 59, 66 .

— adusta $2: 63 ; 3: 23,28,31,41,49,60 ; 4: 57 ; 5: 75$.

- aeruginea 1: $37: 2: 63 ; 3: 8,23,28,32,41,49,60: 4: 40,57$; 5: 76; 6-7: 37; 18(suppl.): 39, 116, 117 .

- albonigra $3: 23 ; 5: 75 ; 6-7: 27$.

- alutacea $1: 34 ; 3: 32,49,60,67 ; 4: 40,57 ; 5: 76 ; 18$ (suppl.): $102,104$.

- amethystina $5: 76 ; 6-7: 28$.

- amoena 6-7: 27.

- anomala 4: $58: 5: 78$.

- atropurpurea $3: 32,67 ; 4: 40,43,58 ; 5: 77$.

- - var. depallens 4: 62; 5: 77 .

— aurata $3: 23,41 ; 5: 77 ; 6-7: 28 ; 8: 12$.

- azurea 3: 49; 5: 76 .

- badia $1: 25,34 ; 6-7: 28$.

- betulina 4: $58: 5: 77$.

- blackfordiae 5: 78; 6-7: 27.

- brunneoviolacea $3: 60 ; 5: 76$.

- caerulea $3: 32 ; 5: 76: 6-7: 28$.

— - var. umbonata $3: 32: 5: 76 ; 6-7: 28$.

- cessans 4: 40 .

- chamaeleontina $3: 23,32,41,49,60 ; 4: 40,57 ; 5: 77$.

- cicatricata 17:67.

- claroflava $2: 63 ; 3: 8,23,28,32,41,49,60: 4: 39,57: 5: 76$.

- consobrina $1: 25,34 ; 2: 63: 3: 8,23,28,32,41,49,60 ; 4$ : 39,$60 ; 5: 75 ; 18$ (suppl.): 41 .

- - var. sororia $3: 23,41 ; 5: 76$

- curtipes $3: 23,60 ; 4: 57: 5: 77$.

- cyanoxantha $3: 41: 5: 76: 6-7: 60,61,69,70,72,73 ; 8: 12$; 10: 17, 100:18: 3: 18(suppl.): 12 .

- f. pallida 6-7: 60 .

- decolorans $2: 63 ; 3: 23,28,32,41,49,60 ; 4: 39,58,60 ; 5$ : 76. 77,$79 ; 6-7: 37 ; 14: 51 ; 18$ (suppl.): 41, 108, 115, 117.

- delica $2: 63 ; 3: 22,28,31,41,49,60 ; 4: 39,57 ; 5: 75 ; 6-7$ : $61,70,72,73 ; 17: 66$

- var. delica 17:66.

- var. trachyspora 17:66

— densifolia $2: 63 ; 3: 23,31,41,49,60 ; 4: 57 ; 5: 75 ; 6-7: 27$; 17: 66 .

- depallens 3: 32: 4: 62:5: 78 .

- drimeia $1: 34 ; 3: 23 ; 5: 78$.

- elatior 2: 63 .

- emetica $1: 23,25,34,45 ; 2: 63 ; 3: 8,23,28,32,41,49,60$; $4: 40,58 ; 5: 76-78 ; 6-7: 60 ; 12: 55 ; 18$ (suppl.): 41 .

_ - var. crenulata $3: 67 ; 4: 40 ; 5: 77$.

- - var. silvestris f. phyllophila $4: 40: 5: 78 ; 6-7: 28$.

— — f. pithyophila $3: 60.67 ; 4: 40.58: 5: 78: 6-7: 28$.

- exalbicans $3: 41 ; 5: 78$.

- expallens 5: 78 .

- fallax 1: $34: 5: 78$.

— farinipes 6-7:27, 70;9:37, 38 .

- fellea $1: 25 ; 6-7: 27 ; 9: 37,38 ; 17: 66$.

- firmula $5: 78 ; 6-7: 28$.

- flava 2: 63: 18(suppl.): 45, 116, 117.

- foetens 1: 25. 34, 45:2: 13, 63; 3: 8, 23, 28, 31, 41, 49, 60; $4: 39,57,69 ; 5: 75 ; 6-7: 71 ; 8: 12 ; 9: 38 ; 17: 66$.

— fragilis $1: 25,34 ; 2: 63 ; 3: 23,28,32 ; 4: 40,58 ; 5: 78 ; 6-7$ : 28.

- - var. nivea $4: 58: 5: 78$

- fuscovinacea $3: 32 ; 4: 62: 5: 77$.

- gracilis 5: 78 .

- gracillima $3: 41 ; 5: 78$.

- graveolens $3: 23 ; 4: 39$.

- grisea $3: 41: 5: 76$.

— heterophylla $2: 63 ; 3: 23,60 ; 4: 57 ; 5: 76 ; 6-7: 60$. 
- $\mathrm{f}$. viridis $3: 23,28,41,49: 5: 75$

— integra $2: 63 ; 3: 8,23,32,41,49 ; 4: 40 ; 5: 77$.

- laricina var. ruberrima 5: 77 .

- lateritia 5: 77.

— laurocerasi 8: 12; 9: 38 .

— lepida 3: 23, 28, 49; 4: 57; 5: 76; 6-7:60;8:12.

- - var. lactea $3: 23,49 ; 5: 76$.

- lilacea $3: 49.60 ; 4: 57 ; 5: 76: 6-7: 27$.

- - var. carnicolor 5: 76 .

- lutea $2: 63 ; 3: 23,28,41,49,60 ; 4: 40,60 ; 5: 77 ; 6-7: 70: 8$ : 12.

— - var. flavida 5: 77; 6-7: 28 .

- luteotacta 5: 78: 17:66.

- maculata 5: 78; 17: 59, 66.

- mairei $1: 34 ; 2: 63 ; 3: 23 ; 4: 40,44,58,62 ; 5: 78 ; 6-7: 28$; 17: 66 .

- mariae 6-7: 27.

— melliolens 3: 32; 5: 77.

- mollis $2: 28 ; 3: 60: 5: 77$.

- nauseosa $3: 23: 4: 40,57 ; 5: 77 ; 6-7: 28$

- - var. flavida $5: 77 ; 6-7: 28$.

— nigricans $2: 63: 3: 23 ; 4: 39 ; 5: 75$.

— nitida $3: 41,49,67 ; 4: 39 ; 5: 77,78 ; 17: 66$.

- obscura $2: 63: 3: 23,49: 5: 76: 14: 49,51$.

— ochroleuca $1: 34 ; 2: 63: 3: 28,60,67: 5: 76 ; 6-7: 27,70 ; 9$ : 38.

- odorata 6-7: 28.

- olivacea $3: 23,32,60 ; 4: 40 ; 5: 76,77 ; 8: 12$.

- olivascens 5: 77 .

_ paludosa $2: 63: 3: 8,23,28,32,41,49,60: 4: 40,57 ; 5: 77$; 14: $51 ; 18$ (suppl.): 41, 97-99, 108, 115, 117.

— pectinata $3: 23,41 ; 5: 76 ; 6-7: 27,70 ; 9: 38$.

- pseudoaeruginea 5: 76 .

— pseudodelica $3: 23 ; 5: 76$.

- pseudointegra $3: 60 ; 5: 76: 6-7: 60,64,69,70,73: 8: 12 ; 18$ : 3.

- puellaris $3: 23,32,41,49,60 ; 4: 40,57 ; 5: 77,78 ; 6-7: 28$ : 17: 66 .

- pulchella 5: 78 .

- purpurata 3: 23; 4: 57 .

— queletii $1: 34 ; 2: 63 ; 3: 23,28,32,49.60 ; 4: 58 ; 5: 78$.

- rhodopoda 5: 5: 78 .

- romellii 3: 23, 28, 32, 41, 49, 60; 4: 40, 57; 5: 77.

— rosacea $2: 63: 3: 23,60 ; 5: 78$.

— rosea $4: 57: 5: 76$.

- roseipes $5: 77$.

- rubra 1: 25, 34

— sanquinea 1: $34,45: 2: 63 ; 3: 23,28,41,49 ; 4: 58 ; 5: 78$.

— - var. rosacea $3: 23,32,60 ; 4: 40 ; 5: 78 ; 6-7: 28$.

— sardonia $1: 25,34 ; 3: 23: 4: 40: 5: 78 ; 6-7: 28 ; 9: 37$.

— sororia 3: 23, 41, 49; 5: 76; 6-7: 70 .

— sphagnicola 4: 39: 5: 77; 7-8: 27.

- sphagnophila 6-7: 27.

- subfoetens 17: 59, 66.

- subrubens 4: 57.

- turcii 4: 40, 57:5: 76 .

- velenovskyi 3: 60:4: 40, 57: 5: 77

— venosa 3: 23, 32, 41, 49, 60; 4: 39, 40, 57: 5: 77.

_ - var. subingrata 4: 57: 5: 77.

— versicolor $3: 23 ; 4: 40,58 ; 5: 77,78 ; 6-7: 27,28$

- - var. intensior 6-7: 27.

- vesca $2: 63: 3: 23,28,31,41,49,60 ; 4: 39,60 ; 5: 77 ; 6-7$ : 70. 72; 18 (suppl.): $41,116,117$.

- veternosa 1: 34

—vinosa $2: 63 ; 3: 23,28.32,41,49.60 ; 4: 39,57: 5: 77 ; 8: 39$; 18(suppl.): $116,117$.

— violacea $3: 60,67 ; 4: 40 ; 5: 78$

— violeipes 6-7: 27, 70 .
— vitellina 5: 77 .

- xerampelina $2: 63 ; 3: 9,23,28,32,41,49,60 ; 4: 57 ; 5: 76$; 6-7: 27; 17: 67; 18(suppl.): 41, 116, 117 .

_ - var. barlae 3: 23, 32, 49; 5: 76 .

_ - var. elaeodes 5: 76; 6-7: 27.

- - var. erythropus $3: 49,60,67 ; 5: 76$.

- - var. fusca $3: 41,60 ; 5: 76$.

— - var. graveolens $3: 60 ; 4: 57 ; 5: 76 ; 6-7: 27$.

_ - - f. subrubens 4: 39 .

_ - var. graveolens-subrubens 3: 23; 5: 76.

_ - var. olivascens $3: 49,60 ; 5: 76 ; 6-7: 27$.

— - var. pascua 3: 60; 5: 76 .

- - var. pseudomelliolens 3: 60: 5: 76; 6-7: 27.

- - var. f. purpurata 5: 76 .

- zonatula $3: 60 ; 5 ; 78 ; 17: 66$

Sabouraudites gypseum 1: 49 .

Sarcodon 1 : $35 ; 3: 24 ; 5: 87 ; 6-7: 30$

- fennicus 5: 87; 6-7: 30 .

— fragilis $3: 42 ; 5: 87$.

— imbricatus $3: 24,29,42,51: 4: 59,60 ; 5: 87 ; 6-7: 30 ; 8: 37$, 47, 48, 59; 18(suppl.): 6-7, 41, 116, 117.

— infundibulus $3: 51 ; 5: 87 ; 6-7: 30$.

- laevigatus 6-7: 30 .

- scabrosus 3: 24.

- - var. fennicus 5: 87 .

- subsquamosus 3: 24. 51; 4: 59: 5: 87: 6-7: 30.

— violascens 4: 59:5:87.

Sarcoscypha 3: 52; $5: 97 ; 13: 51 ; 14: 125$.

- coccinea 10: 79 .

— melastoma $3: 43 ; 5: 97$.

- protracta 4: 27; 6-7: 105 .

Sarcosoma 14: 125.

Sarcosphaera eximia 1: 36 .

Schizophyllum 13: 37 .

- alneum 2: 63.

- commune $2: 63 ; 6-7: 10 ; 11: 25 ; 16: 3 ; 17: 34 ; 18: 35$

Schizostoma 17: 59.

- lacerata 17: 71

Scirrhia agrostidis 15: 41, 47 .

Scleroderma 1: 35,$45 ; 5: 93 ; 10: 170.175 ; 18$ (suppl.): 6 .

— aurantium 1: 25; 5: 93; 6-7: 67, 72; 18(suppl.): 6 .

- - var. spadiceum 5: 93: 6-7: 32, 67.

- bovista 2: 50; 3: 67: 5: 93; 6-7:32, 111, 112; 10: 175.

— cepa 3: 50: 5: 93.

- flaccidum 10: 175.

— verrucosum 1: $25 ; 3: 50,67 ; 5: 93 ; 6-7: 67 ; 10: 174$.

— vulgare 5: 93; 6-7: 67: 10: 175 .

Scleroderris lagerbergii 14: 29.

Sclerotinia 20: 30 .

— pirolae 20: 28-31.

- sclerotiorum 16: 3; 20: 3 .

— tuberosa 3: 30, 32, 33, 53; 4: 28; 5: 96.

Scolecotrichum graminis 13: 9, 10, 13, 14.

Scopulariopsis 1: 56,58 .

Scutellinia 14: 125 .

Scutiger ovinus $8: 19$.

Sepedonium 18(suppl.): 74.

Septogloeum 15: 59.

- agrostidis 15: 59.

- oxysporum 15: 56, 57, 59-62.

Septoria gregaria 17: 1, 15, 16.

- oxyspora 17: 3.7.16.

- stellariae 17: 16

Sepultaria 9: 5,7 .

- arenicola 9: 8 .

- arenosa 9: 7,8.

Serpula 5: 86 .

— rufa var. pinicola 5: 92. 
- serpens var. pinicola 5: 92 .

Singerella 14: 113, 114; 15: 17; 18: 49.

- clitocyboides 15: 16, 17.

- hydrogramma 14: 113, 114; 15: 16, 17.

Singeriella 15: 17.

Sistotrema confluens 5: 87 .

- fagineum 5: 86.

- sublamellosum 5: 87.

Sordaria fimicola 12: 55

Sorosporium 17: 5.

- saponariae 17: 6 .

Sowerbyella 14: 125, 139

Sparassis crispa 5: 82; 6-7: 28

Spathularia 9: 24.

- clavata $3: 30,43,52 ; 4: 59 ; 5: 95 ; 6-7: 71$.

Spermospora 13: 9, 10, 27.

— ciliata 13: 9-12.

- subulata 13: 9 .

— - f. ciliata 13:9

Sphacelia typhina 15: 49 .

Sphaerella gangraena 15: 56, 59.

Sphaeria artocreas 15: 44.

- gangraena 15: 56.

Sphaerotheca alchemillae 8: 6 .

- epilobii 4: 22.

- euphorbiae 6-7: 98-100.

— fugax $4: 22 ; 8: 6$.

— fuliginea $4: 22 ; 6-7: 100 ; 8: 5,6$.

- fusca 4: 23 .

- humuli 4: 23 .

- macularis 4: 21, 23:6-7: 100 .

- melampyri 8: 6 .

- mors-uvae 4: 24; 6-7: 99.

- pannosa 4: 24; 6-7: 100 .

- volkartii 4: 24

Spicaria 1: 84 .

Spongipellis borealis $3: 29: 5: 89 ; 8: 14 ; 11: 23-26$.

_ - var. spathularius 3: 29, 51; 5: 89 .

— fissilis 6-7: 31

- spumeus 3: 51; 5: 89; 6-7: 31; 8: 19.

Sporobolomyces salmonicolor 12: 57.

Sporocybe resinae 14: 135

Sporotrichum 1: 184

Spumaria alba 19: 12, 13.

- physaroides 19: 15

Staphylococcus aureus 18(suppl.): 6 .

- pyogenes var. aureus 18(suppl.): 6 .

Stemonitis 14: 65, 67; 17: 31 .

— axifera 14: 66; 19: 9, 13 .

- elegantula 19: 12, 13, 15 .

- fasciculata 19: 8, 13 .

— ferruginea 14: 66, 79; 19: 13.

_ - var. smithii 14: 66; 19: 13 .

- favogenita 14: 66, 79; 19: 13.

- friesiana 19: 10, 13.

— fusca 14: 65; 19: 8, 9, 13.

_ - var. confluens 14: $65 ; 19: 6$

_ - var. flaccida 14: 65; 19: 6 .

_ - var. nigricans 14: 65

- - var. rufescens 14: 65

- - var. trechinospora 14: 65

- herbatica 19: 1, 6, 13 .

- ovata 19: 10,13.

- pallida 17: 19, 24; 19: 13 .

- physaroides 19: 12, 13.

-- smithii 14: 66; 19: 13 .

- splendens 14: 65; 19: 13 .

_ - var. flaccida 14: 65, 66:19: 13 .
— typhoides 19: 13

— virginiensis 19: 1, 6, 13 .

Stemonitopsis dictyospora 19: 1, 6, 13.

- typhina 19: 10, 13.

Stereocaulon 6-7: 112.

Stereum 5: 86, 88; 6-7: 29, 30; 12: 50 .

- bicolor 6-7: 29 .

— fasciatum 6-7: 29

- fuscum 6-7: 29 .

- gausapatum 6-7: 30; 17: 33, 34 .

- hirsutum 3: 23, 29, 32, 42, 51, 61: 4: 40, 59; 5: 86; 10: 181; 12: $48-50 ; 17: 34$.

— insignitum 17: 33,34

- pini 5: 86

- purpureum 3: 29, 32, 42, 61; 4: 40, 59; 5: 86; 10: 181; 12: 47-50; 13: 45.

— rugosum $3: 23,29,51 ; 4: 40,59 ; 5: 86 ; 6-7: 42$.

— sanguinolentum $3: 29,61 ; 4: 59 ; 5: 86 ; 10: 178,179,182$; $11: 29,31 ; 12: 47-51 ; 13: 45$.

- spadiceum 6-7: 30 .

Stevensonia $3: 24,43 ; 5: 97$.

Stomiopeltis antarctica 17: 14.

Streptomyces 18(suppl.): 74 .

- thermovulgaris 18(suppl.): 74.

Strobilurus 14: 50, 51 .

- stephanocystis 17: 68

Stromatinia 20: 30 .

- pirolae 20: 28 .

Stropharia 1: $18,31,36,43,46 ; 2: 48: 3: 14 ; 10: 115 ; 14: 114$; 18(suppl.): $53-55$

- aeruginosa $1: 25,31,43 ; 2: 31,32,63 ; 3: 8,13,28,40,49$, $67 ; 4: 38,55 ; 5: 67 ; 6-7: 70: 10: 180 ; 18$ (suppl.): 41 .

— albocyanea $3: 67 ; 4: 55 ; 5: 67$.

- albonitens $2: 31: 3: 8,13$.

- coronilla $1: 31,43 ; 3: 22,28,40,49 ; 5: 67$

- cyanea 2: 31,63 .

- depilata 1: $25,31,43 ; 2: 63 ; 3: 8,9,13,16,28,40,49 ; 4: 55$ 5: 67,103 .

- hornemannii 2: 63; 3: 28: 5: 67: 6-7: 25; 10: 180: 18(suppl.): 41 .

- luteonitens 3: 22; 5: 67

- melasperma 3: 22, 28, 40, 49; 4: 55; 5: 64, 67.

- merdaria 2: 63 .

- rugosoannulata 18 (suppl.): $10,17,53,55,75,100$

— semiglobata $2: 63 ; 3: 22,28.40,49,59 ; 4: 38,55 ; 5: 67: 10$ : $114,180$.

- - f. etiolata 4: 55: 5: 67

- - var. stercoraria 4: 55

- separata 2: 63 .

- spintrigera $4: 28,39 ; 5: 71$

— squamosa $3: 59 ; 5: 67: 6-7: 70$

- stercoraria 3:22, 28, 31, 40, 59:4:38:5:67

- - var. microspora 3: 40; 5: 67 .

Stysanus resinae 14: 135 .

Suillus $2: 63 ; 6-7: 6 ; 10: 170,171,178 ; 18: 28: 18$ (suppl.): 103 , 116,117

- aeruginascens 5: 8

- amabilis 10: 174

- bellinii 10: 170

- bovinus $2: 53 ; 3: 19,25,43,54 ; 4: 29,45: 5: 8: 9: 39,41,42$; 10: 173, 180:18: 7; 18(suppl.): 6, 115, 117 .

- brevipes 10: 171, 173.

- cyanescens 6-7: 58 .

- elegans $2: 53 ; 3: 25: 4: 29 ; 5: 8: 6-7: 6: 9: 39 ; 10: 180$ 18( (suppl.): 6

- flavidus $2: 53: 3: 19,43: 4: 45 ; 5: 8: 18$ (suppl.): 6 .

— granulatus $2: 53: 3: 19,25,30,33,43,54: 5: 8: 6-7: 53,54$ $68 ; 8: 11: 9: 36: 10: 170,173 ; 18: 4.7 ; 18$ (suppl.): 6,34 . 
- grevillei 2: 42, 53; 5: 8; 6-7: 6; 9: 39; 41, 42; 10: 169-171, 173; 18 (suppl.): 41 .

- lakei 10: 171, 174.

- lapponicus 18: 27, 28.

- luteus $2: 53 ; 3: 19,25,30.33,43,54 ; 4: 29,45 ; 5: 8 ; 6-7: 37$, 74; $9: 39,41,42,46 ; 10: 170,174 ; 14: 51 ; 18: 7 ; 18$ (suppl.): $6-7,35-37,41,87,89-91,108,115,117$.

- piperatus $2: 53 ; 3: 19,25,30,33,43,54 ; 4: 29,45 ; 5: 8 ; 10$ : $174 ; 18: 12$.

_ variegatus $2: 53 ; 3: 19,25,30,33,43,54 ; 4: 39,41,42 ; 5: 8$; 9: $39,41,42 ; 10: 170,180,182 ; 14: 49-52 ; 18: 27,28$; 18(suppl.): 6, 41, 97-99, 108, 115, 117.

- viscidus 5: 8 .

Symphytocarpus amaurochaetoides 19: 1, 6, 7, 10, 13, 16 .

_ flaccidus 19: 13 .

Taphrina entomospora 17: 14.

Tarzetta 14: 105, 106, 116, 117, 119, 125, 138-142; 17: 75.

- catinus 13: $55 ; 14: 116-119,139-141$.

- cupularis 14: 138 .

- pusilla 14: 116-119, 138, 139; 15: 27.

- spurcata 14: 116-120, 138, 139, 141.

Telamonia $2: 64 ; 4: 51,60 ; 5: 49,51,55 ; 6-7: 20,24$.

Telimenella 15: 56

- gangraena 15: 47, 56-59, 61, 62.

Tephrocybe 14: 50, 51; 19: 51 .

- palustris 14: 49, 52 .

Tetracladium maxilliformis 13: 16, 18, 20, 21 .

Thamnolia vermicularis 10: 42 .

Thelephora contorta 6-7: 30 .

Thermomonospora 18(suppl.): 74.

Tilletia calamagrostidis 6-7: 88 .

- corcontica 6-7: 88.

Tilmadoche gracilenta 19: 12, 13.

- mutabilis 19: 13.

- nutans 19: 12, 13.

- - var. rigida 19: $12,13,16$

Titea maxilliformis 13: 21 .

Tolyposporium 17: 5 .

Tomentella 5: 88 .

- microspora 5: 88 .

- mollis 6-7: 30 .

Topospora 14: 35-37, 39-41, 44.

Torula 1: 56,57 .

Torulopsis glabrata 15: 9 .

Trachypus 2: 26.

- leucophaeus 2: 26.

Trametes 5: 91, 92; 18: 43 .

- abietis $3: 53 ; 5: 92$

— cinnabarina $3: 8,24,32,42,51,61 ; 4: 41 ; 5: 86,91 ; 6-7: 31$; 16: 3 .

- fibrillosa 20: 8 .

- gibbosa 17: 38 .

- heteromorpha 3: 32, 61; 5: 91.

- hirsuta 17: 38

— mollis 3: 29, 32; 4: 41; 5: 91; 8: 15 .

- odorata $3: 24,29,32,42,51,61 ; 5: 91 ; 8: 15$

— pini 3: 53; 5: 92; 8: 18 .

- quercina 6-7: 42 .

_ - f. lenzitoidea 17: 37.

_ rubescens $3: 68 ; 4: 41 ; 5: 91 ; 8: 16$.

- salicina 18: $43,47,48$.

- - var. greschikii 18: 43, 47

— serialis $1: 61,68-71 ; 3: 24 ; 4: 44,59 ; 5: 91 ; 8: 15 ; 10: 182$

_ - var. resupinata $5: 91 ; 6-7: 31$.

- serpens 18: 48

— squalens $3: 51 ; 5: 91$.

- suaveolens 10: 181 .

— trogii 18: 47 .
- unicolor 6-7: 42

- velutina 17: 38 .

- versicolor 17: 38

Tremella encephala $3: 29,42 ; 4: 58 ; 5: 81$.

- fimbriata $3: 61 ; 5: 81$.

— foliacea $2: 49 ; 3: 29,50 ; 5: 81 ; 6-7: 31$.

- lutescens 5: 81 .

- mesenterica 4: 43; $5: 81$.

Tremellodon gelatinosus(-um) $5: 82$.

Tricellula aquatica 13: 16, 18-20.

Trichaptum biforme(-is) 17: 37.

Trichia 14: 72, 73, 75, 79, 80; 19: 14, 15

— affinis 14: 73; 17: 13 .

— anomala 19: 10, 13, 14.

— botrytis 6-7: 110; 14: 54, 74, 75; 17: 24; 19: 13, 16.

— - var. lateritia 14: 75; 19: 13 .

- chrysosperma 19: 13.

- clavata 19: 11, 13.

- contorta 14: 74; 19: 13, 15.

- - var. inconspicua 14: 74; 19: 13.

- - var. karstenii 19: 15.

- decipiens 6-7: 110; 14: 74, 79; 17: 31; 19: 9, 13.

— - var. sessile 14: 74, 80 .

- fallax 19: 13 .

— favoginea $6-7: 110 ; 14: 72 ; 17: 31 ; 19: 13,16$.

- floriformis 14: 75, 78; 19: 13 .

— fragilis $14: 54 ; 19: 13$.

- lateritia 14: 75 .

- lutescens 14: 74, 78; 19: 13

- nigripes 19: 13.

- nutans 19: 14.

— persimilis 14: 72, 79, 80; 19: 13, 16 .

— proximella 19: 13, 16 .

- purpurascens 14: 54; 19: 13, 16.

— pusilla 14: 74; 19: 13 .

- pyriformis 19: 12, 13 .

- scabra 14: 73, 79; 19: 13, 14.

- subfusca 14: 75; 19: 13.

- turbinata 19: 13 .

— varia 14: 74, 80; 17: 31; 19: 9, 13.

- - var. olivacea 19: 13 .

Trichoderma 6-7: 14; 14: 20; 18(suppl.): 86.

- lignorum 14: 5 .

— viride 11: 25,$26 ; 12: 61 ; 14: 5 ; 18$ (suppl.): 51 .

Tricholoma 1: 16, 26, 28, 36, 41, 46; 3: 10; 4: 60; 5: 36; 10: 11 , $12,169,171 ; 11: 39 ; 14: 85,86,88-90,132 ; 15: 23 ; 18: 50$; 18(suppl.): 34, 82, 103, 114; 19: 26.

— aestuans 14: 51 .

— albobrunneum 1: 25, 29, 42; 2: 64; 3: 34, 63; 5: 19; 15: 23.

— album $1: 25,28,41 ; 2: 49,64 ; 3: 8,26,34,45,55 ; 4: 47,60$; 5: $19 ; 6$-7: 44, 46, 63, 71; 15: 23; 18(suppl.): 41 .

- aromaticum 15: 16.

— aurantium 2: 63; 5: 19; 15: 23.

— bufonium 15: 23; 18: 29.

— caligatum 14: 88; 15: 23, 27.

- cingulatum 5: 20 .

- columbetta $1: 29,41 ; 2: 64 ; 3: 26 ; 5: 19 ; 6-7: 44,69,73 ; 8$ : 10; 18: 3 .

- cuneifolium 5: 19; 6-7: 9 .

- decorum 2: 64; 3: 8 .

- equestre 1: 88; $2: 64 ; 3: 8,10,20,26,45 ; 4: 42,47,60 ; 5$ : $19 ; 18$ (suppl.): 87-92, 102-105, 120-123.

- flavobrunneum $2: 64 ; 3: 20,26,30,34,45,55 ; 4: 31,46$, $60 ; 5: 19 ; 9: 40,41 ; 15: 23$.

- flavovirens $2: 64 ; 5: 19 ; 6-7: 37 ; 8: 43,46,48,49,51,59$, 60; 14: 51; 15: 23; 18(suppl.): $6,7,39,41,81,83,87,108$, 115,117 .

— focale $3: 20 ; 5: 19$. 
- fucatum 3: 55, 63; 5: 19; 6-7: 9

— gambosum 3: 20; 5: 12; 6-7: 54 .

- geminum 5: 29.

- georgii 18(suppl.): 7

— imbricatum 2: 64; 3: 20, 26, 30, 34, 45; 4: 31, 60; 5: 19; 9: 40, 43; 15: 23.

— inamoenum 1: 29, 41; 2: 64; 3: 8, 20, 26, 30, 34, 45, 55; 4: 42,$47 ; 5: 19 ; 6-7: 9 ; 14: 50,88$.

— inodermeum var. amarum 4: 47; 5: 19.

- interveniens 10: 114.

— irinum 6-7: 9, 46, 69; 14: 129, 132.

- lascivum 4: 47; 5: 19; 6-7: 9, 46, 72, 73; 8: 10.

- macrocephalum 17: 68 .

- melaleucum 2: 64 .

— myomyces $3: 20 ; 4: 60 ; 5: 20$.

- nudum $1: 85 ; 2: 64 ; 3: 8,10 ; 16: 12,13 ; 18:($ suppl.): 26,27 , 34.

— panaeolum 11: 39 .

- - f. caespitosum 11: 39.

- pardinum 1: 28, 29, 41 .

- personatum 2: 64 .

— pessundatum 1: $25,29,41 ; 2: 64 ; 3: 8,52,63 ; 4: 46 ; 5: 19$; 6-7: 38 .

— portentosum $1: 37,88 ; 2: 64 ; 3: 26,34,63 ; 4: 47,60 ; 5: 19$; 18(suppl.): 29, 30, 81 .

- - var. leucoxanthum 5: 19.

— psammopus 3: 26; 5: 19; 10: 169.

- pubifolium 1: 29, 41 .

- putidum 3: 25.

- quinquepartitum 3: 20; 5: 19.

- resplendens $3: 26 ; 5: 19$.

— rutilans $2: 49,64 ; 3: 8,9,30 ; 9: 40$

- saponaceum 1: $29,42,88 ; 2: 64 ; 3: 8,20,26,36,45 ; 4: 60$; 5: 19, 20, 41; 6-7: 9, 10, 70; 14: 88, 89; 15: 23

_- var. ardosiacum 3: 20, 34, 55; 5: 20; 6-7: 9, 46.

_ - var. atrovirens 5: 20; 6-7: 9 .

_ - var. napipes $3: 34 ; 5: 20 ; 6-7: 9$.

_ - var. squamosum 5: 20 .

— scalpturatum 3: 20; 5: 20 .

- sciodes 5: 20.

— sejunctum 2: 64; 3: 20, 55, 63; 5: 19; 14: 88.

- sordidum 2: 64 .

— striatum 1: 29, 42; 2: 64; 3: 34; 5: 19.

- sudum 6-7: 9.

— sulphureum 1: 25, 29, 42; 2: 64; 3: 63; 5: 19; 6-7: 44-46, 63, 69, 70, 72, 73; 8: 10; 14: 88; 18: 19 .

— terreum 1: 88; 3: 20, 26; 4: 60; 5: 20; 6-7: 71 .

- - var. triste 3: 52 .

- tigrinum 1: 28, 29, 42 .

- tridentinum 3: 63; 5: 19 .

— tumidum 5: 20.

- ulmarium 2: 64.

— ustale 5: 19; 6-7: 9 .

— vaccinum $2: 64 ; 3: 8,26,34,45,63 ; 4: 60 ; 5: 19$.

- verrucipes 10: 115 .

— virgatum 1: $25,29,42 ; 2: 64 ; 3: 8,10,20,26,34,45,55 ; 4$ : $60 ; 5: 20 ; 18$ (suppl.): 41 .

— viridilutescens $3: 55,63 ; 5: 19$.

Tricholomopsis 2: 54, 64 .

— decora $2: 64 ; 3: 44,54,63 ; 4: 30,46 ; 5: 15 ; 6-7: 8 ; 14: 50$.

—ornata 4: 30,$46 ; 5: 15$.

— platyphylla $2: 64 ; 3: 30,33,44,54 ; 4: 30$.

- rutilans $2: 64 ; 3: 20,26,30,33,44,54 ; 4: 30,46 ; 5: 15 ; 9$ : 40, 41; 14: 50 .

- - var. variegatus 6-7: 8

Trichophyton 1: 50,84 .

- album 1: 84.

- crateriforme 1: 48
- equinum 1: 48

- faviforme album 1: 48 .

- - discoides 1: 48

- gypseum 1: 50 .

- - asteroides 1: $48,51$.

- - granulosum 1: 48, 49 .

- - persicolor 1: 48 .

— interdigitale 1: 49, 51-53, 55, 58.

— rosaceum 1: 48 .

- sulphureum 1: 49 .

- violaceum 1: 48, 50-52, 55

Trichoscyphella hahniana 11: 41 .

- willkommii 11: 41 .

Trichosporon 15: 10 .

Trichothecium 12: 53, 54, 57.

— roseum 12: 53, 54, 56, 57; 18(suppl.): 51.

Tubaria 1: 31; 4: 38, 51; 5: 48; 17: 64; 18: 55, 56.

- confragosa 18: 55 .

- conspersa $3: 46,56 ; 4: 34,49 ; 5: 40 ; 6-7: 15$.

- ferruginea $4: 34,49 ; 5: 40$.

— furfuracea $2: 64 ; 3: 27,35,46,56 ; 5: 40 ; 18: 55,56$

— minima 3: 56; 5: 40 .

- minutalis $3: 56 ; 4: 49 ; 5: 40$.

- pallidispora 3: 46, 56, 65; 4: 28, 34, 42, 49; 5: 40.

_ pellucida $3: 56,65 ; 4: 28,34,49 ; 5: 40$.

- - f. segestria 5: 40 .

— phaeophylla $3: 35 ; 5: 40$.

— praestans 4: 49; 5: 40 .

- pseudoconspersa 5: 40 .

- scolecina 4: 35 .

— trigonophylla $4: 34 ; 5: 40 ; 17: 64$.

- umbrina 4: 35 .

Tubercularia communis 5: 98 .

Tubifera 14: 79 .

- ferruginosa 6-7: 110; 14: 71; 19: 12-14.

Tubiporus luridus 18: 9 .

Tubulina cylindrica 19: 14 .

Tuburcinia carcinoides 20: 16, 17.

Tulostoma brumale 6-7: 111 .

Tylopilus 2: 64 .

- felleus $2: 53 ; 3: 19,25,30,33,43,54 ; 4: 29,60 ; 5: 8 ; 16: 3$; 18: $28 ; 18$ (suppl.): 41 .

Typhula 5: 84 .

- phacorrhiza 5: 84 .

- trifolii 13: 21.

Tyromyces 13: 6 .

- albidus 8: 19.

- aneirinus 8: 19

- caesius 8: 19.

- cinerascens 8: 19; 10: 182.

— erubescens 8: 19 .

- fissilis 10: 179, 181.

- floriformis 8: 19 .

- fragilis 8: 19; 10: 182.

- lacteus 8: 19 .

- lowei 20: 2.

- ptychogaster 8: 19.

- semipileatus 8: 20 .

- sericeomollis 8: 20.

- tephroleucus 8: 20.

- trabeus 8: 20.

Uncinula nothofagi 17: 14 .

- prunastri 4: 24

- salicis 4: 24; 6-7: 100

- tulasnei 4: 24; 5: 98.

Underwoodia 14: 102, 103.

- beatonii 14: 103, 104.

Ungulina 5: 91. 
— annosa 3: 24:5:91.

- benzoina 5: 91; 6-7: 31 .

— betulina 3: 24, 29, 32, 42, 51, 61; 4: 41, 59; 5: 91.

- corrugis 17: 38

- fomentaria $2: 49 ; 3: 24,29,32,42,51,61 ; 4: 41,59 ; 5: 91$.

— fuliginosa $3: 24,42 ; 5: 91$.

- marginata 3: 24, 29, 32, 42, 51, 61; 4: 41, 59; 5: 91 .

- resinosa 3: 24, 29, 42, 61; 5: 91 .

Uredo chascolytri 17: 12

- chiliotrichi 17: 13 .

- festucae 8: 7 .

Urnula 14: 125; 17: 41.

Urocystis 17: 6 .

- agropyri 17: 6 .

- alopecuri 17: 6 .

- avenastri 17: 6 .

- carcinoides 20: 16-18.

- ferrarisiana 20: 16-18.

- melicae 17: 6 .

- occulta 17: 6 .

- permagna 17: 1, 6 .

- poae 17: 6

- tessellata 17: 3,6

- ulei 17: 3,6.

Uromyces airae-flexuosae 8: 7

- armeriae 17: 9, 10.

- armeriicola 17: 10 .

- commelinae 17: 11

— ellipticus 17: 12.

- fallens $4: 18 ; 8: 5$.

- guayacuru 17: 12.

- junci 17: 11, 14

- lapponicus 6-7: 97; 8: 7

- limonii 17: 11, 12.

- lineolatus 6-7: 97.

- mulini 17: 12 .

— nordenskjoeldii 17: 12.

- patagonicus 17: 12 .

- peckianus 17: 3, 12.

— pisi 4: 18.

- plantaginis 17: 12 .

- polygoni-aviculariae 8: 7; 17: 12 .

- pratiae 17: 12

- primaverilis 17: 12.

- quinchamalii 17: 12.

- scirpi 6-7: 97.

— sommerfeltii 8: 5 .

- striatus 17: 12.

- symphyostemi 17: 12.

- viciae-fabae 6-7: 97.

Ustilago 6-7: 88, 91

- bromivora 17: 6

— bullata 17: 6 .

- calamagrostidis 6-7: 88-93.

- corcontica 6-7: 88-93.

- cortaderiae 17: 6 .

- hypodytes 17: 6 .

- levis 17: 6

- scrobiculata 6-7: 88-93.

- striiformis 17: 6 .

Ustulina vulgaris 5: 89 .

Valsa sorbi 5: 98

Varicosporium elodeae 13: 16, 18, 20, 21.

Vascellum pratense 17: 70 .

Verpa 14: 124.
Verticillium 18(suppl.): 10, 51.

- malthousei 18(suppl.): 19, 85 .

Vibrissea truncorum 3: 32, 42, 62; 4: 41; 5: 95.

Volvaria 1: 18, 30, 36, 42, 46; $2: 64$.

- speciosa 1: 25; 4: 33, 42 .

Volvariella 2: 64; 3: 64; 18(suppl.): 54, 59, 75.

— plumulosa 3: 21; 5: 35 .

_ pusilla $3: 27 ; 5: 35$

— - var. biloba 3: $35 ; 5: 35$

- speciosa 2: 64; $3: 46,56,64 ; 5: 35$.

- volvacea 18(suppl.): 53 .

Volucrispora 13: 17 .

- graminea 13: 16-20.

Vuilleminia comedens 5 : 85 .

Wojnowicia 19: 54 .

- graminis 19: 54-57.

Wynnella 14: 102 .

- silvicola 14: 102, 103; 17: 46.

Xanthochrous abietis 3: 53; 5: 92.

- cinnamomeus 3: 68; 4: 41; 5: 92.

- circinatus 5: 92; 6-7:31.

— - var. triqueter 5: 92 .

- dryophilus 4: 61; 5: 92.

- glomeratus 5: 92; 6-7: 31 .

- nodulosus $4: 43 ; 5: 92$

- obliquus 4: 44; 5: 92.

- perennis $3: 24,30,42,51,61 ; 4: 44,59 ; 5: 92$.

- pini 3: 53; 4: 59; 5: 92 .

— radiatus $3: 51 ; 4: 41,43,44 ; 5: 92 ; 6-7: 31$.

- rheades 4: 61; 5: 92 .

- tomentosus 5: 92 .

— vulpinus 4: 61; 5: 92.

Xerocomus 2: 64; 18(suppl.): 102, 103

— badius 2: 53; 3: 19, 25, 43, 54; 4: 29, 45; 5: 8; 6-7: 55 . 68,$70 ; 18$ : 3; 18(suppl.): $6,87-91,102-105,120-123$.

— chrysenteron $2: 53 ; 3: 43,54 ; 4: 45 ; 5: 8 ; 6-7: 46,53-56$, $59,60,68,70,73 ; 8: 11,23 ; 9: 36 ; 18: 5 ; 18$ (suppl.): 6

- coniferarum 18: 15 .

- impolitus 18: 10

- lanatus 18: 16

- parasiticus 6-7: 72

- porosporus 18: 13 .

- rubellus $5: 8$.

- subtomentosus 2: 53; 3: 19, 25, 33, 43, 54; 4: 29, 44; 5: $8 ; 6-7: 37,54,70 ; 18: 15 ; 18$ (suppl.): 6 .

- versicolor $3: 54 ; 4: 29 ; 5: 8 ; 6-7: 70$.

Xeromphalina 4: $5,47,62 ; 5: 25 ; 19: 53$.

- amara 14: 50

- campanella $2: 64 ; 3: 20,26,34,45,55 ; 4: 5-8,31,47$, $60 ; 5: 25,28 ; 10: 178,182 ; 11: 29,31 ; 12: 47-49,51 ; 13$ 45

— - var. (f.) badipes(-us) 3: 52; 4: 8; 5: 25 .

- - var. myriadea 4: 7 .

- caulicinalis (cauticinalis) 2: 64; 4: 5, 6, 8, 47; 5: 25; 6-7: 71.

- cornui $4: 5,8$.

- fraxinophila 4: 8

- fulvobulbillosa $2: 64 ; 5: 25$.

- kauffmaniana 4: 8 .

— picta 19: 52, 53 .

Xerula longipes 6-7: 46, 47, 73.

Xylaria hypoxylon $8: 9$.

- polymorpha 8: 9 .

Xylodon versiporus 5: 93 . 